\title{
Spatially resolved spectroscopy and chemical history of star-forming galaxies in the Hercules cluster: the effects of the environment
}

\author{
Petropoulou V. ${ }^{1}$, Vílchez J. ${ }^{1}$, Iglesias-Páramo J. ${ }^{1,2}$, Papaderos P. ${ }^{3}$, Magrini L. ${ }^{4}$, Cedrés \\ B. ${ }^{1,5}$, Reverte D. ${ }^{6}$ \\ ${ }^{1}$ Instituto de Astrofísica de Andalucía- C.S.I.C., Glorieta de la Astronomía, 18008 Granada, Spain; \\ ${ }^{2}$ Centro Astronómico Hispano Alemán, C/ Jesús Durbán Remón 2-2, 04004 Almería, Spain; \\ ${ }^{3}$ Centro de Astrofísica da Universidade do Porto, Rua das Estrelas, 4150-762 Porto, Portugal; \\ ${ }^{4}$ INAF - Osservatorio Astrofisico di Arcetri, Largo E. Fermi 5, 50125 Firenze, Italy; \\ ${ }^{5}$ Instituto de Astrofísica de Canarias, C/ Vía Láctea s/n, 38205 La Laguna (Tenerife), Spain; \\ ${ }^{6}$ GRANTECAN S.A., Centro de Astrofísica de La Palma, C/ Cuesta de San José s/n, 38712 Breña Baja \\ (La Palma), Spain
}

\begin{abstract}
Spatially resolved spectroscopy has been obtained for a sample of 27 star-forming (SF) galaxies selected from our deep $\mathrm{H} \alpha$ survey of the Hercules cluster. We have applied spectral synthesis models to all emission-line spectra of this sample using the population synthesis code STARLIGHT and we have obtained fundamental parameters of the stellar components, as the mean metallicity and age. The emission-line spectra were corrected for underlying stellar absorption using these spectral synthesis models. Line fluxes were measured and $\mathrm{O} / \mathrm{H}$ and $\mathrm{N} / \mathrm{O}$ gas chemical abundances were obtained using the latest empirical calibrations. We have derived masses and total luminosities of the galaxies using available SDSS broadband photometry. The effects of cluster environment on the chemical evolution of galaxies and on their mass-metallicity (MZ) and luminosity-metallicity (LZ) relations were studied combining the derived gas metallicities, the mean stellar metallicities and ages, the masses and luminosities of galaxies and their existing HI data. We have found that our Hercules SF galaxies divide into three main subgroups: a) chemically evolved spirals with truncated ionized-gas disks and nearly flat oxygen gradients, witnessing the effect of ram-pressure stripping, b) chemically evolved dwarfs/irregulars populating the highest local densities, possible products of tidal interactions in preprocessing events, or c) less metallic dwarf galaxies which appear to be "newcomers" to the cluster, experiencing pressuretriggered star-formation. Most Hercules SF galaxies follow well defined MZ and LZ sequences (for both $\mathrm{O} / \mathrm{H}$ and $\mathrm{N} / \mathrm{O}$ ); though the dwarf/irregular galaxies located at the densest regions appear to be outliers to these global relations, suggesting a physical reason for the dispersion in these fundamental relations. The Hercules cluster appears to be currently assembling via the merger of smaller substructures, providing an ideal laboratory where the local environment has been found to be a key parameter to understand the chemical history of galaxies.
\end{abstract}

Subject headings: galaxy clusters: general — galaxy clusters: individual(Abel2151)

\section{INTRODUCTION}

The star formation history (SFH), gas-content and the mass exchange with the environment (infall of metal-poor gas and/or outflow of enriched material) are fundamental variables that regulate the chemical evolution of a galaxy. Clusters of galaxies host large numbers of galaxies of various sizes, luminosities and morphologies as well as a 
large mass of gas confined within a given volume of space. Thus galaxy clusters can provide an excellent place to study the impact of the environment on the aforementioned fundamental variables regulating the metal content of a galaxy.

The relation between stellar mass and metallicity is fundamental to constrain galaxy evolution scenarios in dense environments. From the observational point of view, Lequeux et al. (1979) was the first to point out that metallicity is strongly correlated with galaxy stellar mass or luminosity, with the more massive galaxies being metal richer. Since then a lot of work has been done, extending these correlations to large samples of galaxies (Skillman et al. 1989; Zaritsky et al. 1994; Lamareille et al. 2004, among others). This holds at all redshifts, although for a given stellar mass, more distant galaxies appear to have a lower metal content than local galaxies (e.g Savaglio et al. 2005; Erb et al. 2006). Maiolino et al. (2008) argue that both the zero point and the slope of the MZ relation are a function of redshift.

Many different scenarios have been proposed for the physical mechanisms underlying such a relation. According to Finlator \& Davé (2008), the chemical evolution of galaxies is governed by momentum-driven gas outflows, which are more efficient in expelling metal-enriched gas in dwarf galaxies than in giant galaxies. Spitoni et al. (2010) also support the ejection scenario caused by galactic winds. Köppen et al. (2007) claim that the MZ relation is linked to the initial mass function, which in turn presents a higher upper mass cutoff in more massive galaxies. Calura et al. (2009) advocate that the MZ relation is intrinsically related to the mass and morphological type of the galaxies. They support an increasing efficiency of star formation with mass without any need to invoke galactic outflows. The inflows scenario, whether smooth or in the form of gas-rich satellites, has been explored by Davé et al. (2010).

In this context, several cluster-related environmental processes can affect the star formation history or the gas exchange between the galaxy and its environment and, as a consequence, could alter the chemical evolution of cluster galaxies. Some processes affect mainly the gaseous content of a galaxy, such as the ram-pressure stripping Gunn \& Gott 1972; Kennev et al. 2004; van Gorkom 2004), re-accretion of the stripped gas (Vollmer et al. 2001), turbulence and viscosity (e.g. Quilis et al. 2000) and starvation/strangulation (Larson et al. 1980). Other processes can affect both the gaseous and the stellar properties of a galaxy, ranging from low-velocity tidal interactions and mergers (e.g. Mamon 1996; Barnes \& Hernquist 1996; Conselice 2006) to high-velocity interactions between galaxies and/or clusters ("harassment", Moore et al. 1998, 1999; Struck 1999; Mihos 2004); see Boselli \& Gavazzi (2006) for an extensive review on this subject.

A number of works have been devoted to searching for the possible effects of cluster environment on the chemical properties of spiral galaxies in Virgo cluster (Shields et al. 1991; Henry et al. 1992, 1994, 1996; Skillman et al. 1996; Pilyugin et al. 2002). The spirals at the periphery of the cluster are indistinguishable from the field galaxies, whereas for the HI deficient Virgo spirals near the core it has been claimed that their abundances are higher than for the spirals located at the periphery of the cluster or in the field with comparable luminosity or Hubble type. On the other hand, several works devoted to the study of the star-formation and the metal content of dwarfs in nearby clusters such as Virgo, Coma, and Hydra have revealed that some of them show a different behavior with respect to field galaxies, and this fact has been attributed to the effect of the cluster environment (Vilchez 1995; Duc et al. 2001; Iglesias-Páramo et al. 2003; Vílchez \& Iglesias-Páramo 2003; Lee et al. 2003; Vaduvescu et al. 2007; Smith et al. 2009; Mahajan et al. 2010).

In the era of the large surveys, observational clues which constrain galaxy evolution scenarios in dense environments have lately started to be investigated on the basis of large samples of galaxies. Mouhcine et al. (2007) suggested that the evolution of SF galaxies is driven primarily by their intrinsic properties and is largely independent of their environment over a large range of local galaxy density. On the contrary, Cooper et al. (2008) find a strong relationship between metallicity and environment, such that more metalrich galaxies favor regions of higher overdensity. Ellison et al. (2009) also support the environmental influence, and specially of the local density, on the MZ relation. It is clear that a full understanding of the role played by the environ- 
ment on galaxy evolution still remains a major issue. All these works based on SDSS spectroscopic data present a deficit of important observational information on dwarf cluster galaxies. In addition, SDSS provides aperture spectroscopy, thus missing spatially resolved information that can be much needed, e.g. to analyze the spatial properties of galaxies in clusters.

In this paper we focus our attention on the nearby Hercules (A2151) cluster of galaxies and we investigate the impact of the cluster environment on the chemical history of galaxies. The Hercules cluster shows an amazing variety of different environments found in different parts of the cluster, which makes it one of the more interesting nearby (at a distance of $158.3 \mathrm{Mpc}$ ) dense environments, never studied before from the chemical evolution point of view. It is the richest and densest among the three members of the Hercules supercluster (A2151, A2147, and A2152, Barmby \& Huchra 1998) and appears to be a collection of groups, gravitationally bound but far from dynamical relaxation (Bird et al. 1995; Maccagni et al. 1995). Sánchez-Janssen et al. (2005) report the presence of at least three distinct regions with a varying dwarf to giant galaxy ratio within the A2151 cluster. Giovanelli et al. (1981) studied the supercluster neutral hydrogen content and found that A2147 is clearly deficient in contrast with A2151 which shows normal to mildly gas deficient galaxy population, concluding that A2151 may still be in the process of formation. Dickey (1997) reported the presence of strong environmental effects on the mass of HI in the A2151 cluster. High-Resolution ROSAT data (Huang \& Sarazin 1996; Bird et al. 1995) reveal an irregular X-ray emission pattern which divides this cluster into two main components, which correspond to two groups of galaxies in the region. The maximum of the cluster galaxy distribution is not coincident with the primary $\mathrm{X}$ ray maximum, but with a secondary one located 10-15 arcmin to the East of the primary. This implies that most A2151 galaxies are not expected to be embedded in a hot X-ray intra-cluster medium (ICM). Thus, Hercules cluster provides an ideal case in which the different effects of the cluster environment on the evolution of the galaxies can be studied "caught in the act".

In this work we have benefited from our deep search for $\mathrm{H} \alpha$ emitting galaxies in the Hercules cluster (Cedrés et al. 2009, C09 from now on) to define a new sample of SF galaxies. Tracing $\mathrm{H} \alpha$ emission in order to study the population of galaxies with recent star formation has two important advantages: 1) it is not aperture biased as many of the spectroscopic studies using fibers and 2) it reveals star forming dwarfs which would not have been classified as SF galaxies with an optical flux criterion. We investigate gas-phase metallicities and stellar properties of galaxies which span a large range of magnitudes $\left(-21 \leq \mathrm{M}_{\mathrm{B}} \leq-16.5\right.$ mag) and morphologies (from grand design spirals to blue compacts and tidal dwarfs).

The present paper is organized as follows: in $\S 2$ we present the characteristics of our spectroscopic sample, the observations and the data reduction. In $\S 3$ we present the spectral synthesis techniques performed to compute characteristic properties of the underlying stellar population. In $\S 4$ we derive the chemical and physical properties of our sample of galaxies and in $\S 5$ we discuss the environmental effects on the Hercules cluster galaxies. $\S 6$ summarizes the overall picture of the Hercules cluster as revealed by the chemical evolution point of view.

\section{OBSERVATIONS}

\subsection{The galaxy sample}

Spatially resolved spectroscopy has been obtained for 27 Hercules SF galaxies of the C09 Hercules sample1. We complement our sample with two more galaxies of the C09 sample which have SDSS spectroscopy. In addition, two more galaxies are considered from Iglesias-Páramo et al. (2003) (IP03 from now on) A2151 sample of dwarf galaxies (see section 4.2 for more details). Thus our spectroscopic sample comprises a total of 31 galaxies and our spectroscopic follow-up of the C09 sample is completed up to an absolute magnitude $\mathrm{M}_{\mathrm{B}}=-17$ mag. To illustrate this, in Fig. 1] we show the histogram of the C09 sample of SF galaxies as a function of their $\mathrm{M}_{\mathrm{B}}$ absolute magnitude and the fraction of the galaxies with no spectro-

\footnotetext{
${ }^{1}$ From our spectroscopic data it is shown that the radial velocity of one (SDSS J160547.17+173501.6) of the C09 galaxies is out of the velocity range of the Hercules cluster, rendering its membership to the cluster unlikely. The various SDSS photo-z estimates were consistent with the criteria adopted by C09 for cluster membership. More details are given in Appendix.
} 
scopic data.

Our sample is composed of 15 luminous SF galaxies $\left(\mathrm{M}_{\mathrm{B}} \leq-19\right)$ and 16 dwarf and Magellanic type irregular galaxies $\left(\mathrm{M}_{\mathrm{B}}>-19\right)$. Among the spiral/irregular galaxies, 9 show discernible spatial structure and therefore their different parts have been analyzed independently (see also $\$ 2.3$ and Fig. 18). For three luminous galaxies and three dwarfs/irregulars we have gathered together evidences that they are affected/perturbed from galaxy-galaxy interactions, either being members of mergers, tidal dwarf candidates, or their HI morphology showing evidences of interaction (we give details of these objects in the Appendix).

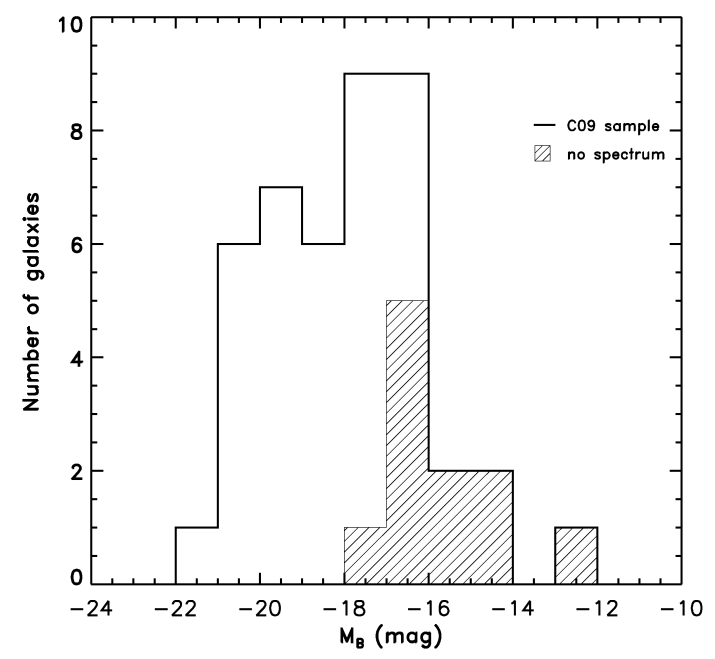

Fig. 1. - The histogram of the C09 sample of SF galaxies as a function of their $\mathrm{M}_{\mathrm{B}}$ absolute magnitude where we show the fraction of galaxies with no spectroscopic data (filled region). Our spectroscopic follow-up of the C09 Hercules SF galaxies is complete up to absolute magnitude $\mathrm{M}_{\mathrm{B}}=-17$ mag.

The Hercules cluster shows abundant substructures seen in X-ray emission and broadband imaging (Huang \& Sarazin 1996). The galaxy surface density as well as the velocity space reveals the presence of subclusters in the galaxy distribution (Maccagni et al. 1995; Bird et al. 1995). The extended X-ray emission coming from the hot intra-cluster gas shows an irregular pattern which divides the cluster into two components.
Fig. 2 illustrates the positions of our spectroscopic sample galaxies (pluses and squares) with respect to the density of the ICM (as seen in $\mathrm{X}$-ray surface brightness map from the ROSAT All-Sky Survey; in blue) and the galaxy surface density of the cluster (derived counting Hercules members 2 with SDSS spectroscopy; red color). There is a primary X-ray maximum concentric with the the brighter cluster galaxy NGC6041A (Huang \& Sarazin 1996). Though, most $\mathrm{H} \alpha$ emitters are located around the secondary X-ray maximum, in the regions of higher density of A2151 galaxies (indicated by the NED position of the mass center; blue cross) within the central region $<R_{200}$ of the Hercules cluster 3 Open squares mark the dwarf/irregular galaxies with radial velocity different from the radial velocity of NGC6041A $\Delta V$ larger than $\sigma \mathrm{V}$, the radial velocity dispersion 4 of Hercules cluster, i.e. $\operatorname{abs}(\Delta \mathrm{V})>$ $\sigma_{\mathrm{V}}$, a possible indication of an infalling population as we will discuss in 5.1 and 5.3 .1 .

\subsection{Optical spectroscopy}

Long-slit spectroscopy was carried out at the $4.2 \mathrm{~m} \mathrm{WHT}$ and the $2.5 \mathrm{~m}$ INT at the Observatorio del Roque de los Muchachos (ORM), in Spain. Table 1 shows the journal of the spectroscopic observations. We present for each galaxy the object name as quoted by C09, RA, DEC, observation date, telescope, effective wavelength range, slit position angle and total exposure time. Most of the galaxies were observed as closer to the zenith as possible, always with air-mass less than 1.3, in order to minimize any differential atmospheric refraction effects; except in four cases, as indicated in Table1, for which the paralactic angle was used.

Observations at the WHT were performed during two observing runs on June and July 2009, using the double arm spectrograph ISIS with slightly different instrumental set-ups. On June 2009 the dichroic splitting the beam was set at $5300 \AA$ and the gratings used were R300B and R158R giving nominal dispersions of $0.86 \AA \mathrm{pix}^{-1}$ and 1.82 $\AA$ pix $^{-1}$ respectively and a spectral coverage of

\footnotetext{
${ }^{2}$ within the velocity range of the subclusters of A2151 defined in Bird et al. (1995)

${ }^{3} R_{200}=1.4 \mathrm{Mpc}$ for the Hercules cluster, following Finn et al. (2005) equation 8 .

${ }^{4}$ The radial velocity dispersion of the Hercules cluster $\sigma_{\mathrm{V}}=$ $752 \mathrm{~km} \mathrm{~s}^{-1}$ (Bird et al. 1995).
} 


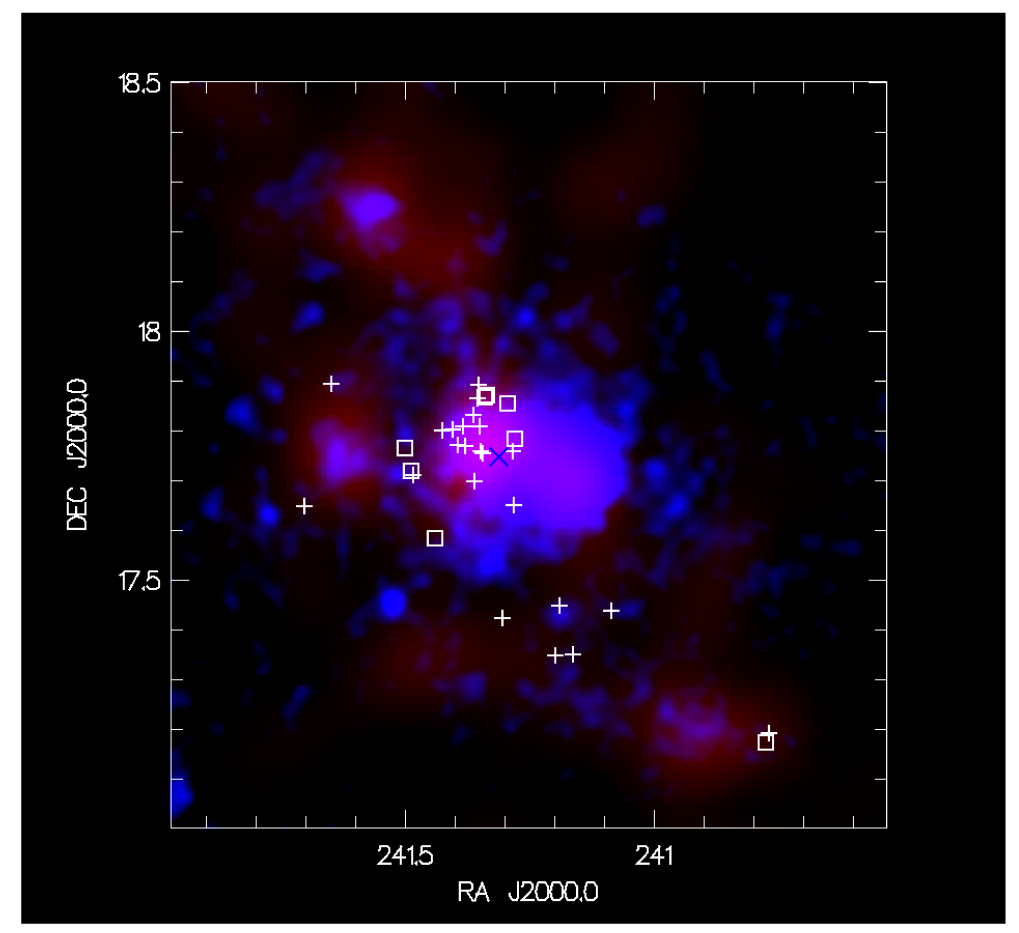

Fig. 2.- Superposition of the map of galaxy density of the Hercules cluster (red) and the ROSAT All-Sky Survey X-ray map (blue). The density map includes all Hercules galaxies with SDSS spectroscopy. The SF galaxies of the present sample are indicated by pluses and squares. Open squares indicate dwarf galaxies with $\operatorname{abs}(\Delta \mathrm{V})>\sigma_{V}$. The surface density of galaxies reveals the presence of subclusters. The extended X-ray emission coming from the hot intra-cluster gas shows an irregular pattern which divides the cluster into two components. Most $\mathrm{H} \alpha$ emitters are located around the secondary X-ray maximum, in the regions of higher density of the A2151 galaxies. The blue cross indicates the position of the cluster center given in NED. See the text for details. 
$\sim 3600-9230 \AA$ A. On July 2009 the dichroic was set at $5700 \AA$ and the gratings used were R300B and R316R giving respective dispersions 0.83 and 0.89 $\AA$ pix $^{-1}$ and a spectral coverage $\sim 3160-8263 \AA$. During both runs the CCD set-up was the same: on the blue arm the EEV12 detector, providing a spatial scale 0.19 arcsec $\mathrm{pix}^{-1}$, and on the red arm the RED+ detector sampling 0.22 arcsec $\mathrm{pix}^{-1}$. Observations at the INT were also performed during two observational runs, on June 2008 and May 2009, using the Intermediate Dispersion Spectrograph (IDS) with the EEV10 detector and R300V grating, giving a nominal dispersion of $1.86 \AA$ $\mathrm{pix}^{-1}$ and a spatial scale of $0.4 \operatorname{arcsec} \mathrm{pix}^{-1}$. The total wavelength coverage free of vignetting with this set-up was $\sim 3400-7700 \AA$. In both ISIS and IDS spectra the slit width was set to 1.5 arcsec. The orientation of the slit, as listed in Table 1 was selected to cover the maximum surface of the $\mathrm{H} \alpha$ emission detected by Cedrés et al. (2009), with few exceptions that were observed on paralactic angle.

\subsection{Data reduction}

The spectra were reduced in the standard manner using IRAF routines. First, the twodimensional spectra were bias subtracted and flat-field corrected. For this, dome flat exposures were used to remove pixel-to-pixel variations in response. Wavelength calibration was achieved using spectra of $\mathrm{CuNe}$ and $\mathrm{CuAr}$ comparison lamps, reaching an accuracy of $\sim 0.3 \AA$ for IDS and less than $0.1 \AA$ for ISIS spectra. Several exposures were taken for each object in order to remove cosmic ray hits. The spectra were corrected for atmospheric extinction using an extinction curve for ORM (King 1985) and all observing nights were photometric, according to Carlsberg Meridian Telescope records. Enough slit length free of any object permitted an adequate sky-subtraction. For the flux calibration, during the observational runs each night at least 3 spectrophotometric standard stars were observed, varying in color, except during the 26-27 June 2009 when we observed two and one stars respectively. The expected spectrophotometric error is estimated 10\% (June 2008) 8\% (May 2009) 3\% (June 2009) and 2\% (July 2009). ISIS matching between red and blue arm spectra was successful as can be seen in the spectra shown in Fig. 17] in the Appendix.
From the 2D frames an integrated 1D spectrum was produced for those galaxies showing no significant spatial structure. Conversely for galaxies showing rich spatial structure, their 2D spectrum is divided into different $1 \mathrm{D}$ spectra corresponding to their different sub-regions. For these galaxies, in Fig. 18 in the Appendix we show g-i color maps produced using SDSS images and $\mathrm{H} \alpha$ equivalentwidth (EW) maps using our data, with the precise position of the slit overploted on both maps. We also give the spatial profiles of the $\mathrm{H} \alpha$ line and the nearby continuum emission along the slit position extracted from the $2 \mathrm{D}$-spectra. On these profiles the different galaxy parts considered in our analysis are highlighted. All the 1D spectra extracted in this study are presented in Fig. 17 in the Appendix.

\section{SPECTRAL SYNTHESIS MODEL FITTING}

We use the population synthesis code STARLIGHT to fit spectral synthesis models to all our spectra and we compute some characteristic properties of the underlying stellar component as the luminosity-weighted and mass-weighted stellar age and metallicity (see, e.g. Asari et al. 2007). We also used the model fits to correct our spectra for underlying stellar absorption. Our galaxy sample spans a considerable range in its emissionline properties, from systems with Balmer emission lines with large equivalent widths to systems where the $\mathrm{H} \beta$ is just detectable above a high level of stellar continuum. Especially in the latter cases, an accurate correction for underlying stellar absorption is crucial. The usual practice of adopting an $\mathrm{EW}_{\mathrm{abs}}$ of $\sim 2 \AA$ for stellar Balmer lines, though generally justifiable, bears the risk of introducing significant systematic errors in the analysis of systems with faint emission lines. This is because the exact value for $\mathrm{EW}_{\mathrm{abs}}$ depends on the stellar population mixture i.e. the star formation history (SFH) in a galaxy. For example, Gonzalez Delgado et al. (1999) infer for a single stellar population (SSP) a monotonous increase of

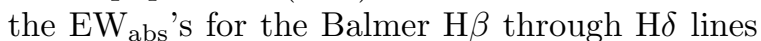
from $\lesssim 3 \AA$ at an age $\leq 3$ Myr to $>10 \AA$ at ages $\sim 0.5 \mathrm{Gyr}$, followed by a decrease for older ages. Star-forming galaxies, consisting of different SSPs of young to intermediate age, in addition to a (significantly) older underlying host galaxy, likely fol- 
TABLE 1

LOG OF OBSERVATIONS

\begin{tabular}{|c|c|c|c|c|c|c|c|}
\hline $\begin{array}{c}\text { Galaxy } \\
\text { (1) }\end{array}$ & $\begin{array}{c}\text { RA } \\
\text { J2000.0 } \\
(2)\end{array}$ & $\begin{array}{c}\text { DEC } \\
\text { J2000.0 } \\
(3)\end{array}$ & $\begin{array}{l}\text { Date } \\
(4)\end{array}$ & $\begin{array}{l}\text { Tel. } \\
(5)\end{array}$ & $\begin{array}{c}\text { Range } \\
\text { (6) }\end{array}$ & PA & $\begin{array}{r}\text { Exp. } \\
(8)\end{array}$ \\
\hline PGC057185 & 160648.2 & 173851.6 & 2008 Jun 06 & INT & $3500-7730$ & 13 & 3600 \\
\hline IC1173 & 160512.6 & 172522.3 & 2008 Jun 06 & INT & $3500-7730$ & 45 & 3600 \\
\hline KUG1603+179A & 160530.6 & $1746 \quad 07.2$ & 2008 Jun 06 & INT & $3500-7730$ & 30 & 4500 \\
\hline NGC6050A, $B^{\ddagger}$ & $\begin{array}{lll}16 & 0523.4\end{array}$ & 174525.8 & 2008 Jun 06 & INT & $3500-7730$ & $67^{\dagger}$ & 3600 \\
\hline LEDA1543586 & 160510.5 & 175116.1 & 2008 Jun 07 & INT & $3500-7730$ & 297 & 5300 \\
\hline NGC6045 & 160507.9 & 174527.6 & 2008 Jun 07 & INT & $3500-7730$ & 75 & 4500 \\
\hline KUG1602+174A & 160439.0 & 172059.9 & 2008 Jun 07 & INT & $3500-7730$ & $59^{\dagger}$ & 5400 \\
\hline LEDA084719 & $\begin{array}{lll}16 & 05 & 07.1\end{array}$ & 173857.8 & 2008 Jun 07 & INT & $3500-7730$ & $67^{\dagger}$ & 4200 \\
\hline PGC057077 & 160534.2 & 174611.8 & 2008 Jun 08 & INT & $3500-7730$ & 297 & 4500 \\
\hline UGC10190 & 160526.3 & 174148.6 & 2008 Jun 08 & INT & $3500-7730$ & 150 & 4500 \\
\hline LEDA140568 & $\begin{array}{lll}16 & 06 & 00.1\end{array}$ & 174552.0 & 2008 Jun 08 & INT & $3500-7730$ & 10 & 5400 \\
\hline$[D 97] \mathrm{ce}-200$ & 160506.8 & 174702.0 & 2008 Jun 08 & INT & $3500-7730$ & $66^{\dagger}$ & 5400 \\
\hline PGC057064 & 160527.2 & 174951.6 & 2008 Jun 09 & INT & $3500-7730$ & 115 & 5400 \\
\hline LEDA084703 & 160305.7 & 171020.4 & 2008 Jun 09 & INT & $3500-7730$ & 82 & 5400 \\
\hline KUG1602+175 & 160445.4 & 172654.3 & 2009 May 26 & INT & $3400-7630$ & 315 & 3600 \\
\hline LEDA084710 & 160420.4 & 172611.2 & 2009 May 26 & INT & $3400-7630$ & 105 & 3600 \\
\hline CGCG108-149 & 160635.3 & 175333.2 & 2009 May 26 & INT & $3400-7630$ & 172 & 3600 \\
\hline KUG1602+174B & 160447.6 & 172052.0 & 2009 May 26 & INT & $3400-7630$ & 67 & 2400 \\
\hline LEDA084724 & 160545.5 & 173456.7 & 2009 Jun 26 & WHT & $3600-9230$ & 0 & 3000 \\
\hline SDSS J160556.98+174304.1 & 160557.0 & 174304.1 & 2009 Jun 26 & WHT & $3600-9230$ & 90 & 3600 \\
\hline [DKP87]160310.21+175956.7 & 160525.0 & 175150.6 & 2009 Jun 26 & WHT & $3600-9230$ & 114 & 3600 \\
\hline SDSS J160524.27+175329.3 & 160524.3 & 175329.4 & 2009 Jun 27 & WHT & $3600-9230$ & 102 & 3600 \\
\hline SDSS J160547.17+173501.6 & 160547.2 & 173501.7 & 2009 Jun 27 & WHT & $3600-9230$ & 170 & 3600 \\
\hline SDSS J160523.66+174832.3 & 160523.7 & 174832.3 & 2009 Jun 27 & WHT & $3600-9230$ & 140 & 3600 \\
\hline IC1182 & 160536.8 & 174807.5 & 2009 Jul 19 & WHT & $3160-8263$ & 127 & 3000 \\
\hline IC1182:[S72]d & 160541.9 & 174758.4 & 2009 Jul 19 & WHT & $3160-8263$ & 96 & 3600 \\
\hline SDSS J150531.84+174826.1 & 160531.8 & 174826.2 & 2009 Jul 19 & WHT & $3160-8263$ & 66 & 3600 \\
\hline
\end{tabular}

Note.-Column 1: Galaxy name following Cedrés et al. (2009); Column 2: right ascension in hours, minutes, and seconds; Column 3: declination, in degrees, arcminutes, and arcseconds; Column 4: observation date; Column 5: telescope used; Column 6: wavelength range coverage in $\AA$; Column 7: position angle in degrees; Column 8: exposure time in seconds.

${ }^{\dagger}$ Observed at paralactic angle through air-mass $\geq 1.3$.

$\ddagger_{\text {This }}$ pair is also known as Arp 272. 
low a considerably more complex time evolution in their luminosity-weighted $\mathrm{EW}_{\mathrm{abs}}$. This issue has been explored in much detail by e.g. Guseva et al. (2001) who have considered a variety of SFHs and mass proportions between the young and older stellar components in SF dwarf galaxies. Obviously, for systems with low emission-line EWs, improper correction for underlying stellar absorption may propagate into significant errors in e.g., the intrinsic extinction or spectroscopic classification using BPT (Baldwin et al. 1981) diagrams. For example, for an intermediate luminosity-weighted age of $0.2-0.6 \mathrm{Gyr}$, where Balmer $\mathrm{EW}_{\mathrm{abs}}$ are largest, the underestimation of Balmer line fluxes due to incomplete correction for underlying stellar absorption may shift a star forming galaxy on the $\log ([\mathrm{OIII}] \lambda 5007 / \mathrm{H} \beta) \quad$ vs. $\quad \log ([\mathrm{NII}] \lambda 6583 / \mathrm{H} \alpha)$ $\mathrm{BPT}$ diagram into the zone of active galactic nuclei (AGNs).

The determination of the $\mathrm{EW}_{\mathrm{abs}}$ may be carried out by simultaneous fitting of two Gaussians to each emission and absorption Balmer line profile. Another adequate, and arguably more efficient, technique for correcting for underlying stellar absorption is the fitting and subsequent subtraction of the observed spectrum of a synthetic stellar spectrum in order to isolate the net nebular line emission fluxes. To this end, we used the population synthesis code STARLIGHT (Cid Fernandes et al. 2004, 2005a,b; Garcia-Rissmann et al. 2005). This code synthesizes the observed stellar continuum as due to the superposition of SSPs of different age and metallicity. The SSP library used is based on stellar models by Bruzual \& Charlot (2003) for a Salpeter initial mass function (IMF) for three metallicities $\left(Z_{\odot} / 5, Z_{\odot} / 2.5\right.$ and $\left.Z_{\odot}\right) \sqrt{5}$ and 59 ages (between 0.25 Myr and $13 \mathrm{Gyr}$ ). Output of the model is the list of those SSPs with a mass contribution $M_{i}>0(\%)$ and their luminosity contribution $L_{i}(\%)$ to the wavelength interval to which the input spectra have been normalized (5100-5300 $\AA$ here).

Prior to modeling the observed spectra were deredshifted and resampled to $1 \AA$ /pixel. Additionally, emission lines and residuals from the night

\footnotetext{
${ }^{5}$ The solar metallicity is assumed $Z_{\odot}=0.019$, which is translated to an oxygen abundance $12+\log (O / H)_{\odot}=8.69$ (Asplund et al. 2009).
}

sky subtraction were identified and interactively flagged using a code developed by us. Throughout, we preferred not to correct the input spectra for intrinsic extinction or to strongly constrain its allowed range in the STARLIGHT models for a twofold reason: first, the extinction coefficient $\mathrm{C}(\mathrm{H} \beta)$ computed prior to correction of Balmer emission line fluxes for underlying stellar absorption is, especially for galaxies with faint nebular emission, uncertain. An a priori de-redenning of the input spectra using this approximate $\mathrm{C}(\mathrm{H} \beta)$ value would therefore yield no advantage towards reliability and computational expense. For the same reason, and in order to minimize biases in the STARLIGHT solutions, we have allowed the intrinsic extinction to vary between +4.5 and -0.7 $\mathrm{V}$ mag. The allowance for a negative $A_{V}$ is justified by possible uncertainties in flux calibration, the (partial) coverage of the physical spatial area of the galaxy, as well as the incompleteness of the SSP library. As evident from Table 2 solutions with $A_{V} \lesssim 0$ are the exception in our analysis. Secondly, fixing the intrinsic extinction in the STARLIGHT models to the observed $\mathrm{C}(\mathrm{H} \beta)$ would imply that ionized gas and stars are subject to equal extinction. No compelling evidence exists for this as yet. On the other hand, for the sake of simplicity and to keep computational time low, we have throughout assumed a single foreground extinction model, although provision is given in STARLIGHT to solve for a different extinction in the young ( $\leq 10 \mathrm{Myr})$ and older stellar population.

A typical example of the spectral synthesis for a galaxy with relatively faint nebular emission in our sample is shown in Fig. 3. The left panel shows the observed, normalized spectrum (orange color) with the best-fitting synthetic spectrum overlaid (blue color). Flagged intervals in each spectrum are marked with shaded vertical bars. The smaller panels to the upper-right and lower-right show the age distribution of the SSPs evaluated by STARLIGHT as a function of, respectively, their luminosity and mass fraction in \%. Thin vertical lines in the upper right panel indicate the ages of the library SSPs. The shaded area in the lower diagram shows the smoothed $M_{i}$ distribution and is meant as schematic illustration of the SFH. It may be seen from the left panel that synthetic spectra fit well broad stellar absorption features, thus permitting an adequate determination and correction 
for underlying absorption. The corrected emission line fluxes, as measured from the observed spectra after subtraction of the synthetic stellar spectra are listed in Table 3 (see $\$ 4$ for a detailed discussion).

Although an elaborated study of the SFH of the sample galaxies is beyond the scope of this paper, we took advantage of the STARLIGHT models to compute some characteristic properties of our sample galaxies. These include the luminosityweighted and mass-weighted metallicity $Z_{\star, L}$ and $Z_{\star, M}$ and stellar age $\tau_{\star, L}$ and $\tau_{\star, M}$ along with their standard deviations (see, e.g., Asari et al. 2007). These quantities and the intrinsic extinction $A_{V}$ of the stellar component, are listed in Table 2 and will be further discussed in $\$ 5$.

\section{RESULTS}

\subsection{Line fluxes}

Line fluxes were measured in the spectra after subtracting the best-fitting spectral energy distribution (SED) of the underlying stellar population (see 93 ) using the SPLOT task of IRAF. Line fluxes were measured integrating the flux under the line profile over a linearly fitted continuum. In order to account for the main source of error in our data, driven by the continuum placement, five independent measurements of the emission line flux were carried out for each line and we assume the mean and the standard deviation as the flux measurement and the corresponding statistical error.

The reddening coefficients $\mathrm{c}(\mathrm{H} \beta)$ were calculated adopting the galactic extinction law of Miller \& Mathews (1972) with $R_{V}=3.2$, as presented in Hägele et al. (2008), using the expression:

$$
\log \left[\frac{\mathrm{I}(\lambda)}{\mathrm{I}(\mathrm{H} \beta)}\right]=\log \left[\frac{\mathrm{F}(\lambda)}{\mathrm{F}(\mathrm{H} \beta)}\right]+\mathrm{c}(\mathrm{H} \beta) \mathrm{f}(\lambda)
$$

where $\mathrm{I}(\lambda) / \mathrm{I}(\mathrm{H} \beta)$ is the theoretical and $\mathrm{F}(\lambda) / \mathrm{F}(\mathrm{H} \beta)$ the observed Balmer decrements. The theoretical Balmer decrement depends on electron temperature and density and we used the data for Case $\mathrm{B}$ and assuming low-density limit and 10,000K (Storey \& Hummer 1995). In those cases where we measured at least three Balmer lines with $S / N>5$ we have performed a least-squares fit to derive $\mathrm{c}(\mathrm{H} \beta)$ and the corresponding error was taken to be the uncertainty of the fit. When only
$\mathrm{H} \alpha$ and $\mathrm{H} \beta$ were available, $\mathrm{c}(\mathrm{H} \beta)$ was derived applying expression (1) and its error was calculated as the rms of a gaussian error distribution produced by a random sampling simulation, taking into account the errors in the line fluxes of $\mathrm{H} \alpha$ and $\mathrm{H} \beta$.

Reddening-corrected line fluxes and reddening coefficients $\mathrm{c}(\mathrm{H} \beta)$ are presented in Table 3 The errors quoted account for the uncertainties in the line flux measurement and in the extinction coefficient. Table 3 also presents the $\mathrm{H} \beta$ flux not corrected for extinction and equivalent width, as well as the galaxy recessional velocity, as derived by our spectroscopic data. The $\mathrm{H} \beta$ equivalent width quoted was determined from the net emission spectrum, i.e. the observed spectrum after subtraction of the best-fitting stellar SED, and the level of the adjacent continuum in the original spectrum.

Fig. 4 shows the extinction of the gas-phase component $A_{V, \text { gas }}=2.18 \times \mathrm{c}(\mathrm{H} \beta)$ as a function of the intrinsic extinction of the stellar component derived by STARLIGHT in the previous section. A linear fit, considering (the reliable range, see (3) $A_{V, \text { star }}>-0.1$ and weighted by the errors of the $\mathrm{c}(\mathrm{H} \beta)$ estimation, yield the relation $A_{V, \text { gas }}=0.81+0.89 A_{V, \text { star }}$. We find that stars suffer less extinction than gas, that can be explained if the dust has a larger covering factor for the ionized gas than for the stars (Calzetti et al. 1994). In the same line are recent results presented by Monreal-Ibero et al. (2010) and Alonso-Herrero et al. (2010), who explore the relation between the extinction suffered by the gas and by the stellar populations in the central regions of SF galaxies using IFU data.

\subsection{Comparison with previous data}

SDSS spectroscopic data exist for 22 out of the $43 \mathrm{SF}$ galaxies of the C09 sample. In most of the cases the SDSS fiber covers the central 3 arcsec of the galaxy; for 3 galaxies, NGC6050, NGC6045 and PGC057064, SDSS provides 2 different apertures on each of them. We have applied the same procedure to these spectra as to our spatially resolved spectra, removing the continuum emission by fitting STARLIGHT and measuring line fluxes on the subtracted emission-line spectrum as described in 83 and 4.1. We then have compared these line fluxes with our line fluxes for 19 galax- 
TABLE 2

STARLIGHT OUTPUT

\begin{tabular}{|c|c|c|c|c|c|}
\hline Galaxy & $Z_{\star, L}$ & $Z_{\star, M}$ & $\begin{array}{l}\tau_{\star, L} \\
\text { Gyr }\end{array}$ & $\begin{array}{c}\tau_{\star}, M \\
\mathrm{Gyr}\end{array}$ & $\begin{array}{c}A_{V} \\
\text { mag }\end{array}$ \\
\hline PGC05185a & $0.014 \pm 0.008$ & $0.012 \pm 0.008$ & $0.94 \pm 0.30$ & $1.08 \pm 0.28$ & 0.98 \\
\hline PGC05185b & $0.009 \pm 0.005$ & $0.007 \pm 0.004$ & $2.02 \pm 1.02$ & $7.28 \pm 3.07$ & 1.68 \\
\hline PGC05185c & $0.014 \pm 0.007$ & $0.009 \pm 0.004$ & $2.64 \pm 1.24$ & $8.54 \pm 2.07$ & 1.32 \\
\hline IC1173a & $0.016 \pm 0.006$ & $0.019 \pm 0.009$ & $7.73 \pm 1.92$ & $11.10 \pm 0.80$ & -0.21 \\
\hline IC $1173 \mathrm{e}$ & $0.018 \pm 0.009$ & $0.016 \pm 0.007$ & $2.09 \pm 0.72$ & $5.66 \pm 1.86$ & 0.63 \\
\hline KUG1603+179Aa & $0.014 \pm 0.007$ & $0.012 \pm 0.005$ & $3.69 \pm 1.35$ & $6.60 \pm 1.81$ & -0.56 \\
\hline KUG1603+179Ab & $0.015 \pm 0.006$ & $0.018 \pm 0.011$ & $7.27 \pm 2.25$ & $11.62 \pm 0.76$ & 0.91 \\
\hline KUG1603+179Ac & $0.012 \pm 0.004$ & $0.008 \pm 0.005$ & $4.80 \pm 1.98$ & $11.07 \pm 0.77$ & 1.09 \\
\hline NGC6050Aa & $0.011 \pm 0.004$ & $0.014 \pm 0.005$ & $8.10 \pm 1.93$ & $11.36 \pm 0.58$ & 0.47 \\
\hline NGC6050Ab & $0.013 \pm 0.003$ & $0.012 \pm 0.005$ & $5.67 \pm 1.87$ & $11.58 \pm 0.54$ & 0.41 \\
\hline NGC6050B & $0.007 \pm 0.002$ & $0.004 \pm 0.002$ & $5.82 \pm 1.35$ & $8.54 \pm 0.97$ & -0.12 \\
\hline LEDA1543586 & $0.006 \pm 0.002$ & $0.008 \pm 0.003$ & $1.98 \pm 0.91$ & $6.66 \pm 1.71$ & 0.42 \\
\hline NGC6045a & $0.015 \pm 0.005$ & $0.018 \pm 0.010$ & $4.87 \pm 1.94$ & $10.82 \pm 1.20$ & 0.57 \\
\hline NGC6045b & $0.013 \pm 0.004$ & $0.017 \pm 0.008$ & $5.42 \pm 1.74$ & $10.10 \pm 1.31$ & 0.27 \\
\hline NGC6045c & $0.016 \pm 0.008$ & $0.017 \pm 0.013$ & $8.30 \pm 2.45$ & $12.68 \pm 0.28$ & 2.13 \\
\hline NGC6045d & $0.013 \pm 0.005$ & $0.015 \pm 0.008$ & $4.25 \pm 1.78$ & $11.14 \pm 1.38$ & 1.33 \\
\hline NGC6045e & $0.011 \pm 0.007$ & $0.010 \pm 0.008$ & $1.06 \pm 0.30$ & $2.71 \pm 1.60$ & 0.93 \\
\hline KUG1602+174A & $0.007 \pm 0.002$ & $0.008 \pm 0.003$ & $4.66 \pm 1.34$ & $8.34 \pm 0.87$ & 0.19 \\
\hline LEDA084719 & $0.008 \pm 0.004$ & $0.012 \pm 0.004$ & $4.45 \pm 1.41$ & $8.34 \pm 1.53$ & 0.15 \\
\hline PGC057077a & $0.013 \pm 0.006$ & $0.018 \pm 0.017$ & $4.24 \pm 2.86$ & $12.03 \pm 1.19$ & 3.23 \\
\hline PGC057077b & $0.014 \pm 0.009$ & $0.013 \pm 0.008$ & $1.75 \pm 0.41$ & $2.10 \pm 0.60$ & -0.70 \\
\hline UGC10190a & $0.007 \pm 0.003$ & $0.005 \pm 0.002$ & $3.43 \pm 1.46$ & $8.14 \pm 1.91$ & 0.22 \\
\hline UGC10190b & $0.007 \pm 0.002$ & $0.006 \pm 0.002$ & $5.85 \pm 1.22$ & $10.04 \pm 0.71$ & 0.79 \\
\hline UGC10190c & $0.014 \pm 0.005$ & $0.009 \pm 0.005$ & $5.45 \pm 2.16$ & $11.63 \pm 0.64$ & 0.82 \\
\hline LEDA 140568 & $0.008 \pm 0.004$ & $0.006 \pm 0.003$ & $0.86 \pm 0.45$ & $4.84 \pm 2.17$ & 0.56 \\
\hline D97ce200 & $0.007 \pm 0.003$ & $0.008 \pm 0.005$ & $0.73 \pm 0.25$ & $1.09 \pm 0.11$ & 0.33 \\
\hline PGC057064a & $0.014 \pm 0.005$ & $0.017 \pm 0.008$ & $5.06 \pm 1.36$ & $8.13 \pm 0.85$ & 0.74 \\
\hline PGC057064b & $0.011 \pm 0.004$ & $0.017 \pm 0.007$ & $6.56 \pm 1.75$ & $10.52 \pm 0.80$ & 0.36 \\
\hline LEDA084703int & $0.009 \pm 0.002$ & $0.009 \pm 0.002$ & $1.22 \pm 0.26$ & $2.42 \pm 0.50$ & 0.31 \\
\hline LEDA084703a & $0.007 \pm 0.005$ & $0.008 \pm 0.007$ & $0.71 \pm 0.26$ & $1.00 \pm 0.02$ & -0.34 \\
\hline LEDA084703b & $0.009 \pm 0.003$ & $0.012 \pm 0.004$ & $1.97 \pm 0.52$ & $5.93 \pm 1.60$ & 0.50 \\
\hline LEDA084703c & $0.009 \pm 0.005$ & $0.008 \pm 0.005$ & $0.94 \pm 0.29$ & $1.75 \pm 0.63$ & 0.43 \\
\hline KUG1602+175 & $0.013 \pm 0.006$ & $0.017 \pm 0.007$ & $3.76 \pm 1.54$ & $9.74 \pm 1.55$ & 0.55 \\
\hline LEDA084710 & $0.015 \pm 0.007$ & $0.017 \pm 0.008$ & $2.86 \pm 1.24$ & $9.05 \pm 2.05$ & 1.02 \\
\hline CGCG108-149 & $0.018 \pm 0.008$ & $0.019 \pm 0.010$ & $7.68 \pm 2.46$ & $11.11 \pm 1.08$ & 0.40 \\
\hline KUG1602+174B & $0.016 \pm 0.007$ & $0.018 \pm 0.008$ & $2.91 \pm 1.11$ & $7.76 \pm 1.31$ & 0.48 \\
\hline LEDA084724 & $0.007 \pm 0.003$ & $0.008 \pm 0.003$ & $3.15 \pm 1.24$ & $7.57 \pm 0.92$ & 0.37 \\
\hline SDSSJ160556.98+174304.1 & $0.008 \pm 0.006$ & $0.008 \pm 0.007$ & $0.82 \pm 0.49$ & $1.26 \pm 0.21$ & 0.61 \\
\hline [DKP87]160310.21+175956.7 & $0.006 \pm 0.001$ & $0.006 \pm 0.002$ & $3.11 \pm 1.48$ & $7.72 \pm 0.69$ & 0.31 \\
\hline SDSSJ160524.27+175329.3 & $0.004 \pm 0.004$ & $0.004 \pm 0.004$ & $1.06 \pm 0.21$ & $1.06 \pm 0.10$ & -0.01 \\
\hline SDSSJ160523.66+174832.3 & $0.007 \pm 0.004$ & $0.004 \pm 0.002$ & $2.25 \pm 0.99$ & $6.16 \pm 2.52$ & 0.38 \\
\hline IC1182 & $0.012 \pm 0.004$ & $0.014 \pm 0.008$ & $6.37 \pm 1.97$ & $11.56 \pm 0.88$ & 1.47 \\
\hline IC1182:[S72]d & $0.019 \pm 0.011$ & $0.018 \pm 0.011$ & $1.92 \pm 1.49$ & $11.51 \pm 0.76$ & 0.42 \\
\hline SDSSJ160531.84+174826.1 & $0.007 \pm 0.003$ & $0.008 \pm 0.004$ & $6.11 \pm 2.46$ & $11.10 \pm 1.13$ & 1.11 \\
\hline SDSSJ160304.20+171126.7 & $0.007 \pm 0.002$ & $0.006 \pm 0.002$ & $3.88 \pm 0.71$ & $5.78 \pm 0.63$ & 0.48 \\
\hline SDSSJ160520.58+175210.6 & $0.008 \pm 0.003$ & $0.008 \pm 0.003$ & $5.76 \pm 0.65$ & $7.11 \pm 0.46$ & 0.50 \\
\hline
\end{tabular}

NoTE.- The luminosity-weighted and mass-weighted stellar metallicity (e.g. the mass fraction of metals over $\mathrm{H}$, where the solar value is assumed to be $\left.Z_{\odot}=0.019\right) Z_{\star, L}$ and $Z_{\star}, M$ and stellar age $\tau_{\star, L}$ and $\tau_{\star, M}$ along with their the standard deviations and the intrinsic extinction $A_{V}$ of the stellar component as given by fitting STARLIGHT models. 

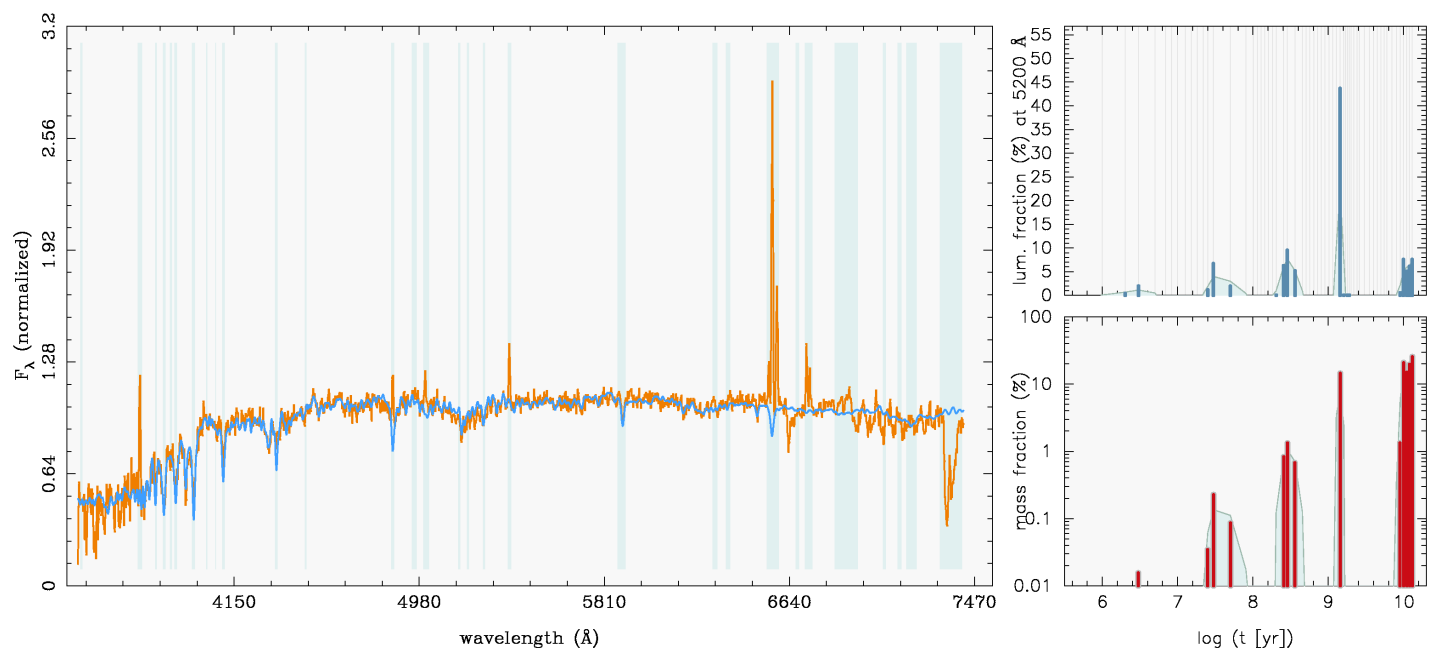

Fig. 3.- A typical example of spectral synthesis with STARLIGHT for the galaxy KUG1602+175. The left panel shows the observed, normalized spectrum (orange color) with the best-fitting synthetic spectrum overlaid (blue color). Flagged intervals in each spectrum are marked with shaded vertical bars. The smaller panels to the upper-right and lower-right show the age distribution of the SSPs evaluated by STARLIGHT as a function of, respectively, their luminosity and mass fraction in \%. Thin vertical lines in the upper right panel indicate the ages of the library SSPs. The shaded area in the lower diagram shows the smoothed $M_{i}$ distribution and is meant as schematic illustration of the SFH.

TABLE 3

REDDENING-CORRECTED LINE FLUXES

\begin{tabular}{|c|c|c|c|c|c|c|c|}
\hline \multirow[t]{2}{*}{ Line } & \multirow[t]{2}{*}{$f(\lambda)$} & \multicolumn{3}{|c|}{ PGC057185 } & \multicolumn{3}{|c|}{ IC1173 } \\
\hline & & $\mathrm{a}$ & $\mathrm{b}$ & c & $\mathrm{a}$ & $\mathrm{b}$ & e \\
\hline $3727[\mathrm{O}$ II $] \ldots \ldots \ldots$ & 0.271 & $305 \pm 20$ & $231 \pm 5$ & $239 \pm 53$ & $206 \pm 37$ & $991 \pm 131$ & $118 \pm 9$ \\
\hline $3869[\mathrm{Ne}$ III $] \ldots \ldots$ & 0.238 & $\ldots$ & $\ldots$ & $\ldots$ & $\ldots$ & $\ldots$ & $\ldots$ \\
\hline $3889 \mathrm{He} \mathrm{I}+\mathrm{H} 8 \ldots \ldots$ & 0.233 & $\cdots$ & $\cdots$ & $\cdots$ & $\cdots$ & $\cdots$ & $\cdots$ \\
\hline $3968[\mathrm{Ne}$ III $]+\mathrm{H} 7 \ldots$ & 0.216 & $\cdots$ & $\cdots$ & $\cdots$ & $\cdots$ & $\cdots$ & $\cdots$ \\
\hline $4069[\mathrm{~S}$ II $] \ldots \ldots \ldots$ & 0.195 & $\cdots$ & $\ldots$ & $\cdots$ & $\cdots$ & $\ldots$ & $\ldots$ \\
\hline $4102 \mathrm{H} \delta \ldots \ldots \ldots$ & 0.188 & $\ldots$ & $26 \pm 5$ & $\cdots$ & $\cdots$ & $\cdots$ & $\ldots$ \\
\hline $4340 \mathrm{H} \gamma \ldots \ldots \ldots$ & 0.142 & $50 \pm 16$ & $47 \pm 8$ & $\ldots$ & $\ldots$ & $\ldots$ & $48 \pm 2$ \\
\hline $4861 \mathrm{H} \beta \ldots \ldots \ldots$ & 0.00 & $100 \pm 8$ & $100 \pm 9$ & $100 \pm 18$ & $100 \pm 13$ & $100 \pm 12$ & $100 \pm 7$ \\
\hline $4959[\mathrm{O}$ III $] \ldots \ldots$ & -0.024 & $\ldots$ & $12 \pm 2$ & $\ldots$ & $\ldots$ & $\ldots$ & $\ldots$ \\
\hline $5007[\mathrm{O}$ III $] \ldots \ldots \ldots$ & -0.035 & $57 \pm 4$ & $36 \pm 2$ & $54 \pm 10$ & $25 \pm 15$ & $236 \pm 18$ & $88 \pm 6$ \\
\hline 5876 He I......... & -0.209 & $\ldots$ & $11 \pm 1$ & $\ldots$ & $\ldots$ & $\ldots$ & $\ldots$ \\
\hline $6300[\mathrm{O}$ I $] \ldots \ldots \ldots$ & -0.276 & $21 \pm 5$ & $16 \pm 1$ & $\cdots$ & $\ldots$ & $71 \pm 15$ & $\cdots$ \\
\hline $6548\left[\begin{array}{ll}\mathrm{N} & \mathrm{II}\end{array}\right] \ldots \ldots \ldots$ & -0.311 & $31 \pm 3$ & $39 \pm 6$ & $30 \pm 7$ & $62 \pm 11$ & $180 \pm 26$ & $\ldots$ \\
\hline $6563 \mathrm{H} \alpha \ldots \ldots \ldots$ & -0.313 & $302 \pm 14$ & $294 \pm 8$ & $282 \pm 54$ & $285 \pm 40$ & $285 \pm 41$ & $287 \pm 15$ \\
\hline $6584\left[\begin{array}{ll}\mathrm{N} & \mathrm{II}\end{array}\right] \ldots \ldots \ldots$ & -0.316 & $85 \pm 5$ & $113 \pm 7$ & $94 \pm 18$ & $140 \pm 21$ & $492 \pm 68$ & $103 \pm 10$ \\
\hline $6717[\mathrm{~S}$ II $] \ldots \ldots \ldots$ & -0.334 & $64 \pm 4$ & $46 \pm 4$ & $50 \pm 11$ & $38 \pm 7$ & $157 \pm 24$ & $84 \pm 3$ \\
\hline $6731[\mathrm{~S}$ II $] \ldots \ldots \ldots$ & -0.336 & $55 \pm 4$ & $41 \pm 4$ & $44 \pm 11$ & $54 \pm 19$ & $152 \pm 24$ & $63 \pm 8$ \\
\hline $\mathrm{F}(\mathrm{H} \beta)$ & & $4.8 \pm 0.4$ & $15.7 \pm 1.5$ & $2.1 \pm 0.4$ & $1.7 \pm 0.2$ & $2.3 \pm 0.3$ & $2.2 \pm 0.2$ \\
\hline $\mathrm{EW}(\mathrm{H} \beta)$ & & $6.5 \pm 0.8$ & $5.5 \pm 0.6$ & $3.0 \pm 0.6$ & $1.9 \pm 0.3$ & $1.6 \pm 0.4$ & $8.1 \pm 1.6$ \\
\hline $\mathrm{c}(\mathrm{H} \beta)$ & & $0.70 \pm 0.06$ & $1.09 \pm 0.02$ & $1.29 \pm 0.26$ & $1.02 \pm 0.19$ & $0.96 \pm 0.19$ & $-0.21 \pm 0.29$ \\
\hline velocity & & $11403 \pm 27$ & $11204 \pm 19$ & $11122 \pm 30$ & $10617 \pm 15$ & $11204 \pm 19$ & $10248 \pm 44$ \\
\hline
\end{tabular}

Note. $-\mathrm{F}(\mathrm{H} \beta)$ in $10^{-16} \mathrm{erg} \mathrm{s}^{-1} \mathrm{~cm}^{-2} \AA^{-1} ; \mathrm{EW}(\mathrm{H} \beta)$ in $\AA$; velocity in $\mathrm{km} \mathrm{s}^{-1}$. 
TABle 3

Continued

\begin{tabular}{|c|c|c|c|c|c|c|c|}
\hline \multirow[t]{2}{*}{ Line } & \multirow[t]{2}{*}{$f(\lambda)$} & \multicolumn{3}{|c|}{ KUG1603+179A } & \multicolumn{2}{|c|}{ NGC6050A } & \multirow[t]{2}{*}{ NGC6050B } \\
\hline & & $\mathrm{a}$ & $\mathrm{b}$ & $\mathrm{c}$ & $\mathrm{a}$ & $\mathrm{b}$ & \\
\hline $3727[$ O II] $\ldots \ldots \ldots$ & 0.271 & $205 \pm 5$ & $133 \pm 11$ & $172 \pm 7$ & $151 \pm 16$ & $128 \pm 15$ & $176 \pm 45$ \\
\hline $3869[\mathrm{Ne}$ III $] \ldots \ldots$ & 0.238 & $\ldots$ & $\ldots$ & $\ldots$ & $\ldots$ & $\ldots$ & $\ldots$ \\
\hline $3889 \mathrm{He} \mathrm{I}+\mathrm{H} 8 \ldots \ldots$ & 0.233 & $\ldots$ & $\cdots$ & $24 \pm 1$ & $\cdots$ & $35 \pm 2$ & $\cdots$ \\
\hline $3968[\mathrm{Ne}$ III $]+\mathrm{H} 7 \ldots$ & 0.216 & $17 \pm 3$ & $\cdots$ & $\ldots$ & $\cdots$ & $18 \pm 2$ & $\cdots$ \\
\hline $4069[\mathrm{~S}$ II $] \ldots \ldots \ldots$ & 0.195 & $14 \pm 2$ & $\cdots$ & $\ldots$ & $\cdots$ & $\ldots$ & $\ldots$ \\
\hline $4102 \mathrm{H} \delta \ldots \ldots \ldots$ & 0.188 & $25 \pm 4$ & $\ldots$ & $28 \pm 5$ & $\cdots$ & $27 \pm 8$ & $\ldots$ \\
\hline $4340 \mathrm{H} \gamma \ldots \ldots \ldots$ & 0.142 & $46 \pm 2$ & $41 \pm 5$ & $49 \pm 2$ & $\ldots$ & $43 \pm 8$ & $48 \pm 11$ \\
\hline $4861 \mathrm{H} \beta \ldots \ldots \ldots$ & 0.00 & $100 \pm 3$ & $100 \pm 3$ & $100 \pm 3$ & $100 \pm 10$ & $100 \pm 3$ & $100 \pm 5$ \\
\hline $4959[$ O III $] \ldots \ldots$ & -0.024 & $\ldots$ & $13 \pm 3$ & $17 \pm 3$ & $\ldots$ & $\ldots$ & $38 \pm 6$ \\
\hline $5007[\mathrm{O}$ III $] \ldots \ldots$ & -0.035 & $43 \pm 2$ & $37 \pm 4$ & $48 \pm 2$ & $61 \pm 5$ & $33 \pm 3$ & $107 \pm 6$ \\
\hline 5876 Не I......... & -0.209 & $12 \pm 2$ & $\ldots$ & $9 \pm 1$ & $\cdots$ & $\cdots$ & $\ldots$ \\
\hline $6300[\mathrm{O}$ I $] \ldots \ldots \ldots$ & -0.276 & $8 \pm 1$ & $16 \pm 2$ & $\ldots$ & $41 \pm 6$ & $5 \pm 1$ & $\cdots$ \\
\hline $6548[\mathrm{~N}$ II $] \ldots \ldots \ldots$ & -0.311 & $36 \pm 2$ & $44 \pm 5$ & $40 \pm 1$ & $53 \pm 8$ & $34 \pm 3$ & $\ldots$ \\
\hline $6563 \mathrm{H} \alpha \ldots \ldots \ldots$ & -0.313 & $279 \pm 4$ & $261 \pm 23$ & $306 \pm 10$ & $286 \pm 38$ & $285 \pm 15$ & $293 \pm 9$ \\
\hline $6584[\mathrm{~N}$ II $] \ldots \ldots \ldots$ & -0.316 & $105 \pm 2$ & $148 \pm 13$ & $116 \pm 4$ & $184 \pm 23$ & $100 \pm 6$ & $82 \pm 7$ \\
\hline $6717[\mathrm{~S}$ II $] \ldots \ldots \ldots$ & -0.334 & $46 \pm 1$ & $36 \pm 4$ & $47 \pm 2$ & $36 \pm 7$ & $37 \pm 3$ & $45 \pm 4$ \\
\hline $6731[\mathrm{~S} \mathrm{II}] \ldots \ldots \ldots$ & -0.336 & $39 \pm 1$ & $47 \pm 5$ & $38 \pm 2$ & $69 \pm 11$ & $36 \pm 3$ & $65 \pm 10$ \\
\hline $\mathrm{F}(\mathrm{H} \beta)$ & & $11.2 \pm 0.4$ & $16.0 \pm 0.5$ & $14.4 \pm 0.5$ & $8.0 \pm 0.8$ & $10.2 \pm 0.4$ & $7.7 \pm 0.4$ \\
\hline $\operatorname{EW}(\mathrm{H} \beta)$ & & $9.3 \pm 0.7$ & $4.9 \pm 0.3$ & $10.4 \pm 0.7$ & $2.6 \pm 0.3$ & $15.1 \pm 1.5$ & $4.9 \pm 0.5$ \\
\hline $\mathrm{c}(\mathrm{H} \beta)$ & & $0.49 \pm 0.02$ & $1.25 \pm 0.12$ & $0.35 \pm 0.05$ & $0.32 \pm 0.17$ & $0.57 \pm 0.07$ & $0.16 \pm 0.03$ \\
\hline velocity & & $11280 \pm 30$ & $11162 \pm 20$ & $11121 \pm 17$ & $9524 \pm 38$ & $9686 \pm 37$ & $11134 \pm 39$ \\
\hline
\end{tabular}

TABle 3

Continued

\begin{tabular}{|c|c|c|c|c|c|c|c|}
\hline \multirow[t]{2}{*}{ Line } & \multirow[t]{2}{*}{$f(\lambda)$} & \multirow[t]{2}{*}{ LEDA1543586 } & \multicolumn{5}{|c|}{ NGC6045 } \\
\hline & & & $\mathrm{a}$ & $\mathrm{b}$ & c & $\mathrm{d}$ & $\mathrm{e}$ \\
\hline $3727[\mathrm{O}$ II $] \ldots \ldots \ldots$ & 0.271 & $281 \pm 4$ & $334 \pm 21$ & $214 \pm 8$ & $141 \pm 12$ & $152 \pm 9$ & $310 \pm 5$ \\
\hline $3869[\mathrm{Ne} \mathrm{III}] \ldots . .$. & 0.238 & $\ldots$ & $\ldots$ & $\ldots$ & $\ldots$ & $\ldots$ & $\ldots$ \\
\hline $3889 \mathrm{He} \mathrm{I}+\mathrm{H} 8 \ldots \ldots$ & 0.233 & $\ldots$ & $\ldots$ & $\ldots$ & $\ldots$ & $\ldots$ & $\ldots$ \\
\hline $3968[\mathrm{Ne}$ III $]+\mathrm{H} 7 \ldots$ & 0.216 & $\ldots$ & $\cdots$ & $\ldots$ & $\ldots$ & $19 \pm 4$ & $\ldots$ \\
\hline $4069[\mathrm{~S} \mathrm{II}] \ldots \ldots$ & 0.195 & $\ldots$ & $\ldots$ & $\ldots$ & $\ldots$ & $\ldots$ & $\ldots$ \\
\hline $4102 \mathrm{H} \delta \ldots \ldots \ldots$ & 0.188 & $\ldots$ & $\ldots$ & $\ldots$ & $\ldots$ & $25 \pm 6$ & $\ldots$ \\
\hline $4340 \mathrm{H} \gamma \ldots \ldots \ldots$ & 0.142 & $46 \pm 8$ & $45 \pm 6$ & $47 \pm 5$ & $\ldots$ & $49 \pm 3$ & $48 \pm 9$ \\
\hline $4861 \mathrm{H} \beta \ldots \ldots \ldots$ & 0.00 & $100 \pm 2$ & $100 \pm 8$ & $100 \pm 1$ & $100 \pm 6$ & $100 \pm 4$ & $100 \pm 5$ \\
\hline $4959[\mathrm{O}$ III $] \ldots \ldots$ & -0.024 & $103 \pm 4$ & $\ldots$ & $\ldots$ & $\ldots$ & $\ldots$ & $20 \pm 4$ \\
\hline $5007[\mathrm{O}$ III $] \ldots \ldots$ & -0.035 & $310 \pm 4$ & $40 \pm 8$ & $35 \pm 3$ & $44 \pm 2$ & $19 \pm 1$ & $65 \pm 3$ \\
\hline 5876 He I.......... & -0.209 & $\ldots$ & $\ldots$ & $\ldots$ & $\ldots$ & $8 \pm 1$ & $8 \pm 1$ \\
\hline $6300[\mathrm{O}$ I $] \ldots \ldots \ldots$ & -0.276 & $22 \pm 2$ & $\ldots$ & $\ldots$ & $\cdots$ & $14 \pm 2$ & $23 \pm 1$ \\
\hline $6548[\mathrm{~N} \mathrm{II}] \ldots \ldots \ldots$ & -0.311 & $17 \pm 4$ & $44 \pm 5$ & $42 \pm 1$ & $\ldots$ & $31 \pm 1$ & $37 \pm 2$ \\
\hline $6563 \mathrm{H} \alpha \ldots \ldots \ldots$ & -0.313 & $286 \pm 4$ & $280 \pm 7$ & $288 \pm 2$ & $287 \pm 20$ & $288 \pm 10$ & $292 \pm 6$ \\
\hline $6584[\mathrm{~N} \mathrm{II}] \ldots \ldots \ldots$ & -0.316 & $44 \pm 5$ & $118 \pm 6$ & $109 \pm 3$ & $203 \pm 16$ & $92 \pm 3$ & $110 \pm 3$ \\
\hline $6717[\mathrm{~S} \mathrm{II}] \ldots \ldots \ldots$ & -0.334 & $46 \pm 2$ & $46 \pm 3$ & $37 \pm 2$ & $38 \pm 4$ & $28 \pm 1$ & $51 \pm 1$ \\
\hline $6731[\mathrm{~S} \mathrm{II}] \ldots \ldots \ldots$ & -0.336 & $29 \pm 2$ & $44 \pm 4$ & $27 \pm 3$ & $44 \pm 3$ & $22 \pm 1$ & $38 \pm 1$ \\
\hline $\mathrm{F}(\mathrm{H} \beta)$ & & $6.9 \pm 0.1$ & $5.2 \pm 0.4$ & $8.5 \pm 0.1$ & $5.4 \pm 0.3$ & $17.2 \pm 0.7$ & $15.4 \pm 0.7$ \\
\hline $\mathrm{EW}(\mathrm{H} \beta)$ & & $8.9 \pm 1.0$ & $3.0 \pm 0.3$ & $3.3 \pm 0.1$ & $2.1 \pm 0.2$ & $7.9 \pm 0.4$ & $5.7 \pm 0.4$ \\
\hline $\mathrm{c}(\mathrm{H} \beta)$ & & $0.17 \pm 0.01$ & $1.05 \pm 0.03$ & $1.04 \pm 0.01$ & $0.18 \pm 0.09$ & $0.96 \pm 0.05$ & $0.94 \pm 0.02$ \\
\hline velocity & & $9849 \pm 13$ & $10284 \pm 11$ & $10335 \pm 12$ & $9830 \pm 71$ & $9703 \pm 15$ & $9789 \pm 23$ \\
\hline
\end{tabular}


TABle 3

Continued

\begin{tabular}{|c|c|c|c|c|c|c|c|c|}
\hline \multirow[t]{2}{*}{ Line } & \multirow[t]{2}{*}{$f(\lambda)$} & \multirow[t]{2}{*}{ KUG1602+174A } & \multirow[t]{2}{*}{ LEDA084719 } & \multicolumn{2}{|c|}{ PGC057077 } & \multicolumn{3}{|c|}{ UGC10190 } \\
\hline & & & & $\mathrm{a}$ & $\mathrm{b}$ & $\mathrm{a}$ & $\mathrm{b}$ & $\mathrm{c}$ \\
\hline $3727[\mathrm{O} \quad \mathrm{II}] \ldots$ & 0.271 & $175 \pm 10$ & $318 \pm 97$ & $328 \pm 17$ & $165 \pm 3$ & $241 \pm 36$ & $182 \pm 18$ & $230 \pm 52$ \\
\hline 3869 [Ne III] . . & 0.238 & $\ldots$ & $\ldots$ & $\ldots$ & $\ldots$ & $\ldots$ & $\ldots$ & $\ldots$ \\
\hline $3889 \mathrm{He} \mathrm{I}+\mathrm{H} 8 \ldots \ldots$ & 0.233 & $55 \pm 8$ & $\ldots$ & $\ldots$ & $13 \pm 1$ & $\ldots$ & $\ldots$ & $\ldots$ \\
\hline $3968[\mathrm{Ne}$ III $]+\mathrm{H} 7 \ldots$ & 0.216 & $\ldots$ & $\ldots$ & $\ldots$ & $23 \pm 2$ & $\ldots$ & $\ldots$ & $\ldots$ \\
\hline $4069[\mathrm{~S}$ II $] \ldots \ldots \ldots$ & 0.195 & $\ldots$ & $\ldots$ & $\ldots$ & $\ldots$ & $\ldots$ & $\ldots$ & $\ldots$ \\
\hline $4102 \mathrm{H} \delta \ldots \ldots \ldots$ & 0.188 & $\ldots$ & $\ldots$ & $26 \pm 4$ & $25 \pm 2$ & $\ldots$ & $\ldots$ & $\ldots$ \\
\hline $4340 \mathrm{H} \gamma \ldots \ldots \ldots$ & 0.142 & $43 \pm 5$ & $\ldots$ & $42 \pm 2$ & $45 \pm 1$ & $\ldots$ & $43 \pm 8$ & $46 \pm 18$ \\
\hline $4861 \mathrm{H} \beta \ldots \ldots \ldots$ & 0.00 & $100 \pm 5$ & $100 \pm 8$ & $100 \pm 5$ & $100 \pm 1$ & $100 \pm 6$ & $100 \pm 7$ & $100 \pm 10$ \\
\hline $4959[$ O III $] \ldots \ldots \ldots$ & -0.024 & $\ldots$ & $48 \pm 11$ & $\ldots$ & $20 \pm 1$ & $\ldots$ & $\ldots$ & $24 \pm 3$ \\
\hline $5007[$ O III $] \ldots \ldots \ldots$ & -0.035 & $25 \pm 7$ & $\ldots$ & $63 \pm 3$ & $55 \pm 1$ & $36 \pm 2$ & $24 \pm 3$ & $79 \pm 3$ \\
\hline $5876 \mathrm{He}$ I......... & -0.209 & $\ldots$ & $\ldots$ & $12 \pm 2$ & $13 \pm 1$ & $\ldots$ & $\ldots$ & $\ldots$ \\
\hline $6300[\mathrm{O}$ I $] \ldots \ldots \ldots$ & -0.276 & $14 \pm 2$ & $\ldots$ & $14 \pm 3$ & $6 \pm 1$ & $\ldots$ & $\ldots$ & $\cdots$ \\
\hline $6548[\mathrm{~N}$ II $] \ldots \ldots \ldots$ & -0.311 & $31 \pm 3$ & $46 \pm 7$ & $36 \pm 2$ & $33 \pm 1$ & $35 \pm 11$ & $32 \pm 4$ & $\ldots$ \\
\hline $6563 \mathrm{H} \alpha \ldots \ldots \ldots$ & -0.313 & $272 \pm 14$ & $286 \pm 23$ & $277 \pm 13$ & $277 \pm 5$ & $286 \pm 18$ & $272 \pm 14$ & $282 \pm 7$ \\
\hline $6584[\mathrm{~N}$ II $] \ldots \ldots \ldots$ & -0.316 & $99 \pm 6$ & $112 \pm 12$ & $103 \pm 5$ & $93 \pm 2$ & $111 \pm 8$ & $98 \pm 7$ & $73 \pm 6$ \\
\hline $6717[\mathrm{~S} \mathrm{HI}] \ldots \ldots \ldots$ & -0.334 & $49 \pm 4$ & $42 \pm 5$ & $38 \pm 2$ & $34 \pm 1$ & $64 \pm 5$ & $60 \pm 5$ & $90 \pm 8$ \\
\hline $6731[\mathrm{~S}$ II] $\ldots \ldots \ldots$ & -0.336 & $41 \pm 5$ & $40 \pm 7$ & $31 \pm 2$ & $27 \pm 1$ & $83 \pm 7$ & $38 \pm 3$ & $64 \pm 5$ \\
\hline $\mathrm{F}(\mathrm{H} \beta)$ & & $5.9 \pm 0.3$ & $2.7 \pm 0.2$ & $5.9 \pm 0.3$ & $52.0 \pm 0.5$ & $1.4 \pm 0.1$ & $7.2 \pm 0.5$ & $1.9 \pm 0.2$ \\
\hline $\operatorname{EW}(\mathrm{H} \beta)$ & & $4.1 \pm 0.3$ & $1.7 \pm 0.2$ & $7.8 \pm 0.6$ & $17.3 \pm 0.7$ & $3.5 \pm 0.4$ & $4.1 \pm 0.3$ & $6.8 \pm 1.2$ \\
\hline $\mathrm{c}(\mathrm{H} \beta)$ & & $0.54 \pm 0.07$ & $1.24 \pm 0.11$ & $2.14 \pm 0.07$ & $0.13 \pm 0.02$ & $0.55 \pm 0.09$ & $0.61 \pm 0.07$ & $0.08 \pm 0.02$ \\
\hline velocity & & $10614 \pm 18$ & $10155 \pm 14$ & $10209 \pm 15$ & $10200 \pm 14$ & $11171 \pm 30$ & $11046 \pm 13$ & $10915 \pm 25$ \\
\hline
\end{tabular}

TABle 3

Continued

\begin{tabular}{|c|c|c|c|c|c|c|c|}
\hline \multirow[t]{2}{*}{ Line } & \multirow[t]{2}{*}{$f(\lambda)$} & \multirow[t]{2}{*}{ LEDA140568 } & \multirow[t]{2}{*}[D97]{$c e-200$} & \multicolumn{2}{|c|}{ PGC057064 } & \multicolumn{2}{|c|}{ LEDA084703 } \\
\hline & & & & $\mathrm{a}$ & $\mathrm{b}$ & int & $\mathrm{a}$ \\
\hline $3727[$ O & 0.271 & $314 \pm 20$ & $326 \pm 23$ & $256 \pm 23$ & $636 \pm 21$ & $331 \pm 5$ & $339 \pm 10$ \\
\hline $3869[\mathrm{Ne} \mathrm{III}] \ldots \ldots$ & 0.238 & $44 \pm 9$ & $\ldots$ & $\ldots$ & $\ldots$ & $22 \pm 1$ & $22 \pm 2$ \\
\hline $3889 \mathrm{He} \mathrm{I}+\mathrm{H} 8 \ldots \ldots$ & 0.233 & $\ldots$ & $\cdots$ & $\cdots$ & $\cdots$ & $22 \pm 1$ & $15 \pm 2$ \\
\hline $3968[\mathrm{Ne}$ III $]+\mathrm{H} 7 \ldots$ & 0.216 & $\cdots$ & $\cdots$ & $\cdots$ & $\ldots$ & $18 \pm 1$ & $20 \pm 3$ \\
\hline $4069[\mathrm{~S} \mathrm{II}] \ldots \ldots \ldots$ & 0.195 & $\ldots$ & $\cdots$ & $\cdots$ & $44 \pm 8$ & $\ldots$ & $\ldots$ \\
\hline $4102 \mathrm{H} \delta \ldots \ldots \ldots$ & 0.188 & $\ldots$ & $\ldots$ & $\ldots$ & $\ldots$ & $26 \pm 2$ & $27 \pm 2$ \\
\hline $4340 \mathrm{H} \gamma \ldots \ldots \ldots$ & 0.142 & $47 \pm 10$ & $46 \pm 3$ & $\ldots$ & $46 \pm 8$ & $49 \pm 1$ & $49 \pm 1$ \\
\hline $4861 \mathrm{H} \beta \ldots \ldots \ldots$ & 0.00 & $100 \pm 6$ & $100 \pm 8$ & $100 \pm 7$ & $100 \pm 5$ & $100 \pm 1$ & $100 \pm 1$ \\
\hline $4959[$ O III $] \ldots \ldots$ & -0.024 & $70 \pm 4$ & $53 \pm 6$ & $\ldots$ & $41 \pm 10$ & $57 \pm 3$ & $61 \pm 2$ \\
\hline 5007 [O III $] \ldots \ldots$ & -0.035 & $214 \pm 5$ & $163 \pm 4$ & $45 \pm 4$ & $134 \pm 8$ & $177 \pm 1$ & $187 \pm 2$ \\
\hline 5876 He I........ & -0.209 & $\ldots$ & $\ldots$ & $\ldots$ & $\ldots$ & $14 \pm 1$ & $14 \pm 2$ \\
\hline $6300[\mathrm{O}$ I $] \ldots \ldots \ldots$ & -0.276 & $\cdots$ & $20 \pm 1$ & $13 \pm 4$ & $16 \pm 2$ & $14 \pm 1$ & $12 \pm 1$ \\
\hline $6548[\mathrm{~N}$ II $] \ldots \ldots \ldots$ & -0.311 & $\ldots$ & $12 \pm 3$ & $44 \pm 4$ & $56 \pm 3$ & $16 \pm 2$ & $11 \pm 2$ \\
\hline $6563 \mathrm{H} \alpha \ldots \ldots \ldots$ & -0.313 & $286 \pm 20$ & $286 \pm 2$ & $286 \pm 19$ & $282 \pm 6$ & $292 \pm 6$ & $219^{\dagger}$ \\
\hline $6584[\mathrm{~N}$ II $] \ldots \ldots \ldots$ & -0.316 & $16 \pm 5$ & $35 \pm 2$ & $126 \pm 10$ & $155 \pm 5$ & $43 \pm 2$ & $31 \pm 1$ \\
\hline $6717[\mathrm{~S} \mathrm{II}] \ldots \ldots \ldots$ & -0.334 & $39 \pm 3$ & $60 \pm 1$ & $48 \pm 4$ & $77 \pm 5$ & $55 \pm 2$ & $39 \pm 2$ \\
\hline $6731[\mathrm{~S}$ II $] \ldots \ldots \ldots$ & -0.336 & $23 \pm 3$ & $51 \pm 1$ & $27 \pm 2$ & $64 \pm 6$ & $40 \pm 2$ & $29 \pm 1$ \\
\hline $\mathrm{F}(\mathrm{H} \beta)$ & & $4.1 \pm 0.3$ & $3.8 \pm 0.3$ & $6.0 \pm 0.4$ & $5.5 \pm 0.3$ & $30.2 \pm 0.2$ & $9.2 \pm 0.1$ \\
\hline $\mathrm{EW}(\mathrm{H} \beta)$ & & $10.4 \pm 10.1$ & $9.7 \pm 1.4$ & $2.9 \pm 0.2$ & $1.7 \pm 0.1$ & $14.4 \pm 0.8$ & $17.1 \pm 1.5$ \\
\hline $\mathrm{c}(\mathrm{H} \beta)$ & & $-0.03 \pm 0.09$ & $0.10 \pm 0.01$ & $0.96 \pm 0.09$ & $1.04 \pm 0.02$ & $0.25 \pm 0.03$ & $0.00 \pm 0.05$ \\
\hline velocity & & $11992 \pm 25$ & $9915 \pm 8$ & $10308 \pm 24$ & $10048 \pm 21$ & $10033 \pm 6$ & $10008 \pm 4$ \\
\hline
\end{tabular}

${ }^{\dagger}$ Uncertain value. 
TABle 3

Continued

\begin{tabular}{|c|c|c|c|c|c|c|c|}
\hline \multirow[t]{2}{*}{ Line } & \multirow[t]{2}{*}{$f(\lambda)$} & \multicolumn{2}{|c|}{ LEDA084703 } & \multirow[t]{2}{*}{ KUG1602+175 } & \multirow[t]{2}{*}{ LEDA084710 } & \multirow[t]{2}{*}{ CGCG108-149 } & \multirow[t]{2}{*}{ KUG1602+174B } \\
\hline & & $\mathrm{b}$ & $\mathrm{c}$ & & & & \\
\hline $3727[\mathrm{O} \quad \mathrm{II}] \ldots$ & 0.271 & $360 \pm 8$ & $514 \pm 10$ & $159 \pm 12$ & $361 \pm 110$ & $159 \pm 53$ & $164 \pm 18$ \\
\hline $3869[\mathrm{Ne} \mathrm{III}] \ldots$ & 0.238 & $21 \pm 2$ & $\ldots$ & $\ldots$ & $\ldots$ & $\ldots$ & $\ldots$ \\
\hline $3889 \mathrm{He} \mathrm{I}+\mathrm{H} 8 \ldots \ldots$ & 0.233 & $12 \pm 7$ & $\ldots$ & $\ldots$ & $\ldots$ & $\ldots$ & $\ldots$ \\
\hline $3968[\mathrm{Ne}$ III $]+\mathrm{H} 7 \ldots$ & 0.216 & $\ldots$ & $\cdots$ & $22 \pm 4$ & $\cdots$ & $\cdots$ & $\cdots$ \\
\hline $4069[\mathrm{~S}$ II $] \ldots \ldots \ldots$ & 0.195 & $\ldots$ & $\cdots$ & $\ldots$ & $\cdots$ & $\cdots$ & $\cdots$ \\
\hline $4102 \mathrm{H} \delta \ldots \ldots \ldots$ & 0.188 & $24 \pm 1$ & $\ldots$ & $25 \pm 3$ & $\ldots$ & $\cdots$ & $\ldots$ \\
\hline $4340 \mathrm{H} \gamma \ldots \ldots \ldots$ & 0.142 & $47 \pm 1$ & $47 \pm 8$ & $47 \pm 5$ & $\ldots$ & $\ldots$ & $46 \pm 7$ \\
\hline $4861 \mathrm{H} \beta \ldots \ldots \ldots$ & 0.00 & $100 \pm 1$ & $100 \pm 2$ & $100 \pm 8$ & $100 \pm 11$ & $100 \pm 17$ & $100 \pm 6$ \\
\hline 4959 [O III $] \ldots \ldots$ & -0.024 & $57 \pm 1$ & $44 \pm 2$ & $\ldots$ & $\ldots$ & $\ldots$ & $\ldots$ \\
\hline $5007[$ O III $] \ldots \ldots$. & -0.035 & $186 \pm 2$ & $131 \pm 3$ & $56 \pm 7$ & $82 \pm 9$ & $53 \pm 4$ & $50 \pm 5$ \\
\hline 5876 He I......... & -0.209 & $13 \pm 2$ & $11 \pm 1$ & $\ldots$ & $7 \pm 4$ & $\ldots$ & $\ldots$ \\
\hline $6300[\mathrm{O}$ I $] \ldots \ldots \ldots$ & -0.276 & $13 \pm 1$ & $20 \pm 5$ & $\ldots$ & $15 \pm 4$ & $\cdots$ & $\ldots$ \\
\hline $6548[\mathrm{~N}$ II $] \ldots \ldots \ldots$ & -0.311 & $14 \pm 1$ & $20 \pm 1$ & $37 \pm 2$ & $30 \pm 5$ & $\ldots$ & $34 \pm 6$ \\
\hline $6563 \mathrm{H} \alpha \ldots \ldots \ldots$ & -0.313 & $280 \pm 7$ & $290 \pm 3$ & $282 \pm 7$ & $285 \pm 34$ & $283 \pm 52$ & $283 \pm 6$ \\
\hline $6584[\mathrm{~N}$ II $] \ldots \ldots \ldots$ & -0.316 & $42 \pm 1$ & $49 \pm 2$ & $103 \pm 6$ & $89 \pm 12$ & $165 \pm 32$ & $97 \pm 5$ \\
\hline $6717[\mathrm{~S}$ II $] \ldots \ldots \ldots$ & -0.334 & $48 \pm 1$ & $84 \pm 2$ & $45 \pm 3$ & $63 \pm 9$ & $50 \pm 14$ & $55 \pm 4$ \\
\hline $6731[\mathrm{~S} \mathrm{II}] \ldots \ldots \ldots$ & -0.336 & $38 \pm 1$ & $48 \pm 2$ & $23 \pm 4$ & $36 \pm 6$ & $54 \pm 15$ & $44 \pm 4$ \\
\hline $\mathrm{F}(\mathrm{H} \beta)$ & & $17.0 \pm 0.0$ & $3.7 \pm 0.1$ & $27.2 \pm 2.1$ & $10.0 \pm 1.1$ & $4.1 \pm 0.7$ & $11.6 \pm 0.7$ \\
\hline $\mathrm{EW}(\mathrm{H} \beta)$ & & $16.5 \pm 0.8$ & $6.2 \pm 0.6$ & $4.2 \pm 0.4$ & $3.3 \pm 0.5$ & $1.4 \pm 0.2$ & $4.8 \pm 0.5$ \\
\hline $\mathrm{c}(\mathrm{H} \beta)$ & & $0.59 \pm 0.03$ & $0.50 \pm 0.01$ & $0.45 \pm 0.03$ & $1.18 \pm 0.16$ & $1.16 \pm 0.25$ & $0.48 \pm 0.02$ \\
\hline velocity & & $10032 \pm 11$ & $10087 \pm 16$ & $10574 \pm 20$ & $10781 \pm 32$ & $11121 \pm 15$ & $10622 \pm 15$ \\
\hline
\end{tabular}

TABle 3

Continued

\begin{tabular}{|c|c|c|c|c|c|c|}
\hline Line & $f(\lambda)$ & LEDA084724 & SDSS J160556.98 & [DKP87]160310.21 & SDSS J160524.27 & SDSS J160523.66 \\
\hline $3727\left[\begin{array}{ll}\mathrm{O} & \mathrm{II}\end{array}\right] \ldots \ldots \ldots$ & 0.271 & $493 \pm 14$ & $317 \pm 19$ & $281 \pm 13$ & $214 \pm 13$ & $405 \pm 5$ \\
\hline $3869[\mathrm{Ne} \mathrm{III}] \ldots \ldots$ & 0.238 & $\ldots$ & $21 \pm 6$ & $\ldots$ & $30 \pm 3$ & $\ldots$ \\
\hline $3889 \mathrm{He} \mathrm{I}+\mathrm{H} 8 \ldots \ldots$ & 0.233 & $\ldots$ & $19 \pm 6$ & $\ldots$ & $16 \pm 2$ & $\ldots$ \\
\hline $3968[\mathrm{Ne}$ III $]+\mathrm{H} 7 \ldots$ & 0.216 & $\ldots$ & $36 \pm 5$ & $\ldots$ & $28 \pm 2$ & $\ldots$ \\
\hline $4069[\mathrm{~S}$ II $] \ldots \ldots \ldots$ & 0.195 & $\cdots$ & $\ldots$ & $\cdots$ & $\ldots$ & $\cdots$ \\
\hline $4102 \mathrm{H} \delta \ldots \ldots \ldots$ & 0.188 & $\ldots$ & $25 \pm 3$ & $\ldots$ & $30 \pm 3$ & $\ldots$ \\
\hline $4340 \mathrm{H} \gamma \ldots \ldots \ldots$ & 0.142 & $49 \pm 6$ & $47 \pm 4$ & $46 \pm 5$ & $49 \pm 5$ & $47 \pm 3$ \\
\hline $4861 \mathrm{H} \beta \ldots \ldots \ldots$ & 0.00 & $100 \pm 8$ & $100 \pm 6$ & $100 \pm 8$ & $100 \pm 3$ & $100 \pm 4$ \\
\hline 4959 [O III $] \ldots \ldots$ & -0.024 & $\ldots$ & $95 \pm 4$ & $\cdots$ & $114 \pm 3$ & $\ldots$ \\
\hline 5007 [O III $] \ldots \ldots$ & -0.035 & $91 \pm 5$ & $282 \pm 4$ & $47 \pm 3$ & $337 \pm 4$ & $69 \pm 8$ \\
\hline 5876 Не I......... & -0.209 & $\ldots$ & $10 \pm 1$ & $\ldots$ & $13 \pm 2$ & $\ldots$ \\
\hline $6300[\mathrm{O}$ I $] \ldots \ldots \ldots$ & -0.276 & $\cdots$ & $16 \pm 4$ & $\ldots$ & $10 \pm 1$ & $\ldots$ \\
\hline $6548\left[\mathrm{~N}_{\mathrm{II}}\right] \ldots \ldots \ldots$ & -0.311 & $\ldots$ & $\ldots$ & $27 \pm 2$ & $\ldots$ & $29 \pm 8$ \\
\hline $6563 \mathrm{H} \alpha \ldots \ldots \ldots$ & -0.313 & $296 \pm 13$ & $280 \pm 7$ & $284 \pm 4$ & $320 \pm 21$ & $289 \pm 2$ \\
\hline $6584[\mathrm{~N}$ II $] \ldots \ldots \ldots$ & -0.316 & $62 \pm 8$ & $27 \pm 6$ & $75 \pm 3$ & $13 \pm 2$ & $61 \pm 2$ \\
\hline $6717[\mathrm{~S}$ II $] \ldots \ldots \ldots$ & -0.334 & $74 \pm 8$ & $40 \pm 2$ & $72 \pm 2$ & $35 \pm 3$ & $72 \pm 3$ \\
\hline $6731[\mathrm{~S}$ III $] \ldots \ldots \ldots$ & -0.336 & $50 \pm 6$ & $27 \pm 2$ & $66 \pm 4$ & $22 \pm 2$ & $64 \pm 3$ \\
\hline $\mathrm{F}(\mathrm{H} \beta)$ & & $3.8 \pm 0.3$ & $9.6 \pm 0.5$ & $3.6 \pm 0.3$ & $6.6 \pm 0.2$ & $2.0 \pm 0.1$ \\
\hline $\mathrm{EW}(\mathrm{H} \beta)$ & & $2.7 \pm 0.4$ & $18.1 \pm 2.3$ & $4.4 \pm 0.5$ & $19.3 \pm 1.9$ & $3.6 \pm 0.4$ \\
\hline $\mathrm{c}(\mathrm{H} \beta)$ & & $0.43 \pm 0.04$ & $0.57 \pm 0.03$ & $0.09 \pm 0.01$ & $0.03 \pm 0.09$ & $0.25 \pm 0.01$ \\
\hline velocity & & $11714 \pm 60$ & $11621 \pm 24$ & $10308 \pm 56$ & $11101 \pm 38$ & $10337 \pm 35$ \\
\hline
\end{tabular}


TABLE 3

Continued

\begin{tabular}{|c|c|c|c|c|c|c|}
\hline Line & $f(\lambda)$ & IC1182 & IC1182:[S72]d & SDSS J150531.84 & SDSS J160304.20 & SDSS J160520.58 \\
\hline $3727[\mathrm{O}$ ІІ $] \ldots \ldots \ldots$ & 0.271 & $379 \pm 22$ & $399 \pm 9$ & $74 \pm 4$ & $455 \pm 26$ & $108 \pm 30$ \\
\hline $3869[$ Ne III $] \ldots . .$. & 0.238 & $23 \pm 2$ & $35 \pm 1$ & $\ldots$ & $\ldots$ & $\ldots$ \\
\hline $3889 \mathrm{He} \mathrm{I}+\mathrm{H} 8 \ldots \ldots$ & 0.233 & $21 \pm 2$ & $20 \pm 2$ & $\ldots$ & $\ldots$ & $\ldots$ \\
\hline $3968[\mathrm{Ne}$ III $]+\mathrm{H} 7 \ldots$ & 0.216 & $24 \pm 3$ & $24 \pm 3$ & $\cdots$ & $\ldots$ & $\ldots$ \\
\hline $4069[\mathrm{~S} \mathrm{II}] \ldots \ldots \ldots$ & 0.195 & $\ldots$ & $\ldots$ & $\ldots$ & $\ldots$ & $\ldots$ \\
\hline $4102 \mathrm{H} \delta \ldots \ldots \ldots$ & 0.188 & $30 \pm 2$ & $27 \pm 1$ & $\cdots$ & $\ldots$ & $\ldots$ \\
\hline $4340 \mathrm{H} \gamma \ldots \ldots \ldots$ & 0.142 & $48 \pm 2$ & $47 \pm 3$ & $\ldots$ & $44 \pm 3$ & $46 \pm 4$ \\
\hline $4861 \mathrm{H} \beta \ldots \ldots \ldots$ & 0.00 & $100 \pm 2$ & $100 \pm 2$ & $100 \pm 5$ & $100 \pm 2$ & $100 \pm 10$ \\
\hline 4959 [O III $] \ldots \ldots$. & -0.024 & $73 \pm 2$ & $87 \pm 1$ & $\ldots$ & $\ldots$ & $\ldots$ \\
\hline $5007[$ O III $] \ldots \ldots \ldots$ & -0.035 & $218 \pm 4$ & $253 \pm 2$ & $96 \pm 3$ & $66 \pm 4$ & $11 \pm 3$ \\
\hline 5876 Не I......... & -0.209 & $11 \pm 1$ & $\ldots$ & $\ldots$ & $\ldots$ & $\ldots$ \\
\hline $6300[\mathrm{O}$ I $] \ldots \ldots \ldots$ & -0.276 & $36 \pm 3$ & $14 \pm 1$ & $18 \pm 2$ & $11 \pm 1$ & $\ldots$ \\
\hline 6548 [N II $] \ldots \ldots \ldots$ & -0.311 & $\ldots$ & $13 \pm 1$ & $\ldots$ & $24 \pm 2$ & $35 \pm 2$ \\
\hline $6563 \mathrm{H} \alpha \ldots \ldots \ldots$ & -0.313 & $317 \pm 21$ & $298 \pm 7$ & $287 \pm 15$ & $272 \pm 13$ & $286 \pm 2$ \\
\hline $6584[\mathrm{~N}$ II $] \ldots \ldots \ldots$ & -0.316 & $83 \pm 8$ & $36 \pm 1$ & $87 \pm 5$ & $73 \pm 4$ & $99 \pm 2$ \\
\hline $6717[\mathrm{~S} \mathrm{II}] \ldots \ldots \ldots$ & -0.334 & $78 \pm 7$ & $48 \pm 1$ & $27 \pm 8$ & $65 \pm 4$ & $33 \pm 2$ \\
\hline $6731[\mathrm{~S}$ II $] \ldots \ldots \ldots$ & -0.336 & $73 \pm 10$ & $31 \pm 1$ & $14 \pm 2$ & $48 \pm 3$ & $33 \pm 2$ \\
\hline $\mathrm{F}(\mathrm{H} \beta)$ & & $167.5 \pm 3.5$ & $13.1 \pm 0.2$ & $1.9 \pm 0.1$ & $6.3 \pm 0.1$ & $3.0 \pm 0.3$ \\
\hline $\mathrm{EW}(\mathrm{H} \beta)$ & & $28.5 \pm 0.9$ & $29.7 \pm 4.6$ & $4.3 \pm 0.5$ & $0.0 \pm 0.0$ & $0.0 \pm 0.0$ \\
\hline $\mathrm{c}(\mathrm{H} \beta)$ & & $0.68 \pm 0.09$ & $0.37 \pm 0.03$ & $0.64 \pm 0.07$ & $0.76 \pm 0.07$ & $1.04 \pm 0.01$ \\
\hline velocity & & $10191 \pm 43$ & $10186 \pm 4$ & $9485 \pm 2$ & $10830 \pm 24$ & $10320 \pm 36$ \\
\hline
\end{tabular}

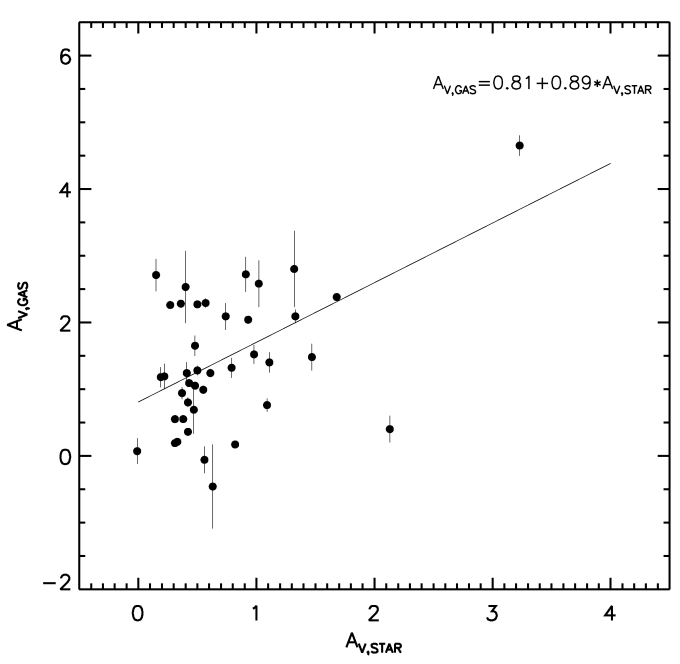

Fig. 4.- The gas-phase extinction estimated using the Balmer emission lines versus the extinction of the stellar component derived by STARLIGHT model fits on the continuum emission. A weighted linear fit to the data (solid line) yields the relation $A_{V, \text { gas }}=0.81+0.89 A_{V, \text { star }}$. ies for which we have ORM long-slit spectrum. We have checked first whether the SDSS aperture position matches the long-slit locus, and then we have extracted the spectrum from the corresponding area of the long-slit. The comparison is shown in Fig. 5 where the principal emission lines are shown when measured, without applying any reddening correction to them (cyan: [O II] 3727, red: $\mathrm{H} \alpha$ 6563, blue: [O III] 5007, pink: [N II] 6583, green: [S II] 6717 and yellow: [S II] 6731). Despite the fact that the covered regions are not identical (this could be a problem because of the inhomogeneous nature of star-forming regions), we can see that line ratios show a good agreement. While most of the points lie within the $0.2 \mathrm{dex}$ scatter region from the 1:1 line, there are some emission features that display larger discrepancy. This mostly happens in the cases of galaxies that SDSS aperture is not completely coincident with our long-slit spectrum.

In addition, IP03 have spectroscopic data for 7 galaxies belonging to $\mathrm{C} 09$ sample of SF galaxies in the Hercules cluster. We have compared the line fluxes, before the reddening correction, for 5 galaxies for which we have spectra in common (Fig. 5 open circles). We also see a very good agreement, with a small variation expected given the different position angles and different data analyzes.

The galaxies SDSSJ160304.20+171126.7, SDSSJ160 
$520.58+175210.6$ and SDSSJ160305.24+171136.1, quoted in the C09 sample, only have SDSS spectroscopy. The same procedure described in 93 and 4.1 has been applied to these galaxies. For the latter, even after applying STARLIGHT on the SDSS spectrum, we do not appreciate any emission line, because the SDSS fiber is placed in the center of the galaxy where no $\mathrm{H} \alpha$ emission is present. The other two are included in our sample and their STARLIGHT outputs and line flux measurements are also quoted in Table 2 and 3 respectively. Finally, in this work are also incorporated 2 galaxies, (LEDA3085054 and [D97]ce-143), which belong to the C09 sample and for which spectroscopic data are available by IP03; all these spectra giving a final coverage of the C09 sample of $72 \%$.

\subsection{Abundance derivation}

The radiation emitted by a nebula depends on the abundances of the chemical elements and the physical state of the gas, specially its average temperature and density. So, physical and chemical properties of nebulae can be derived by measuring the intensities of collisionally excited emission lines. After hydrogen and helium, the most abundant element in the universe is oxygen. The oxygen abundance can be derived from because it exhibits emission lines in $\mathrm{H}$ II region spectra which are bright and easy to measure. In practice, the metallicity of SF galaxies is quantified via the oxygen abundance, as $12+\log (\mathrm{O} / \mathrm{H})$.

Prior to the determination of the gas-phase chemical abundances, the excitation conditions of the gas have to be explored. In order to do that we use the BPT diagrams (Baldwin et al. 1981). The derivation of gas-phase metal abundances is constrained to nebular emission-line spectra generated by photoionization from massive stars. Non-stellar ionizing sources, such as active galactic nuclei (AGN), produce generally distinctive emission-line ratios compared to ordinary $\mathrm{H}$ II regions. In Fig. 6] we present the BPT diagram with Kauffmann et al. (2003) empirical demarcation curve with continuous blue line, and Kewley et al. (2001) theoretical upper limit for starburst galaxies with dashed black line, in order to identify non-stellar ionizing sources. We use four distinctive colors/symbols for our sample of galaxies. With blue filled circles we plot the 16 integrated dwarf/irregular galaxies $\left(\mathrm{M}_{\mathrm{B}}>-19\right)$ of

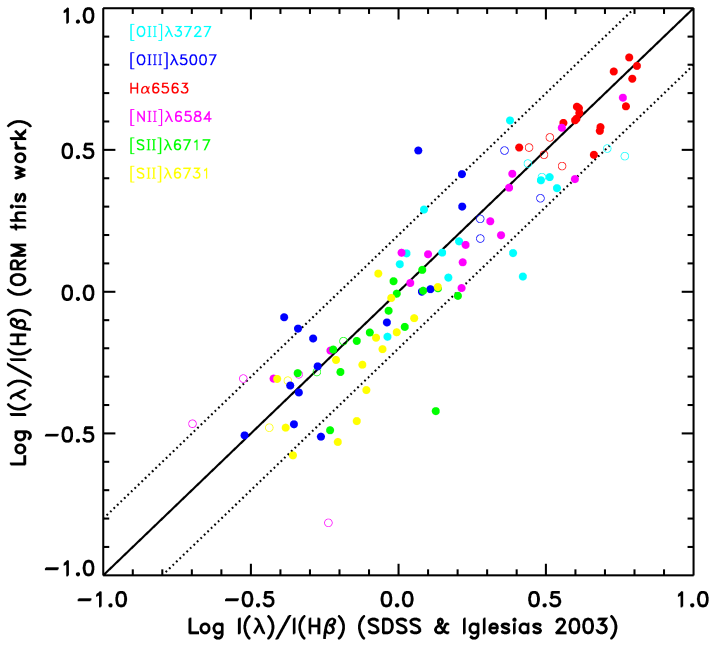

Fig. 5.- Emission line ratios $\mathrm{I}(\lambda) / \mathrm{I}(\mathrm{H} \beta)$ for the principal emission lines (without applying reddening correction) as derived by the ORM long-slit spectra observed for this work versus corresponding ratios from SDSS (filled points) or IP03 spectra (open cycles) for the same galaxy regions. Cyan: [O II] $\lambda 3727$, red: $\mathrm{H} \alpha$ 6563, blue: [O III] $\lambda 5007$, pink: [N II] $\lambda 6583$, green: [S II] $\lambda 6717$ and yellow: [S II] $\lambda 6731)$. The continuous line is the line of equality and the dotted lines indicate a variation by \pm 0.2 dex. 
our sample6. With red stars we plot the nuclei of 6 spirals and with green triangles their corresponding disk regions. Magenta squares are used for luminous $\left(\mathrm{M}_{\mathrm{B}} \leq-19\right)$ galaxies for which we integrate the $2 \mathrm{D}$ long-slit spectrum into one $1 \mathrm{D}$ spectrum (PGC057077 is ploted with magenta squares and PGC057064 with blue filled circles, although the spectra of these peculiar galaxies have been divided into two parts each, for different reasons, see Appendix for details). Although we have divided the spectrum of the irregular galaxy LEDA084703 into three parts, from now on we consider the integrated values, because it shows homogeneous chemical composition (see Appendix for details on this galaxy). In Fig. 6 we see that the nuclei of NGC6045, NGC6050A, KUG1602+175A, galaxy CGCG108+149 and the southern-east part of the peculiar object PGC057064 (see Appendix for special notes on this object) belong to the transition zone between the two separation curves. IC1182 lies on the separation curve of Kauffmann et al. (2003) and there is a controversy in the literature whether there is an AGN hosted in the nucleus of this merger (see Radovich et al. (2005) and references therein), but our long-slit spectrum is slightly off-set from the optical center. A detailed investigation of the nature of this peculiar object is out of the scope of the present paper and it will be presented in a forthcoming work (Petropoulou et al, in prep). The nucleus of IC1173 is clearly lying in the AGN region and we do not include it in our abundance analysis. We mark with a square the galaxy SDSS J160531.84+174826.1. It has been claimed (Dong et al. 2007) that this galaxy hosts a Seyfert 1 AGN; the position of this galaxy in the BPT diagram, set by the ratios of the lines in our emission line spectrum, is consistent with the area of the plot populated by SF objects.

In Fig. 7 we show the histograms of the distribution of the N2S27 and R238 parameters for our sample of galaxies, using the same colors

\footnotetext{
${ }^{6}$ In figures where the ploted quantities involve measurement of [N II] and [S II] lines, the galaxy LEDA3085054 is not included

${ }^{7} \mathrm{~N} 2 \mathrm{~S} 2=\log \left(\mathrm{I}_{[\mathrm{NII}] \lambda 6584} / \mathrm{I}_{[\mathrm{SII}] \lambda \lambda 6717.6731}\right)$ (see Pérez-Montero \& Contini 2009)

${ }^{8} \mathrm{R} 23=\mathrm{R} 2+\mathrm{R} 3$,

where $\mathrm{R} 2=\mathrm{I}_{[\mathrm{OII}] \lambda 3727+\lambda 3729} / \mathrm{I}_{\mathrm{H} \beta}$

and $\left.\mathrm{R} 3=\mathrm{I}_{[\mathrm{OIII}]}\right] 4959+\lambda 5007 / \mathrm{I}_{\mathrm{H} \beta}$.

When [O III] $\lambda 4959$ is not measured, we assume the theoretical ratio: $[\mathrm{O} \mathrm{III}] \lambda 4959 /[\mathrm{O} \mathrm{III}] \lambda 5007=0.33$
}

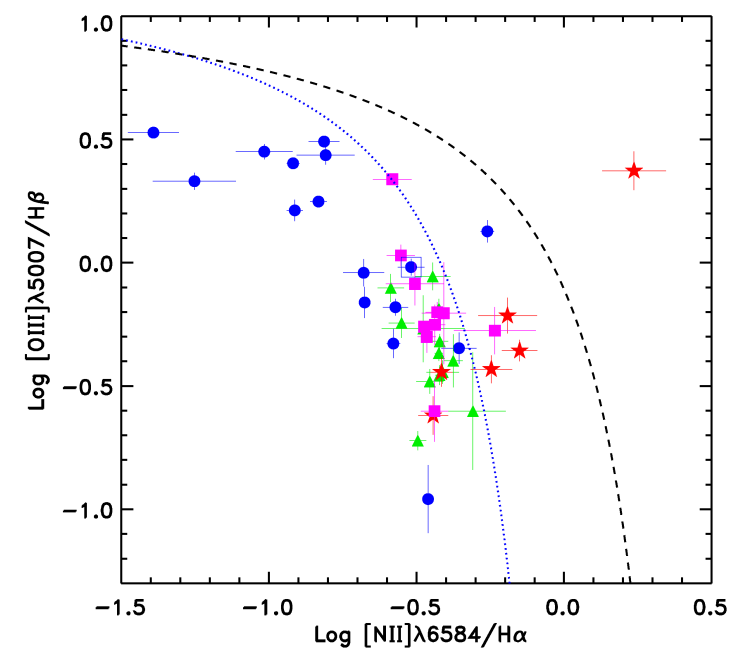

Fig. 6.- BPT diagram and Kauffmann et al. (2003) (continuous blue) and Kewlev et al. (2001) (dashed black) separation curves between objects with ionized gas produced by photoionization from massive stars and from non-stellar ionizing sources. We use four distinctive colors/symbols for our galaxies: blue filled circles for dwarf/irregular galaxies $\left(M_{B}>-19\right)$, magenta squares for luminous $\left(\mathrm{M}_{\mathrm{B}} \leq-19\right)$ but integrated galaxies, red stars for the nuclei of 6 spirals that we divide into different parts and green triangles for their corresponding disk components. The two parts of PGC057077 are plotted in magenta filled squares; similarly, the two parts of PGC057064 are plotted in blue filled circles. We mark with a square the galaxy SDSS J160531.84+174826.1 for which which it has been claimed (Dong et al. 2007) to host a Seyfert 1 AGN. 

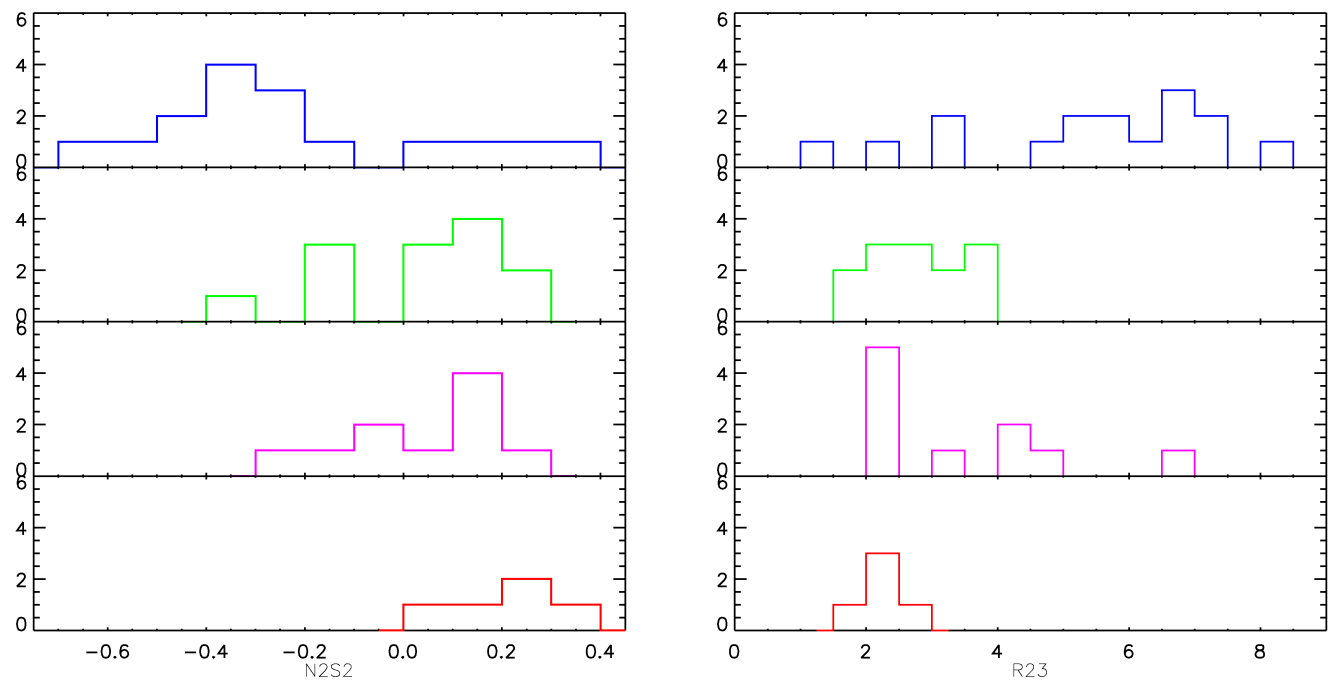

Fig. 7.- The histograms of the distribution of the N2S2 (left) and R23 (right) parameters for our sample of galaxies, using the same colors to separate out our sample in four categories as in Fig. 6. The histograms in both left and right panel correspond to top to bottom: dwarf/irregulars, disks of spirals, integrated spirals, nuclei of spirals.

to separate out our sample in four categories as in Fig. 6. The histograms in both left and right panel starting from the top correspond to: dwarf/irregulars, disks of spirals, integrated spirals, nuclei of spirals. The most populated N2S2 bin for the dwarf/irregular galaxies of our sample (left panel, first histogram from the top, with blue line) lies around $\mathrm{N} 2 \mathrm{~S} 2=-0.4$, although there are few objects sorting out to higher values. These high values, as we will see later in the discusion, seem consistent with the properties of these galaxies. Spiral galaxies are separated into 3 categories. The galaxies for which we only have integrated spectrum (magenta line, third from the top) show N2S2 values extending up to 0.4 and the most populated range is around 0.1-0.2. The other two categories correspond to massive galaxies divided into nuclei (red line, forth from the top) and disks (green, second from the top). Although both distributions show a common range of $\mathrm{N} 2 \mathrm{~S} 2$ values from 0.0 to 0.3 , there is a tendency for the spectra of the central parts to present higher N2S2 than the disks which can reach values as low as -0.3. In the same line for the R23 distribution, the dwarfs (right panel, first histogram from the top, with blue line) present the larger values of R23 up to 8 with few objects presenting values as low as R23 = 1-3 (as we will show later this is a hint on the nature of these objects). However, as a possible consequence of the bi-valuated nature of the $\mathrm{O} / \mathrm{H}$ vs $\mathrm{R} 23$ function, the separation among the bright galaxies is not as clear as in the left panel. Nonetheless it can be seen that, for integrated bright galaxies (magenta, third from the top), R23 distribution shows a large range with values from 2 to 7 with the most populated values close to 2; for the spatially resolved galaxies, the range of disks reaches higher values (up to 4) compared to nuclei (up to 3). This analysis highlights the fact that using SDSS spectroscopy, which most probably means sampling only galaxy centers (bottom histogram, with red line), could introduce a bias in the abundance results.

There are two commonly used methods to determine oxygen abundances in H II regions. The direct method is founded on a direct measurement of the electron temperature, applicable when the collisionally excited lines such as [O III $] \lambda 4363$, $[\mathrm{NII}] \lambda 5755,[\mathrm{SIII}] \lambda 6312$ are measured. We do not detect any of the temperature diagnostic lines in our spectra. In addition, considering Hercules distance and the resolution of our observations, we are constrained to integrate galaxy spectra over large spatial scales, in some cases containing sev- 
eral HII regions, with potentially different ionization conditions. In these cases, the use of the direct method to derive abundances could result misleading (e.g Kobulnicky et al. 1999).

The other way to determine oxygen abundances in HII regions is to use semi-empirical calibrations. There are two different types of calibrations: the empirical ones, based on fits to objects for which an accurate direct derivation of $\mathrm{O} / \mathrm{H}$ is available (e.g. Pettini \& Pagel 2004; Nagao et al. 2006; Pérez-Montero \& Contini 2009; Pilyugin \& Thuan 2005; Pilyugin et al. 2010) and the ones based on predictions of theoretical photoionization models (e.g. McGaugh 1991; Kewley \& Dopita 2002; Tremonti et al. 2004). For an extensive review see Lopez-Sanchez \& Esteban (2010) and Kewley \& Ellison (2008).

It has been shown recently (Bresolin et al. 2009) that model calibrations show a positive shift of 0.3 dex compared to oxygen abundances derived with the direct method, whereas the latter agree very well with oxygen abundances derived for the massive young stars. For our sample we also find a 0.3 dex positive shift between the abundances predicted using McGaugh (1991) model calibration (formulated by Kobulnicky et al. 1999) and the empirical calibration calculated by Pilyugin et al. (2010). Taking this into account, in this analysis we have chosen using empirical calibrations.

Our sample spans from very low metallicity dwarfs to evolved disks and galaxy centers, with an important fraction lying in the turnover region of the R23 versus oxygen abundance relation (see Fig. 7). Among the empirical calibrations we use the N2-calibration given by Pérez-Montero \& Contini (2009) (from now on PMC09) and the recent calibration by Pilyugin et al. (2010) (from now on P10), because they have the advantage of being valid along the whole metallicity range of our sample. The N29 PMC09 parameter shows a monotonic relation with oxygen abundance, thus avoiding the degeneracy problem of R23; though this calibration involves a large rms error of up to $\sim 0.3$ dex. In order to circumvent this problem P10 has provided with a new improved calibration which uses a multiparametric function of $\mathrm{P}, \mathrm{R} 2, \mathrm{R} 3, \mathrm{~S} 2, \mathrm{~N} 210$

\footnotetext{
${ }^{9}$ Defined by PMC09 as N2 $=\log \left(\mathrm{I}_{[\mathrm{NII}] \lambda 6584} / \mathrm{I}_{\mathrm{H} \alpha}\right)$

${ }^{10}$ Defined in $\mathrm{P} 10$ as $\mathrm{N} 2=\mathrm{I}_{[\mathrm{NII}] \lambda 6548+\lambda 6584} / \mathrm{I}_{\mathrm{H} \beta}$,
}

parameters and produces a very small rms error $\sim 0.07$ dex. In Table 4 we give the oxygen abundance derived using both abundance calibrations. We are aware that using the nitrogen as an abundance indicator to derive oxygen abundances can be potentially dangerous in case of objects with particular evolution, for example for nitrogen enriched galaxies (see PMC09). Comparing both abundance calibrations, we interpret PMC09 abundances as an upper estimate while P10 as a lower estimate and, being conservative, we have adopted the mean of the two oxygen abundances. For each galaxy of our sample the adopted $\mathrm{O} / \mathrm{H}$ value is consistent with the prediction of both calibrations within the statistical errors. For each $\mathrm{O} / \mathrm{H}$ determination we have estimated the corresponding error as the rms of a gaussian error distribution produced by a random sampling simulation taking into account the errors of the $\mathrm{P}$, R23, R2, R3, S2, and N2 parameters. This error estimation has been adopted in all cases except in those cases for which it is less than the statistical error provided by the P10 calibration $(\sim 0.07$ dex), which was finally adopted. As can be noted in Table 4, the error adopted is very close to the half of the difference in $\mathrm{O} / \mathrm{H}$ provided by both calibrations.

Nitrogen is also an important element to investigate galaxy evolution. Nitrogen abundance deserves a more thorough determination; in particular the importance of possible self-enrichment Lopez-Sanchez \& Esteban 2010; Monreal-Ibero et al. 2010) and the differential chemical evolution of $\mathrm{N}$ versus $\mathrm{O}$ could add some degree of uncertainty. These effects have been extensively considered by PMC09, thus in this work we have adopted their calibration for nitrogen given by the N2S2 parameter which has a typical rms error of $\sim 0.3$ dex. In Table 4 the $\mathrm{N} / \mathrm{O}$ values are also presented.

For the spatially resolved spirals of our sample we have explored the abundance gradients. Figs. 8, 9 show the $\mathrm{O} / \mathrm{H}$ and $\mathrm{N} / \mathrm{O}$ abundance profiles, respectively, for the 6 spiral galaxies of our sample for which individual spectra, corresponding to different spatial regions, have been ex-

$\mathrm{S} 2=\mathrm{I}_{[\mathrm{SII}] \lambda 6717+\lambda 6731} / \mathrm{I}_{\mathrm{H} \beta}$, and $\mathrm{P}=\mathrm{R} 3 / \mathrm{R} 23$.

The theoretical ratio [N II] $\lambda 6548 /[\mathrm{N} \mathrm{II}] \lambda 6584=0.3$ is assumed when necessary. 
TABLE 4

Chemical Abundances

\begin{tabular}{|c|c|c|c|c|c|}
\hline GALAXY & $\begin{array}{c}12+\log (\mathrm{O} / \mathrm{H}) \\
\text { PMC09 }\end{array}$ & $\begin{array}{c}12+\log (\mathrm{O} / \mathrm{H}) \\
\mathrm{P} 10\end{array}$ & $\begin{array}{c}12+\log (\mathrm{O} / \mathrm{H}) \\
\text { adopted }\end{array}$ & $\begin{array}{c}\text { error } \\
\text { adopted }\end{array}$ & $\begin{array}{c}\log (\mathrm{N} / \mathrm{O}) \\
\mathrm{PMC09}\end{array}$ \\
\hline PGC057185a & 8.64 & 8.48 & 8.56 & 0.07 & -1.04 \\
\hline PGC057185b & 8.74 & 8.53 & 8.63 & 0.07 & -0.72 \\
\hline PGC057185c & 8.69 & 8.50 & 8.60 & 0.12 & -0.86 \\
\hline IC1173a & 8.83 & 8.56 & 8.69 & 0.26 & -0.63 \\
\hline IC1173e & 8.72 & 8.57 & 8.65 & 0.07 & -1.05 \\
\hline KUG1603+179Aa & 8.73 & 8.53 & 8.63 & 0.07 & -0.74 \\
\hline KUG1603+179Ab & 8.88 & 8.57 & 8.72 & 0.07 & -0.54 \\
\hline KUG1603+179Ac & 8.74 & 8.53 & 8.64 & 0.07 & -0.69 \\
\hline NGC6050Aa & 8.92 & 8.53 & 8.72 & 0.07 & -0.55 \\
\hline NGC6050Ab & 8.71 & 8.59 & 8.65 & 0.07 & -0.69 \\
\hline NGC6050B & 8.63 & 8.52 & 8.58 & 0.07 & -1.02 \\
\hline LEDA1543586 & 8.43 & 8.34 & 8.38 & 0.07 & -1.15 \\
\hline NGC60545a & 8.77 & 8.49 & 8.63 & 0.09 & -0.71 \\
\hline NGC6045b & 8.74 & 8.54 & 8.64 & 0.07 & -0.57 \\
\hline NGC6045c & 8.95 & 8.54 & 8.75 & 0.07 & -0.36 \\
\hline NGC6045d & 8.68 & 8.61 & 8.65 & 0.07 & -0.53 \\
\hline NGC6045e & 8.74 & 8.46 & 8.60 & 0.07 & -0.74 \\
\hline KUG1602+174A & 8.72 & 8.58 & 8.65 & 0.12 & -0.81 \\
\hline LEDA084719 & 8.75 & 8.46 & 8.61 & 0.26 & -0.69 \\
\hline PGC057077a & 8.73 & 8.46 & 8.60 & 0.07 & -0.64 \\
\hline PGC057077b & 8.70 & 8.54 & 8.62 & 0.07 & -0.63 \\
\hline UGC10190a & 8.75 & 8.53 & 8.64 & 0.07 & -1.01 \\
\hline UGC10190b & 8.72 & 8.58 & 8.65 & 0.07 & -0.86 \\
\hline UGC10190c & 8.61 & 8.50 & 8.55 & 0.07 & -1.27 \\
\hline LEDA140568 & 8.08 & 8.05 & 8.07 & 0.11 & -1.60 \\
\hline [D97]ce-200 & 8.35 & 8.19 & 8.27 & 0.07 & -1.49 \\
\hline PGC57064a & 8.79 & 8.50 & 8.64 & 0.07 & -0.58 \\
\hline PGC057064b & 8.86 & 8.35 & 8.61 & 0.07 & -0.81 \\
\hline LEDA084703int & 8.41 & 8.28 & 8.35 & 0.07 & -1.29 \\
\hline LEDA084703a & 8.40 & 8.24 & 8.32 & 0.07 & -1.29 \\
\hline LEDA084703b & 8.42 & 8.29 & 8.35 & 0.07 & -1.25 \\
\hline LEDA084703c & 8.46 & 8.26 & 8.36 & 0.07 & -1.40 \\
\hline KUG1602+175 & 8.72 & 8.54 & 8.63 & 0.07 & -0.63 \\
\hline LEDA084710 & 8.67 & 8.44 & 8.56 & 0.27 & -0.92 \\
\hline CGCG108-149 & 8.88 & 8.53 & 8.71 & 0.12 & -0.61 \\
\hline KUG1602+174B & 8.70 & 8.54 & 8.62 & 0.07 & -0.87 \\
\hline LEDA084724 & 8.53 & 8.42 & 8.48 & 0.07 & -1.24 \\
\hline SDSSJ160556.98+174304.1 & 8.27 & 8.21 & 8.24 & 0.11 & -1.36 \\
\hline$[\mathrm{DKP} 87] 160310.21+175956.7$ & 8.61 & 8.50 & 8.56 & 0.07 & -1.19 \\
\hline SDSSJ160524.27+175329.3 & 7.97 & 8.01 & 7.99 & 0.07 & -1.67 \\
\hline SDSSJ160523.66+174832.3 & 8.54 & 8.28 & 8.41 & 0.07 & -1.30 \\
\hline IC1182 & 8.61 & 8.41 & 8.51 & 0.07 & -1.19 \\
\hline IC1182:[S72]d & 8.34 & 8.27 & 8.31 & 0.07 & -1.29 \\
\hline SDSSJ160531.84+174826.1 & 8.66 & 8.64 & 8.65 & 0.07 & -0.45 \\
\hline SDSSJ160304.20+171126.7 & 8.62 & 8.44 & 8.53 & 0.07 & -1.10 \\
\hline SDSSJ160520.58+175210.6 & 8.71 & 8.68 & 8.69 & 0.15 & -0.64 \\
\hline [D97]ce-143 & 8.43 & 8.26 & 8.35 & 0.07 & -1.40 \\
\hline LEDA3085054 & $\ldots$ & $7.43^{\mathrm{a}}$ & 7.58 & 0.07 & $-0.86^{\mathrm{a}}$ \\
\hline
\end{tabular}

Note.- The oxygen abundance derived using Pérez-Montero \& Contini (2009) and Pilvugin et al. (2010) calibrations. N/O is derived using N2S2 calibration by Pérez-Montero \& Contini (2009).

a These values are given by the used calibration assuming upper limits for the [N II] and [S II] lines. The adopted $\mathrm{O} / \mathrm{H}$ value is the one provided by Iglesias-Páramo et al. (2003) calculated using the P-method; we see that is in very good agreement with the $O / H$ value that we calculate with the P10 method. 

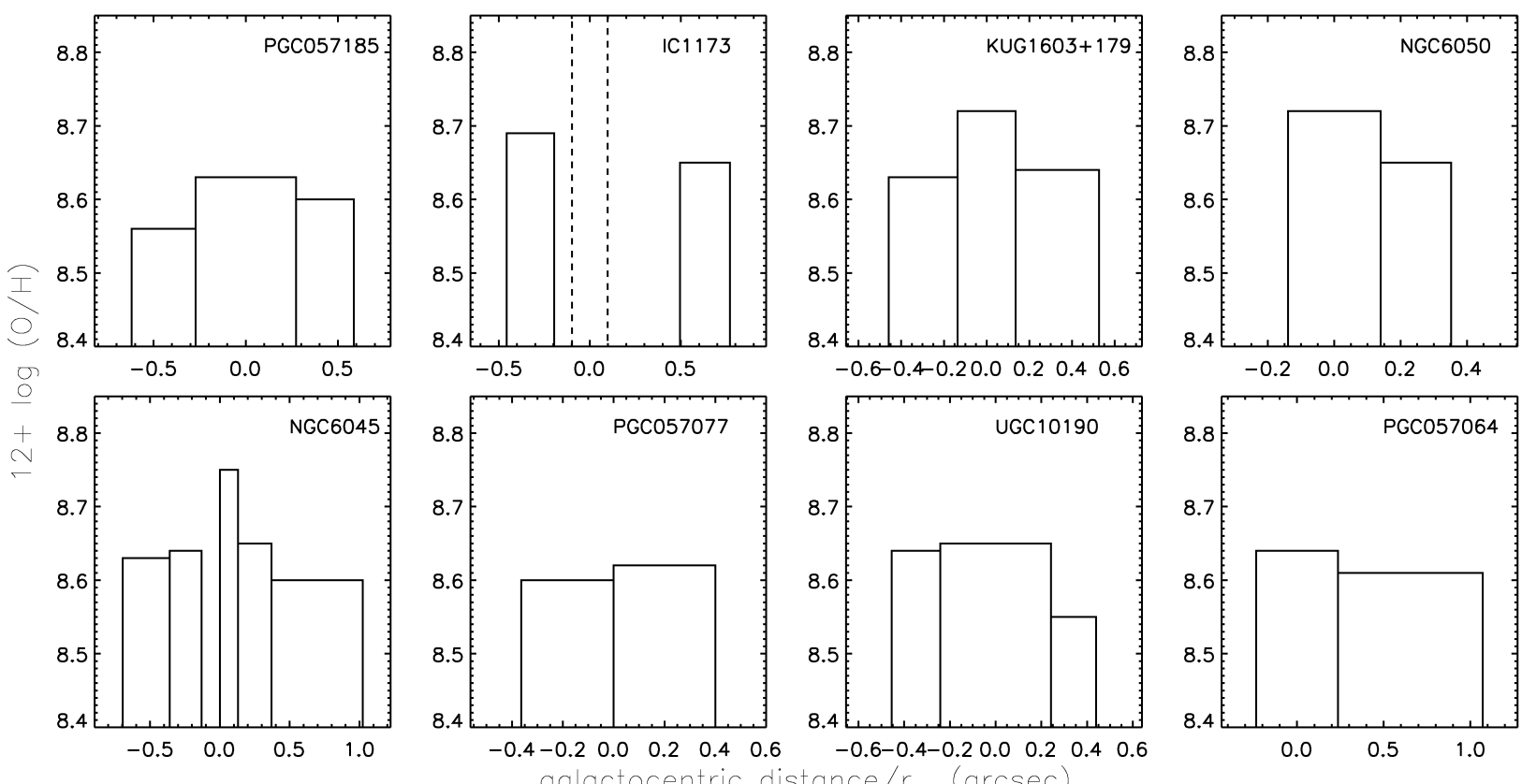

Fig. 8.- Oxygen abundances of the galaxies with spatially resolved spectroscopy in radial bins of galactocentric distance in arcsec normalized to $r_{25}$. For IC1173, dashed lines indicate the radial bin of the AGN extracted spectrum.

tracted. We plot the chemical abundances derived for each region in bins of galactocentric distance in arcsec, normalized to the $r_{25}$ radius 11 extracted from SDSS r' band images (see Table 5). The plots show mild or flat $\mathrm{O} / \mathrm{H}$ abundance gradients across a limited radial extension of the disk

\subsection{Physical Properties}

We derive stellar mass for each galaxy using the kcorrect_v4 algorithm (version 4_2) of Blanton \& Roweis (2007). This IDL code corrects implicitly for dust and assumes a universal IMF of Chabrier (2003) form. The SEDs used to best fit the observed SDSS ugriz photometry are based on Bruzual \& Charlot (2003) stellar evolution synthesis codes. The best-fitting SED provides an estimate of the stellar mass-to-light ratid 12 . Drorv et al. (2004) argue that broadband color mass estimators, can yield fairly reliable stellar

${ }^{11}$ Radius $r_{25}$ is the radius of the galaxies at a surface brightness of $25 \mathrm{mag} / \operatorname{arcsec}^{2}$.

${ }^{12}$ The $\chi^{2}$ of the fit is less than 5 (1) for $73 \%(27 \%)$ of our sample masses for galaxies within $\sim 0.2$ dex over almost 4 decades in mass. Li \& White (2009) demonstrate that, once all estimates are adapted to assume the same IMF, the Blanton \& Roweis (2007) masses agree quite well with those obtained from the single-colour estimator of Bell et al. (2003) and also with those derived by Kauffmann et al. (2003) from a combination of SDSS photometry and spectroscopy. More recently, Jabran Zahid et al. (2010) also state that optical bands are sufficient to constrain the SED fit for determining masses of $\mathrm{SF}$ galaxies. The galaxy stellar masses given from the algorithm were converted to $\mathrm{H}_{0}=73 \mathrm{~km} \mathrm{~s}^{-1}$ $\mathrm{Mpc}^{-1}$ adopted in this work and are presented in Table 5 .

We also calculate the two-dimensional local galaxy density $\Sigma$, using two methods. We use the density estimator based on an average of the projected distances (d, in Mpc) to the fourth and fifth nearest neighbor as given by Mouhcine et al. (2007) and used by Ellison et al. (2009)

$$
\log \Sigma_{4,5}=\frac{1}{2} \log \left(\frac{4}{\pi d_{4}^{2}}\right)+\frac{1}{2} \log \left(\frac{5}{\pi d_{5}^{2}}\right)
$$



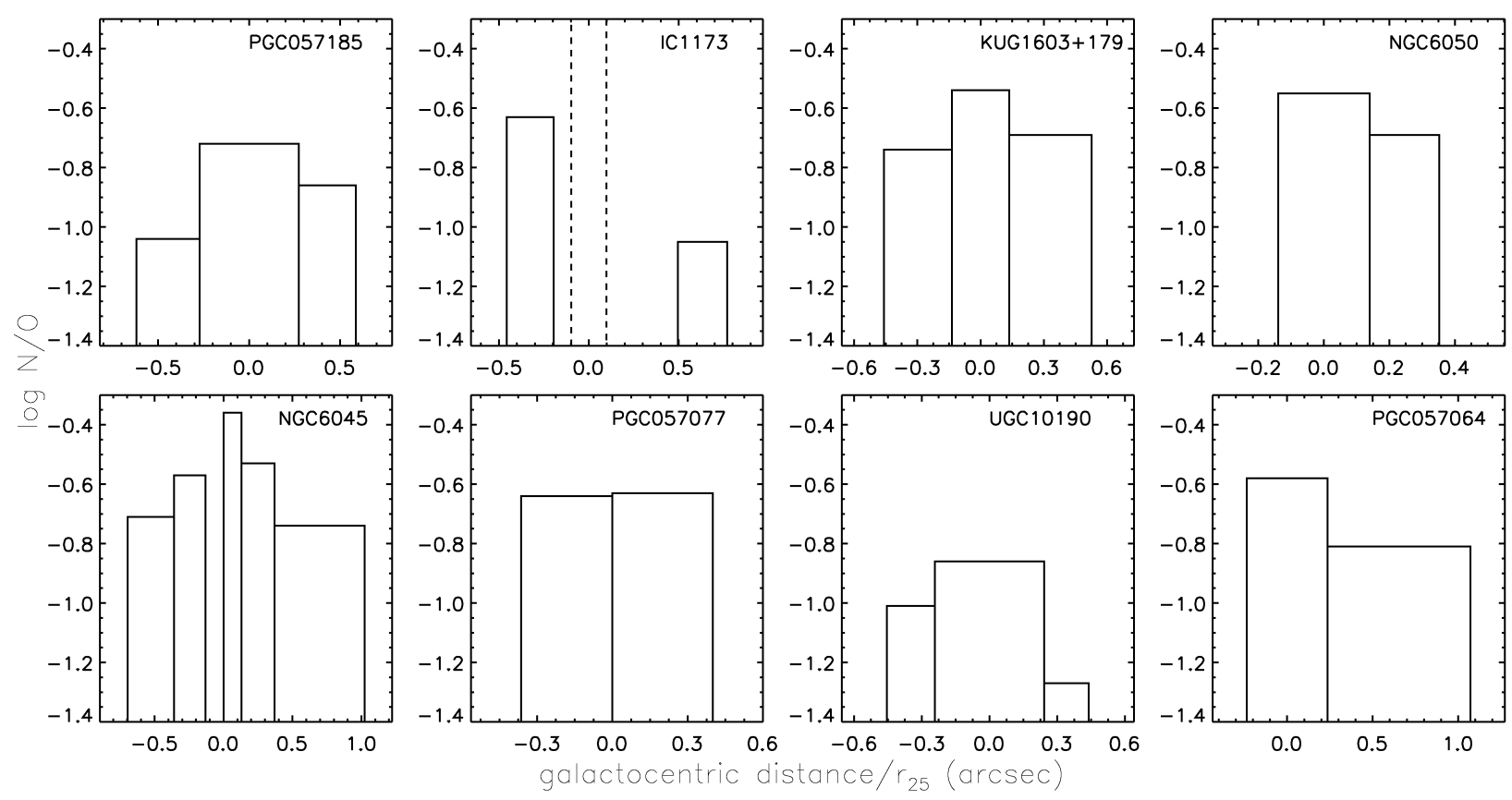

Fig. 9. - The same as in Fig. 8 for N/O.

considering all galaxies with SDSS spectra in the Hercules region at the corresponding redshift range, and presenting a difference in radial velocity smaller than $750 \mathrm{~km} / \mathrm{s}$, a value approximately equal to $\sigma_{\mathrm{V}}$ of the cluster.

To account for possible fiber collisions which may lead to an under-estimate of the local density in regions rich in projected galaxies as cluster centers, we calculate the local galaxy number density to the $10^{\text {th }}$ nearest neighbor $\Sigma_{10}$, using a magnitude limited sample of galaxies taken out from SDSS database without velocity constrain. Counts of galaxies as a proxy of local environment has the main weakness that is missing the luminosity information of the counted galaxies. To account for this problem, we limit to luminous galaxies $M_{r^{\prime}}=-19, m_{r^{\prime}}=17 \mathrm{mag}$ when corrected for galactic extinction. To correct for background and foreground galaxies, we use the field galaxy counts as given by Yasuda et al. (2001). The local galaxy number density is calculated to the $10^{\text {th }}$ neighbor in order to improve statistics. As expected, the corresponding densities were higher, but they do not affect the conclusions of the MZ relation analysis as will be discused in 95 . The density estimates $\log \Sigma_{4,5}$ and $\log \Sigma_{10}$ are quoted in Table 5.

We then estimate the projected distance of our galaxies to the cluster center. As already mentioned in $\$ 1$. Hercules is a peculiar cluster of galaxies, where the maximum of the cluster galaxy distribution $\left(16^{\mathrm{h}} 05^{\mathrm{m}} 15.0^{\mathrm{s}}+17^{\mathrm{d}} 44^{\mathrm{m}} 55^{\mathrm{s}}\right.$, extracted from NED) is not coincident with the primary Xray maximum found by Huang \& Sarazin (1996). The center of the brightest X-ray component coincides with the brightest cluster galaxy NGC6041A $\left(16^{\mathrm{h}} 04^{\mathrm{m}} 35.8^{\mathrm{s}}+17^{\mathrm{d}} 43^{\mathrm{m}} 18^{\mathrm{s}}\right)$. In Table 5 we give the projected distance of our sample galaxies to both centers in Mpc, referred to the cosmological corrected distance of Hercules cluster 158.3 Mpc.

We calculate the SFR of our galaxies, from their $\mathrm{H} \alpha$ emission given by $\mathrm{C} 09$ using the Kennicutt (1998) calibration. We use $\mathrm{c}(\mathrm{H} \beta)$ derived by our optical spectroscopy to correct $\mathrm{H} \alpha$ emission from extinction, assuming the Miller \& Mathews (1972) extinction law with $R_{V}=3.2$. When spectra of different parts of a galaxy are considered, the $\mathrm{c}(\mathrm{H} \beta)$ used to calculate the global SFR of the galaxy is the mean value of all these $\mathrm{c}(\mathrm{H} \beta)$ derived spectroscopically. We also correct the $\mathrm{H} \alpha$ flux from [N II] contamination using the empirical 
correction given by Reverte (2008):

$$
\begin{aligned}
\log \mathrm{EW}(\mathrm{H} \alpha) & =(-0.34 \pm 0.03)+ \\
(1.13 & \pm 0.02) \log \mathrm{EW}\left(\mathrm{H} \alpha+\left[\mathrm{N}_{\mathrm{II}}\right]\right)
\end{aligned}
$$

This empirical correction was derived using line fluxes integrated for entire galaxies, extracted from the extended spectrophotometric galaxy sample of Jansen et al. (2000), suitable for this kind of analysis. The errors on SFR quoted take into account only the $\mathrm{H} \alpha$ flux error.

Finally we note that the HI survey of Dickey (1997) covers the whole central region of A2151 where all our SF galaxies lie. We have adopted the HI masses provided in that work (converting them to $\mathrm{H}_{0}=73 \mathrm{~km} \mathrm{~s}^{-1} \mathrm{Mpc}^{-1}$ ).

Table 5 summarizes for each galaxy the physical properties calculated in this work and others extracted from the literature: the $\mathrm{M}_{\mathrm{B}}$ absolute magnitude, the galaxy radius at $25 \mathrm{mag} \operatorname{arcsec}^{-2}$ in arcsec (extracted from the SDSS $i$ so $_{A}$ parameter for the r' band), the local density estimates $\log \Sigma_{4,5}$ and $\log \Sigma_{10}$, the projected distance to the cluster center and the projected distance to the $\mathrm{X}$-Rays center in Mpc, the stellar mass calculated with kcorrect algorithm, the HI mass from Dickey (1997), and the SFR in $M_{\odot} \mathrm{yr}^{-1}$.

\section{DISCUSSION}

\subsection{Metallicity vs Local Density}

In this paper we study the relation between metallicity and environment for our sample of SF galaxies in the Hercules cluster. We examine the potential impact of the environment on the $\mathrm{MZ}$ and LZ relations and investigate different evolutionary scenarios of these cluster galaxies.

The Hercules cluster three dimensional galaxy distribution is extremely clumpy (Bird et al. 1995), rendering it a very interesting vivid environment difficult to be disentangled only in the projected space without taking into account galaxy velocity. The local density of galaxies has been widely used as an indicator of their environment; in this work we have studied the behavior of metallicity versus local density. The local density $\Sigma_{4,5}$, derived to the $4^{t h}$ and $5^{t h}$ nearest neighbor would describe the local environment at the group-scale.
In Fig. 10 we plot the galaxy gas-phase oxygen abundances versus the local density estimators $\Sigma_{4,5}$ (left) and $\Sigma_{10}$ (right) calculated in 4.4 We keep the same color/symbol distinction as in Fig. 6. Additionally, the points corresponding to the same galaxy (nuclei and disks), are connected with straight lines. We see that for luminous galaxies, the oxygen abundance does not show any significant dependence on the local galaxy number density. Luminous galaxies of this sample span the whole range of densities and all are found to have nearly solar oxygen abundance. Dwarf galaxies show a noticeable variation: the $\sim 80 \%$ of the less metallic dwarfs $(12+\log \mathrm{O} / \mathrm{H}<8.4)$ are located at $\Sigma_{4,5}<1.85$, whereas the $\sim 70 \%$ of the higher metallicity $(12+\log \mathrm{O} / \mathrm{H}>8.4)$ dwarf galaxies are located at very high local densities $\Sigma_{4,5}>1.85$. A substantial fraction of these more metallic dwarfs have been identified to be affected by interactions and they are described in detail in the Appendix. This dual behavior is not so evident when $\Sigma_{10}$ density estimator is used. The dependency observed in Fig. 10 of the metallicity of dwarf/irregular galaxies with local density could be interpreted as follows: at the highest local densities, i.e. approaching the cluster center, only the more "robust" -i.e. more massive and more metallic- dwarf galaxies can survive. Conversely, the less metallic dwarf galaxies should have been incorporated recently to the cluster. This "newcomers" scenario for dwarfs is additionally supported by the fact that the majority of the low metallicity dwarf galaxies present radial velocities which differ from the radial velocity of the brighter cluster galaxy NGC6041A -located at the X-ray maximum of the cluster- by more than $\sigma_{\mathrm{V}}$, possible evidence of infall (\$2.1).

One point worth of mentioning here is that, in this work, we study the effect of local galaxy density sampling much denser environments (from $\log \Sigma_{4.5}=1.0$ to 2.5$)$ than in previous works (Mateus et al. 2007; Mouhcine et al. 2007; Cooper et al. 2008; Ellison et al. 2009) typically reaching $\log \Sigma \sim$ 1.5. Additionally, the works mentioned above use the SDSS database and include few dwarf galaxies, mainly due to the magnitude limit of SDSS in combination with galaxy-size limits or redshift constraints applied in order to minimize possible aperture effects. This study, therefore, is complementary and goes beyond these previous works, 
TABLE 5

Physical PRoperties

\begin{tabular}{|c|c|c|c|c|c|c|c|c|c|}
\hline Galaxy & $\begin{array}{l}\mathrm{M}_{\mathrm{B}} \\
\mathrm{mag}\end{array}$ & $\begin{array}{c}i s O_{A} \\
\operatorname{arcsec}\end{array}$ & $\log \Sigma_{4,5}$ & $\log \Sigma_{10}$ & $\begin{array}{c}R \\
\mathrm{Mpc}\end{array}$ & $\begin{array}{c}R_{X} \\
\mathrm{Mpc}\end{array}$ & $\begin{array}{c}M_{\star} \\
10^{8} M_{\odot}\end{array}$ & $\begin{array}{c}M_{H I} \\
10^{8} M_{\odot}\end{array}$ & $\begin{array}{c}\mathrm{SFR} \\
M_{\odot} y r^{-1}\end{array}$ \\
\hline PGC057185 & -19.51 & 24.24 & 1.4 & 1.5 & 1.06 & 1.47 & 169.8 & $\ldots$ & $0.8 \pm 0.1$ \\
\hline IC 1173 & -20.79 & 30.68 & 1.1 & 1.5 & 0.9 & 0.92 & 444.7 & 37.3 & $7.3 \pm 0.7$ \\
\hline KUG1603+179A & -20.05 & 23.54 & 2.0 & 2.4 & 0.18 & 0.62 & 275.9 & 128.5 & $3.9 \pm 0.2$ \\
\hline NGC6050A & -20.87 & 30.10 & 1.7 & 2.4 & 0.1 & 0.53 & 27.6 & 53.1 & $\ldots$ \\
\hline NGC6050B & -19.30 & $\ldots$ & 2.3 & 2.4 & 0.1 & 0.53 & 262.7 & $\ldots$ & $\ldots$ \\
\hline LEDA1543586 & -18.46 & 13.43 & 1.8 & 1.9 & 0.3 & 0.53 & 6.3 & 27.6 & $0.19 \pm 0.01$ \\
\hline NGC6045 & -21.04 & 36.76 & 2.0 & 2.4 & 0.08 & 0.37 & 806.9 & $46.8^{\dagger}$ & $15.2 \pm 1.0$ \\
\hline KUG1602+174A & -19.24 & 13.47 & 1.2 & 2.3 & 1.17 & 1.03 & 72.6 & $\cdots$ & $0.6 \pm 0.1$ \\
\hline LEDA084719 & -19.20 & 10.60 & 1.6 & 1.9 & 0.29 & 0.4 & 83.7 & $\cdots$ & $1.5 \pm 0.3$ \\
\hline PGC057077 & -19.11 & 11.00 & 2.0 & 2.2 & 0.22 & 0.65 & 53.3 & $\ldots$ & $8.8^{\ddagger}$ \\
\hline UGC10190 & -19.21 & 26.40 & 1.9 & 2.1 & 0.19 & 0.56 & 60.8 & 100.8 & $0.4 \pm 0.1$ \\
\hline LEDA140568 & -17.42 & 13.65 & 1.2 & 1.5 & 0.49 & 0.93 & 2.1 & 36.4 & $0.017 \pm 0.002$ \\
\hline$[D 97] \mathrm{ce}-200$ & -17.74 & 8.54 & 2.3 & 2.3 & 0.13 & 0.38 & 3.0 & 9.0 & $0.091 \pm 0.006$ \\
\hline PGC057064 & $-18.65^{\star}$ & $18.65^{\star}$ & 2.1 & 1.9 & 0.26 & 0.64 & $104.0^{\star}$ & $\ldots$ & $3.9 \pm 0.9^{\star}$ \\
\hline LEDA084703 & -18.79 & 15.94 & 1.6 & 1.7 & 2.13 & 1.81 & 37.9 & 26.5 & $0.73 \pm 0.03$ \\
\hline KUG $1602+175$ & -20.57 & 21.92 & 1.3 & 1.6 & 0.89 & 0.76 & 191.4 & 99.6 & $4.7 \pm 0.3$ \\
\hline LEDA084710 & -19.23 & 18.85 & 1.2 & 1.7 & 1.05 & 0.8 & 122.9 & $\ldots$ & $3.9 \pm 0.2$ \\
\hline CGCG108-149 & -20.14 & 18.87 & 1.3 & 1.6 & 0.97 & 1.39 & 311.5 & $15.5^{\dagger}$ & $3.2 \pm 0.3$ \\
\hline KUG1602+174B & -19.70 & 18.65 & 1.2 & 1.8 & 1.15 & 1.04 & 96.1 & 49.9 & $1.9 \pm 0.2$ \\
\hline LEDA084724 & -18.72 & 14.82 & 1.0 & 1.4 & 0.57 & 0.86 & 42.6 & $\ldots$ & $1.002 \pm 0.03$ \\
\hline SDSS J160556.98+174304.1 & -17.09 & 5.54 & 1.2 & 1.5 & 0.47 & 0.89 & 3.3 & $\cdots$ & $0.210 \pm 0.009$ \\
\hline$[\mathrm{DKP} 87] 160310.21+175956.7$ & -17.41 & 7.59 & 2.0 & 1.8 & 0.34 & 0.67 & 5.7 & $\cdots$ & $0.06 \pm 0.02$ \\
\hline SDSS J160524.27+175329.3 & -16.16 & 3.53 & 1.3 & 1.8 & 0.41 & 0.71 & 1.1 & $\cdots$ & $0.022 \pm 0.002$ \\
\hline SDSS J160523.66+174832.3 & -16.76 & 5.88 & 1.9 & 2.1 & 0.19 & 0.58 & 3.4 & $\cdots$ & $0.07 \pm 0.02$ \\
\hline IC1182 & -20.98 & 27.24 & 2.1 & 2.0 & 0.28 & 0.7 & 654.9 & $\ldots$ & $22.1 \pm 0.6$ \\
\hline IC1182:[S72]d & -17.73 & 9.62 & 1.9 & 1.9 & 0.33 & 0.76 & 1.3 & 186.0 & $0.83 \pm 0.02$ \\
\hline SDSS J150531.84+174826.1 & -17.23 & 5.21 & 2.2 & 2.0 & 0.25 & 0.66 & 15.5 & $\ldots$ & $0.146 \pm 0.009$ \\
\hline SDSS J160304.20+171126.7 & -17.42 & 9.05 & 1.6 & 1.7 & 2.1 & 1.78 & 24.8 & $\ldots$ & $0.55 \pm 0.03$ \\
\hline SDSS J160520.58+175210.6 & -18.51 & 13.85 & 1.9 & 1.9 & 0.34 & 0.64 & 10.6 & $\ldots$ & $0.20 \pm 0.02$ \\
\hline$[D 97]$ ce- 143 & -17.56 & 8.29 & 1.4 & 1.9 & 0.33 & 0.64 & 14.9 & 16.7 & $0.025 \pm 0.004$ \\
\hline LEDA3085054 & -16.23 & 4.65 & 1.4 & 1.5 & 0.46 & 0.88 & 0.7 & 19.7 & $0.045 \pm 0.002$ \\
\hline
\end{tabular}

Note.-Column1: galaxy name from C09; Column 2: $B$ absolute magnitude, calculated from SDSS g' magnitudes, assuming a distance to A2151 of $158.3 \mathrm{Mpc}$ and an average correction of $g^{\prime}-B=-0.3 \mathrm{mag}$ (Fukugita et al. 1995). All the quantities of this table are refered to the cosmological corrected distance of Hercules cluster assuming $\mathrm{H}_{0}=73 \mathrm{~km} \mathrm{~s}^{-1} \mathrm{Mpc}^{-1}$; Column 3 : $\mathrm{SDSS}_{\text {iso }}$ parameter for the r' band in arcsec; Column 4: local density estimator $\log \Sigma_{4,5}$; Column 5: local density estimator log $\Sigma_{10}$; Column 6: projected distance to the cluster center in Mpc; Column 7: projected distance to the X-Rays center in Mpc; Column 8: stellar mass calculated with kcorrect; Column 9: HI mass from Dickey (1997); Column 10: SFR in $M_{\odot} \mathrm{yr}^{-1}$.

${ }^{\dagger}$ For NGC6045 and CGCG108-149 Dickey (1997) data are incomplete or non existent, then we have used HI masses from Giovanelli et al. (1981). For NGC6045 HI measurements provided by these two works are consistent, for the galaxy part that was included in Dickey (1997) due to the velocity cut-off of the spectrometer used, see Appendix for details

${ }^{\ddagger}$ The derived SFR is uncertain, as this galaxy shows two parts suffering completely different extinctions. The average extinction has been used.

* These physical properties have been derived on the basis of integrated photometric data of this merger being considered as one galaxy. 
dealing also with the dwarf galaxy population. In this sense, the Hercules cluster, being an ideal laboratory to study the environmental effects on SF galaxies, was not included in the SDSS-DR4 used in all these previous studies.

\subsection{Mass and Luminosity vs Metallicity}

Fig. 11 (left) shows the gas-phase oxygen abundance versus galaxy stellar mass for our sample of galaxies (colors and point features as in Fig. 10). We see that Hercules SF galaxies follow a well defined sequence on this plot, which reaches a saturation value $\sim Z_{\odot}$ for galaxies with $\sim 10^{10} M_{\odot}$. We observe that the set of dwarfs/irregulars populating the higher local densities (i.e. $\log \Sigma_{4,5}>1.85$, see \$5.1), marked here with circles, appear shifted towards higher metallicities for their mass. This fact suggests a different evolution for these galaxies in the environment of the cluster, thus providing a physical reason for the dispersion in the MZ relation. These findings are in the line of the results of Cooper et al. (2008) who attribute $\sim 15 \%$ of the measured scatter of the MZ relation to the environment.

For the sake of clarity, in Fig. 11 (right) we added the 15 Virgo dIs and BCDs (light blue open circles) from Vaduvescu et al. (2007) and references therein (excluding VCC641 because of its uncertain oxygen abundance). For consistency with our data, when a direct abundance estimation is not available, we recalculate their abundances using the P10 method. We have recalculated the stellar mass for the Virgo dwarf galaxy sample using the kcorrect code (as for the Hercules sample) and we found a good agreement with the mass estimate given by Vaduvescu et al. (2007) calculated via K-band photometry (we use the latter values in our Fig. 11). We see that Virgo dwarf galaxies couple nicely with our Hercules data on the MZ plot. We also compare with the extensive sample of SF galaxies used by Amorín et al. (2010), comprised by all the emission-line galaxies listed in the Max Planck Institute for Astrophysics/Johns Hopkings University (MPA/JHU) Data catalog of the SDSS DR 713 which covers a redshift range $0.03 \leq z \leq 0.37$. Oxygen abundances were calculated using the N2 calibration of PM09 and mass estimates were driven from

\footnotetext{
${ }^{13}$ Available at http://www.mpa-garching.mpg.de/SDSS/
}

the MPA/JHU catalog. On this plot Hercules SF galaxies lie within the same range as the emissionline galaxies of SDSS DR 7, except in the higher mass range, where our data show a shift towards lower metallicities by $\sim 0.15 \mathrm{dex}$ as compared to the Amorín et al. (2010) sample. We tentatively attribute this observed shift to the different oxygen abundance calibration used in each case; in addition, another important effect is the different spatial coverage of SDSS spectra versus the spatially resolved spectra used in this work.

In Fig. 12 (left) we plot the gas-phase oxygen abundance versus galaxy $\mathrm{M}_{\mathrm{B}}$ absolute magnitude. Our galaxy sample follows a LZ relation, where the same differential behavior identified in the MZ relation is clear for the dwarf/irregular galaxies at high local densities (i.e. $\log \Sigma_{4,5}>1.85$ ). On the plot to the right we show again the Virgo dI and BCDs (light blue open circles) (Vílchez \& Iglesias-Páramo 2003; Vaduvescu et al. 2007), and the (centers of) Virgo spirals (red open triangles) from Pilyugin et al. (2002) and references therein. We also add the Hydra dwarfs (green open diamonds) from Duc et al. (2001, 1999). All the abundances were recalculated using P10 method and $\mathrm{M}_{\mathrm{B}}$ of the Virgo spirals was derived by the $L_{B}$ given by Skillman et al. (1996). We see that SF galaxies of Hydra, Virgo, and Hercules clusters appear to follow the overall LZ relation, though is apparent that the scatter has increased.

Moreover, Hercules SF galaxies also follow a well defined sequence on the plots of the $\mathrm{N} / \mathrm{O}$ ratio versus galaxy stellar mass and galaxy $\mathrm{M}_{\mathrm{B}}$ absolute magnitude shown in Fig. 13. In this plot we can see how some of the dwarf galaxies shifted towards higher $\mathrm{O}$ values in the MZ relation appear overabundant in $\mathrm{N} / \mathrm{O}$ (see also $\$ 5.3 .2)$. Additionally, the $\mathrm{N} / \mathrm{O}$ ratio makes evident a significant abundance difference between nuclei and disks (points connected with straight lines), that will be discussed in more detail in $\$ 5.4$.

Very recent works (Mannucci et al. 2010; Lara-López et al. 2010) discuss the existence of a more general relation between stellar mass, gas-phase metallicity and star formation rate in the local universe. We have checked that, for the galaxy mass range we have in common, Hercules SF galaxies lie in the same range as Lara-López et al. (2010) sample in the $3 \mathrm{D}$ plot which combines stellar mass, gas- 

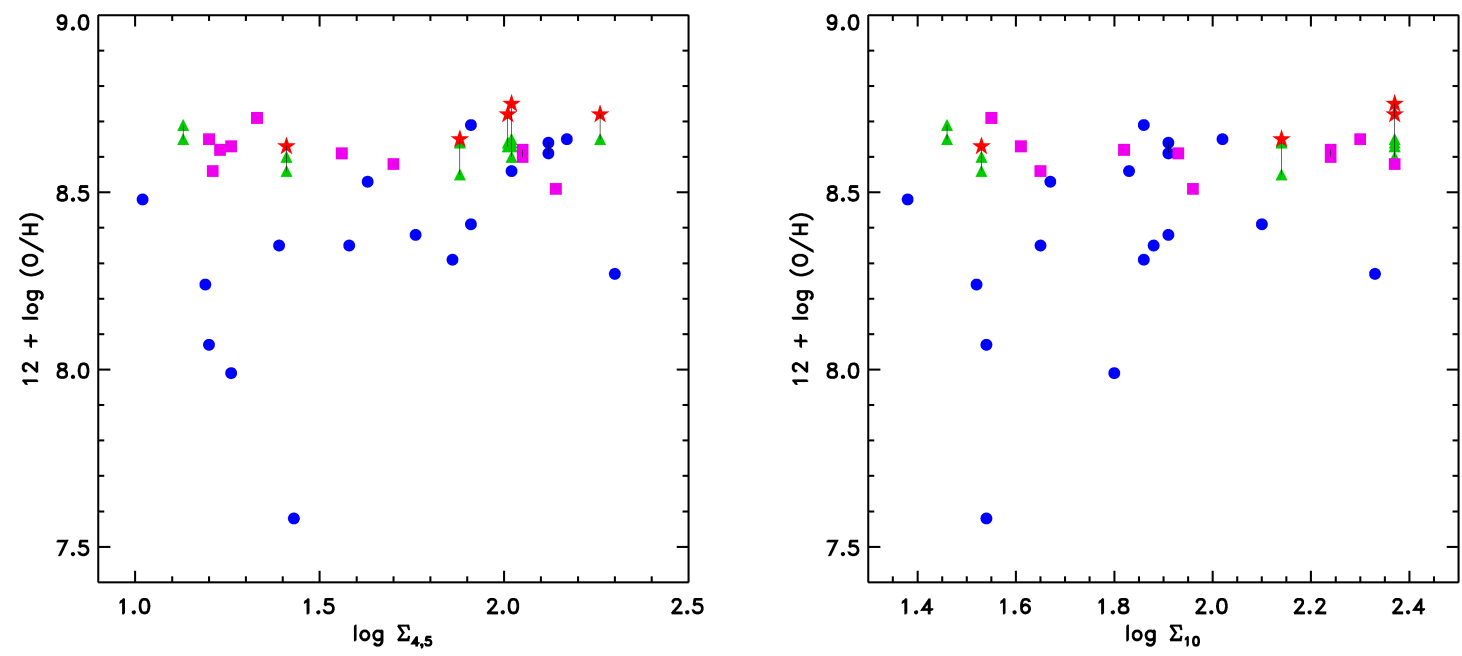

Fig. 10.- Oxygen abundance of galaxies versus their local density. Left: $\mathrm{O} / \mathrm{H}$ vs $\Sigma_{4,5}$, the local galaxy number density to the average of the projected distances to the fourth and fifth nearest neighbor, where the galaxies considered are secure members of the Hercules cluster, as follows by their SDSS spectroscopic redshift. Right: $\mathrm{O} / \mathrm{H}$ vs $\Sigma_{10}$, the local galaxy number density to the $10^{\text {th }}$ nearest neighbor, where a magnitude limited sample $\left(\mathrm{M}_{\mathrm{r}^{\prime}} \leq-19 \mathrm{mag}\right)$ of galaxies is used and we remedy for background and foreground galaxies using the field galaxy counts of Yasuda et al. (2001). Colors and point features as in Fig. 11 blue filled circles for dwarf/irregular galaxies $\left(\mathrm{M}_{\mathrm{B}}>-19\right)$, magenta squares for spirals $\left(\mathrm{M}_{\mathrm{B}} \leq-19\right)$ integrated galaxies, red stars for the nuclei of 6 spirals that we divide into different parts and green triangles for their corresponding disk components (nuclei and disks are connected with lines). Both parts of PGC057077 are plotted in magenta filled squares; similarly, both parts of PGC057064 are plotted in blue filled circles. 
phase metallicity and star formation rate. However, in this work we prefer not extending this discussion until our spectroscopic study of several nearby clusters is finished (Petropoulou et al. in prep), where metallicities, stellar masses, and SFR are computed for a large sample of SF cluster galaxies. With this new dataset a study of the fundamental plane for SF galaxies in clusters could be performed.

\subsection{Environment and chemical enrich- ment in Hercules galaxies}

\subsubsection{Comparison with the closed-box model}

In order to study possible environmental effects on the (gas-phase) chemical enrichment in galaxies, it is useful to compare with the predictions of the so-called closed-box model (Edmunds 1990). According to this model, a galaxy consists initially of gas with no stars and no metals. The stellar IMF is assumed to be constant on time and the products of stellar nucleosynthesis are assumed to enrich the ISM instantaneously. Throughout its life, the metal content of a galaxy is neither diluted by infalling pristine gas nor lost via outflow of enriched gas. Hence, the metallicity at any given time is only determined by the fraction of baryons which remains in gaseous form. The model equation can be written as

$$
Z_{o}=y_{o} \ln (1 / \mu)
$$

where $Z_{o}$ is the oxygen mass fraction, $y_{o}$ is the yield by mass and $\mu$ is the ratio of the gas mass to the baryonic mass, $\mu=M_{g a s} / M_{\text {bar }}$. The gas mass corresponds to the hydrogen atomic gas with a correction for neutral helium $\left(M_{\text {gas }}=1.32 M_{H I}\right.$; molecular gas mass was not taken into account here) and $M_{b a r}=M_{g a s}+M_{\star}$ where $M_{\star}$ is the mass in stars.

Fig. 14 compares the observed oxygen abundances for our sample galaxies to the prediction of the closed-box model, plotted with lines with different $y_{o}$. The green continuous line indicates the model with $y_{o}=0.0074$, that is the theoretical yield of oxygen expected for a Salpeter IMF and constant star formation rate, for stars with rotation following Mevnet \& Maeder (2002) models (van Zee \& Haynes 2006). Both, infall and outflow of well-mixed material will result in effective yields that are less than the true yields, as the enriched material is either diluted (infall) or lost from the system (outflow). A fraction of the sample of isolated dwarf irregular (dI) galaxies of van Zee \& Haynes (2006) was found to follow the theoretical yield, and the rest appears consistent with a lower yield $y_{o}=0.002$, almost $1 / 4$ of the model prediction (blue dashed line in Fig. 14). The gray strip indicates the relationship given by Lee et al. (2003) between oxygen abundance and the baryonic gas fraction for a sample of local Universe dIs. The cyan open circles correspond to the Virgo dI and BCDs from Vaduvescu et al. (2007). Most of Virgo dwarfs appear consistent with the Lee et al. (2003) locus for local dwarf galaxies, though some of them still present lower gas fractions.

We can see in Fig. 14 that four of our low mass galaxies for which we have HI measurements (Leda1543586, LEDA140568, [D97]ce-200, and LEDA3085054), are in agreement with the closed-box model predictions with $y_{o}=0.0074$ (green line). This fact suggests that these galaxies are falling into the central region of the cluster and encountering now the dense ICM gas for the first time; as a consequence, gas removal by rampressure stripping might not yet be observable. These four galaxies can be considered as prototypes of the "newcomers" to the cluster introduced in 5.1 This result is additionally supported by their disturbed $\mathrm{H} \alpha$ characteristic morphologies (as seen in the $\mathrm{H} \alpha$ maps in $\mathrm{C} 09$ ) as described below. The $\mathrm{H} \alpha$ emission map of Leda1543586 reveals a strong episode of star formation concentrated into an asymmetric arc located on the side of the galaxy facing the cluster center and almost no emission in the opposite side. This typical "bow" morphology has been observed in other cluster galaxies (e.g. Gavazzi et al. 2001) and is very suggestive of a ram-pressure event. After closer inspection of the $\mathrm{C} 09 \mathrm{H} \alpha$ maps we identify that the galaxy LEDA140568 also show a "bowshock" morphology on the side of the galaxy facing the cluster center. Additionally, the other 2 galaxies, [D97]ce-200, and LEDA3085054 present strongly asymmetric $\mathrm{H} \alpha$ emission, one-sided and offset from the galaxy optical center. These starbursts could be the signature of pressure-triggered star formation by the ICM within the cluster environment (Treu et al. 2003). Additionally these four galaxies have $\operatorname{abs}(\Delta \mathrm{V})>\sigma_{\mathrm{V}}$ (see $2.1 \mathrm{~d}$ ) and 

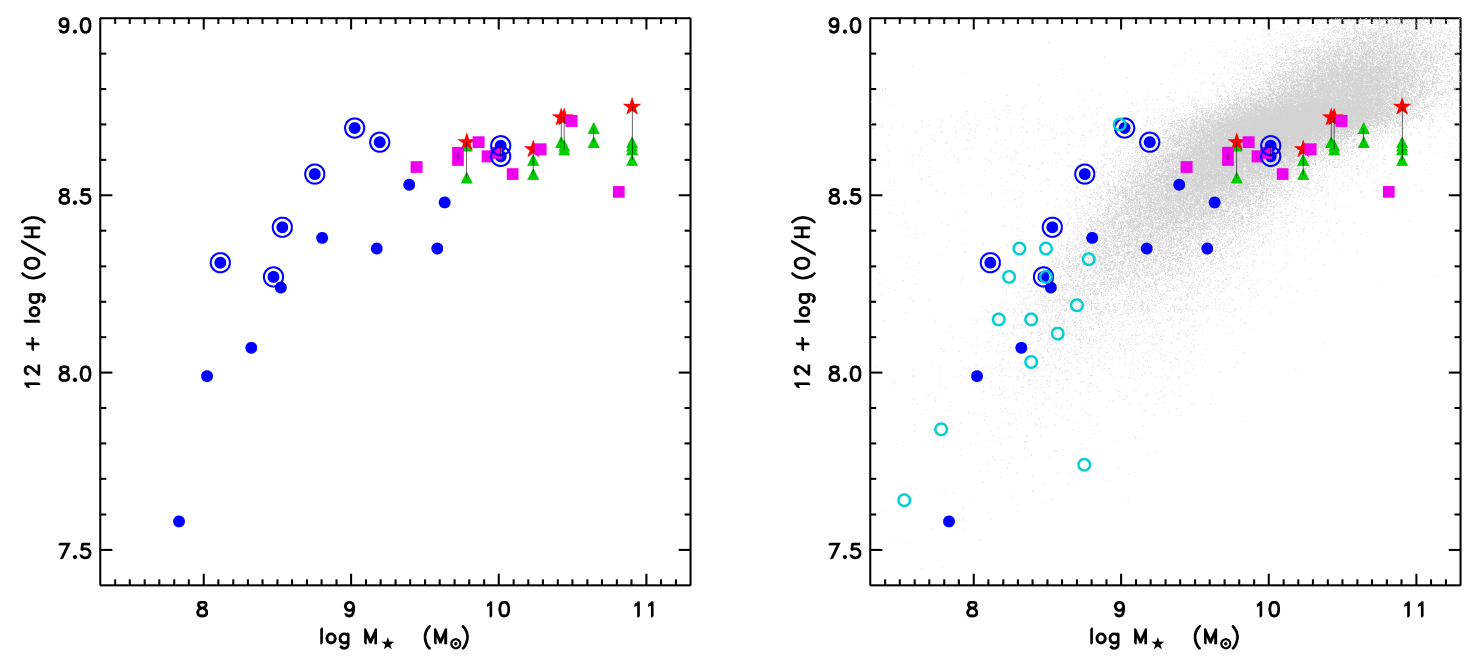

Fig. 11.- Left: Gas-phase oxygen abundance vs galaxy stellar mass for the Hercules galaxies. We keep the same color distinction as in Fig. 10. We mark with circles the galaxies located at densities $\log \Sigma_{4,5}>1.85$ which appear preferentially located above the general relation. Right: On the same plot we add the Virgo dIs and BCDs from Vaduvescu et al. (2007) (light blue open circles) and the MPA/JHU SF galaxies (gray points) used as a reference sample by Amorín et al. (2010).
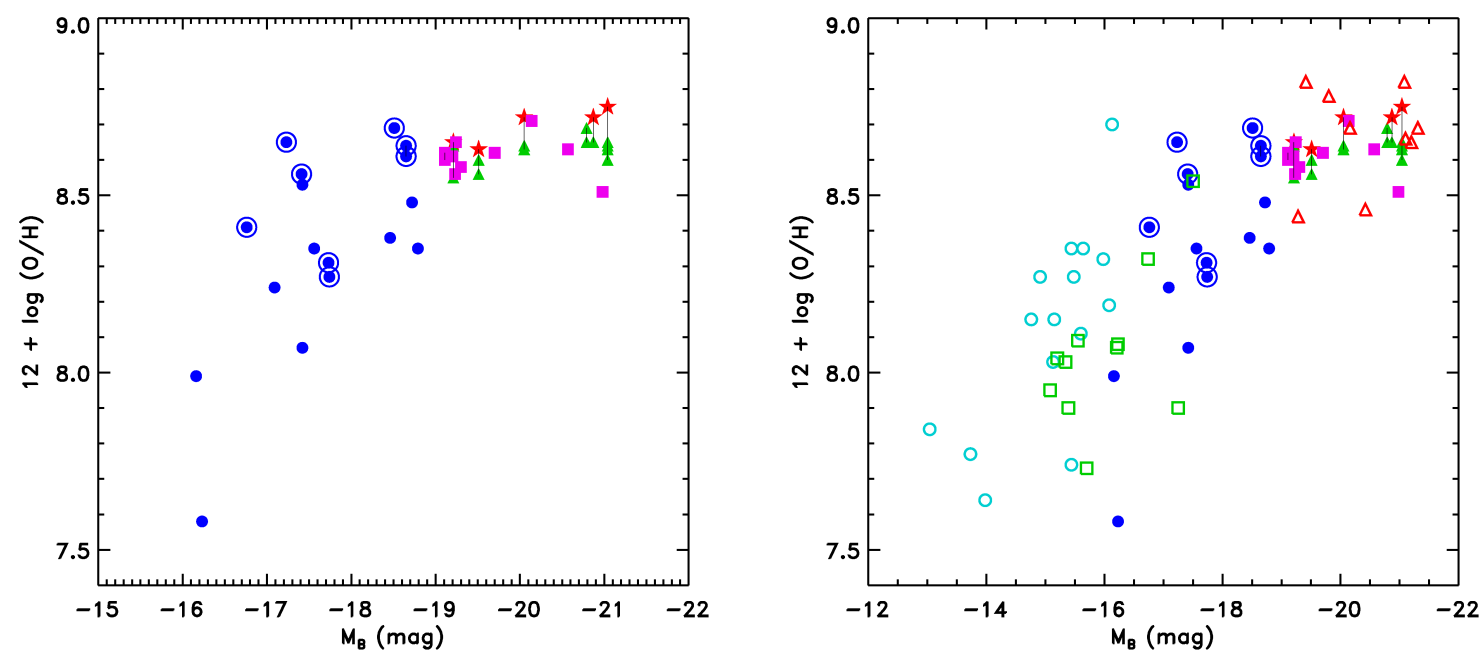

Fig. 12.- Left: Gas-phase oxygen abundance vs galaxy $\mathrm{M}_{\mathrm{B}}$ absolute magnitude for the Hercules sample (colors and symbols as in Fig. 11). As in the MZ relation, galaxies (marked with circles) at high density $\left(\log \Sigma_{4,5}>1.85\right)$ appear shifted towards higher $\mathrm{O} / \mathrm{H}$. Right: We add the Virgo dI and BCDs (light blue circles) as given by Vílchez \& Iglesias-Páramo (2003) and Vaduvescu et al. (2007), the Virgo spirals (central abundance; red open triangles) from Pilvugin et al. (2002) and references therein, as well as the Hydra dwarfs (green open squares) by Duc et al. (2001, 1999). 

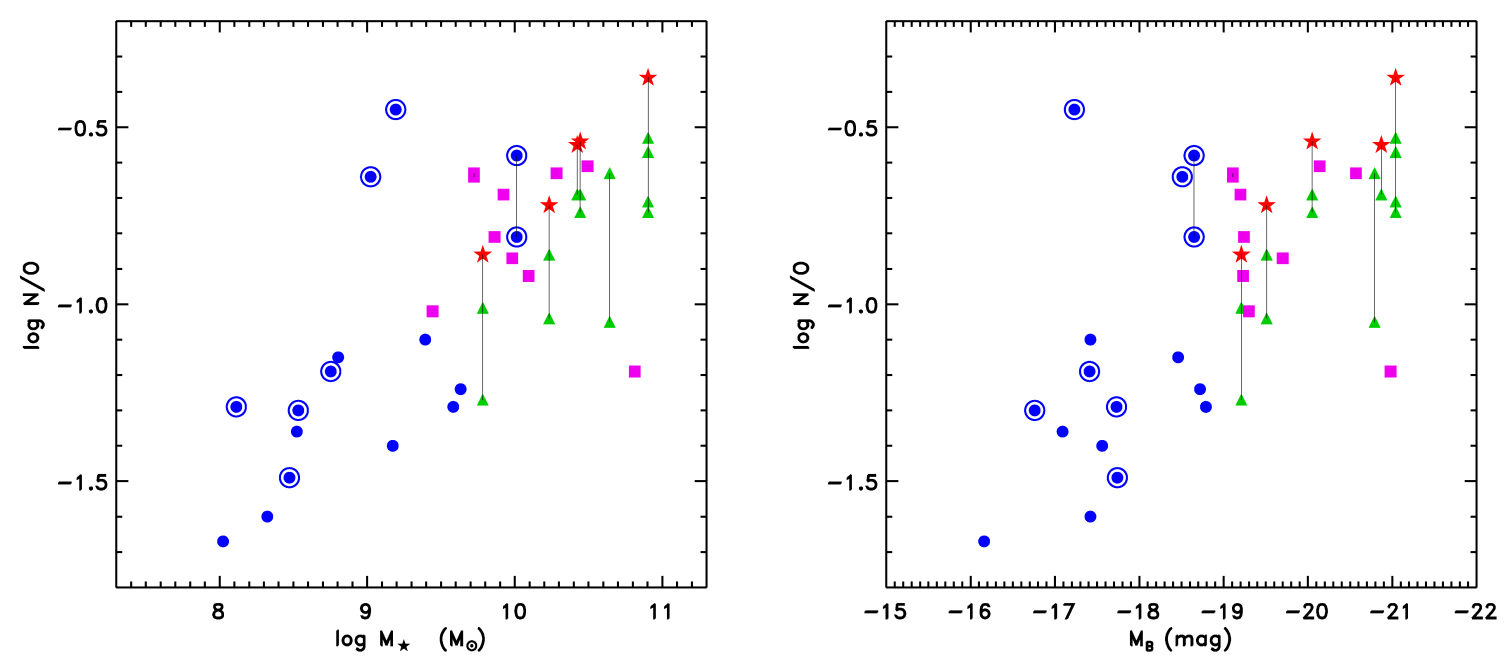

Fig. 13. - N/O vs galaxy stellar mass (left) and $\mathrm{M}_{\mathrm{B}}$ absolute magnitude (right) for the Hercules galaxies (colors and symbols as in Fig. 11). A significant $\mathrm{N} / \mathrm{O}$ abundance difference can be seen between galaxy nuclei and disks (points connected with straight lines).

they have a median projected distance to the center of the X-ray distribution of $\sim 700 \mathrm{kpc}$; thus they are approaching to the edge of the main $\mathrm{X}$ ray emitting region which extends up to $R_{X}=678$ kpc (Huang \& Sarazin 1996).

The blue points close to the blue dashed line in Fig. 14 $\left(y_{o}=0.002\right)$ correspond to the dwarf/irregular galaxies [D97]ce-143 and LEDA084703 (see the Appendix for details). [D97]ce-143 is located at high local density (\$5.1) and Dickey (1997) reports that its correspondent HI cloud shows two elongations and is highly reminiscent of the Magellanic Stream. LEDA084703 shows a long HI plume (Dickey 1997) reaching about $1^{\prime}$ from the optical center to the southeast (also at the east part of this galaxy is located the supernova quoted by Zwicky et al. 1969). These peculiar HI morphologies suggest that some gas mass-loss effect has taken place, explaining thus their location on Fig. 14

A very interesting case is the galaxy IC1182:[S72]d labeled in Fig. 14. This tidal dwarf candidate (TDC, Iglesias-Páramo et al. 2003) shows an HI distribution which extends well beyond the galaxy disk (Dickey 1997). This morphology, combined with the information we got on its gas-phase chemical content (this galaxy lies above the MZ and mass-N/O relations), together with its old stellar population (see \$5.3.2), indicate a particular formation scenario for this galaxy. This galaxy has probably been the result of a "block" produced during the merger IC1182 and seems dominated by an old stellar population. This stellar "block" could have acquired a large mass of gas from the late type galaxy of the merger IC1182. This formation scenario can explain why IC1182:[S72]d shows a much higher gas fraction than the value expected according to the closedbox model. Thus, the active environment of the cluster can provide an explanation for the location of a galaxy in such forbidden region on Fig. 14 (Edmunds 1990; van Zee \& Haynes 2006). There exist examples of dwarf galaxies formed from the gas lost in a merger, e.g. the old TDG VCC 2062 in the Virgo cluster studied by Duc et al. (2007); according to these authors this galaxy has probably been formed out of the gas clouds lost by a gas-rich galaxy involved in a merger.

For the galaxies of our sample that were not detected in Dickev (1997) we assume an HI mass upper limit corresponding to the detection threshold of this survey $\left(\leq 2.6 \times 10^{8} M_{\odot}\right)$. In Fig. 14 we add those galaxies with right pointing arrows (representative of the central oxygen abundance) to indicate the upper limit for their HI mass. Excluding the galaxies already discussed, the rest of 
the galaxies in Fig. 14 (including upper limits) on average suggest effective yields below the closed box model and are consistent with the field sample of Lee et al. (2003). Finally, some points (few Virgo dwarfs and one Hercules upper limit for the IC1182 merger) still appear in Fig 14 displaced towards even lower values of gas fractions, as it would be expected if these cluster galaxies suffered important environmentally induced gas removal (e.g. from ram-pressure stripping).

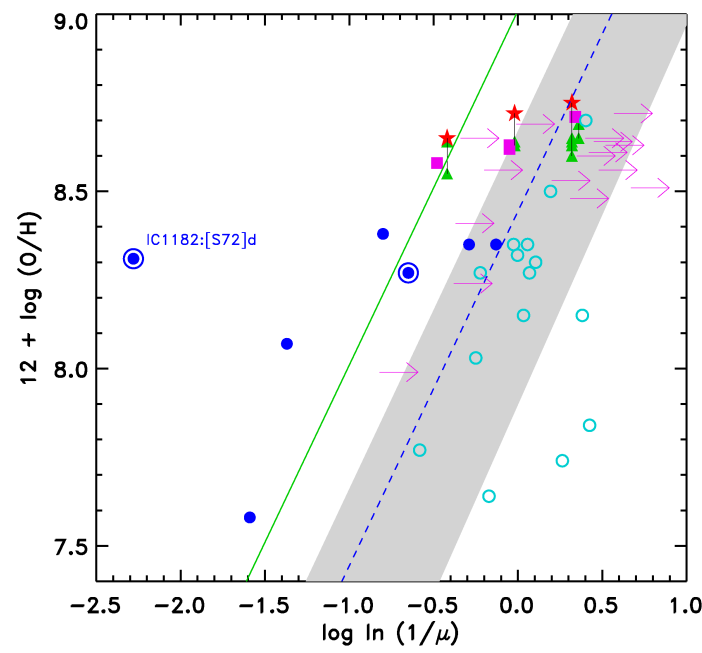

Fig. 14.- Oxygen abundances for our sample galaxies vs the ratio of the gas mass to the baryonic mass, $\mu=M_{\text {gas }} / M_{\text {bar }}$ (colors and symbols as in Fig. 11). The green continuous line indicates the closed-box model with $y_{0}=0.0074$ and the blue dashed line corresponds to $y_{0}=0.002$. The gray strip gives the best fit of Lee et al. (2003) to their field dIs. The cyan circles correspond to the Virgo dI and BCDs from Vaduvescu et al. (2007). Arrows mark objects with upper limit in HI.

\subsubsection{Gas-phase metallicity vs properties of the underlying stellar component}

In order to search for possible environmental footprints on the chemical history of our sample galaxies, we compare the stellar population properties, such as the mass-weighted stellar metallicity $Z_{\star, M}$ and mass-weighted stellar age $\tau_{\star, M}$, brought forth by the STARLIGHT model fitting (\$3), with the gas-phase abundances derived in this work. The fact that these properties (for stars and gas) have been obtained following a completely different methodology should render our analysis more robust.

Fig. 15] shows the gas-phase oxygen abundance (left) and the N/O ratio (right) versus the massweighted stellar age $\tau_{\star, M}$. We see an overall positive trend, more prominent for $\mathrm{N} / \mathrm{O}$, which should reflect the different time-scales for the delivery of these two elements to the ISM. Oxygen, produced in Type II supernovae, is released after $\sim 10 \mathrm{Myr}$, while nitrogen is produced and released over a substantially longer period, $\gtrsim 250 \mathrm{Myr}$. Overall, we can see how nitrogen abundance seems to correlate better with the mass-weighted stellar age. Additionally, the dispersion in N/O becomes smaller at larger age, possibly reflecting the averaging effects of many SF episodes, while the larger dispersion seen at small ages could reflect the stochastic effects of few episodes of SF or other possible environmental effects such as e.g. gas inflows.

In Fig. [15] we can see that $\gtrsim 85 \%$ of the dwarf galaxies residing at high local density environments $\left(\log \Sigma_{4,5}>1.85\right)$ present old stellar populations of mass weighted age $\geqslant 6$ Gyr. Conversely, $\sim 70 \%$ of the dwarfs located at density $\log \Sigma_{4,5}<1.85$ present mass weighted ages below this value; a hint suggesting that only the more robust galaxies - e.g. more massive, evolved and more metallic- could have survived in the environment of highest galaxy density. The outliers to this general correlation also can give us important clues on possible environmental effects on their chemical histories. On the right plot of Fig. 15 we can identify the galaxy merger IC1182 and the two dwarfs associated to it (SDSS J150531.84+174826.1 and IC1182:[S72]d; see Appendix). Interestingly enough, all three objects present almost the same (very old) stellar age. Moreover, the galaxy SDSS J150531.84+174826.1 appears to be more chemically enriched with respect to galaxies of similar mass (see also Figs. 11 and 13). Taken altogether, these properties of SDSS J150531.84+174826.1 remind those of the central part of a massive galaxy after having lost its outer parts (e.g. during a past interaction with the neighbor merger IC1182). The TDC galaxy IC1182:[S72]d, despite hosting an old stellar population, does not seem to be that chemically evolved (especially in $\mathrm{N} / \mathrm{O}$ ) in accordance with the scenario already proposed for it: the accretion of a 
large mass of gas from the late type galaxy of the merger IC1182. For IC1182, our spectrum sampled the slightly off-center starburst giving a gaseous oxygen abundance slightly lower than the average observed trend in the MZ relation. This fact, together with its low N/O ratio, suggest that this gas should have an origin external to the host underlying galaxy. A similar scenario has been invoked before by Moles et al. (2004) (see also Radovich et al. 2005) who claimed that this gas was provided by the late type galaxy of the merger. A detailed study of this complex system is out of the scope of this work and will be presented in a forthcoming paper (Petropoulou et al, in prep.).

Two other objects can be seen above the correlation in Fig. 15. the eastern part of the disk of NGC6045 (NGC6045e) and PGC057077b, both galaxies appear affected by interactions and show intense SF (the highest SFR values measured for our sample after the merger IC1182). Though it has been shown that high SFR can be a key ingredient to enhance N/O (Mollá et al. 2006), what moves these two objects out of the general age$\mathrm{N} / \mathrm{O}$ relation seems to be related to their derived ages: the spectra of these objects are sampling just the starbursts, hence the derivation of $\tau_{\star, M}$ should then be dominated by the contribution of the young starburst; in contrast, the results derived for other parts of these two galaxies follow the general trend of $\mathrm{N} / \mathrm{O}$ vs $\tau_{\star, M}$.

In Fig. [16 we compare the gas-phase and the stellar oxygen abundances derived for the sample of galaxies. We compute the latter abundance using the mass-weighted metallicity, $Z_{\star, M}$, given by STARLIGHT, assuming $12+\log (\mathrm{O} / \mathrm{H})_{\odot}=8.69$ (Asplund et al. 2009). We plot the difference between gas-phase and stellar oxygen abundance $\Delta \log (\mathrm{O} / \mathrm{H})_{\text {gas }-\star}$ versus the stellar oxygen abundance $12+\log (\mathrm{O} / \mathrm{H})_{\star}$. In this plot we can see two main behaviors. For luminous galaxies $\left(\mathrm{M}_{\mathrm{B}} \leq\right.$ -19), we can see the following correlation:

$$
y=(7.99 \pm 0.47)-(0.925 \pm 0.055) x
$$

where $y=\Delta \log (O / H)_{\text {gas }-\star}$ and $x=12+$ $\log (O / H)_{\star}\left(\chi^{2}=0.074\right)$. Assuming our model fitting hypotheses, this "upper bound" correlation would mean that for a chemically evolved stellar population, of order $\sim Z_{\odot}$, gas and stars present the same metallicity. However, even when the stellar metal content of these galaxies goes down, we can see the observed gas abundance remains close to $\sim Z_{\odot}$ (see also Gallazzi et al. 2005; Asari et al. 2007). This behavior could reflect the fact that, for our more massive galaxies, gradients of stellar metallicity can be more conspicuous than for gas, consistent with model predictions (Ferrini et al. 1994) suggesting that abundance gradients should flatten with age.

For the lower luminosity galaxies we can see that the two groups of dwarfs (referred to in 55.2 (5.3.1) split up in Fig 16 too. The "newcomers" show similar oxygen abundances for gas and stars whereas the more chemically evolved dwarfs found at high local densities (marked with circles), hosting older stellar populations ( $\geqslant 6 \mathrm{Gyr}$ ), show that the gas oxygen abundance is higher than the abundance of the stars by up to 0.3 dex. An outlier to this relation, given its peculiar formation already unraveled, is the galaxy IC1182:[S72]d, for which the derived stellar metallicity is higher than the gaseous one. Whether these two behaviors, shown in Fig. 16. result from different chemical evolutionary paths or rather reflect the environmental impact of the cluster remains to be disentangled.

\subsection{Searching for the cluster influence in Hercules SF galaxies}

A considerable amount of work has been done in order to constrain the physical mechanisms that drive the SFH of galaxies, these mechanisms being either internal to the galaxy or related to the environment (Haines et al. 2007; Bretherton et al. 2010; Weisz et al. 2011). In the cluster environment it has been found that quenching mechanisms can even suppress the SF of galaxies with respect to their field counterparts and morphological changes can operate converting spirals into anemic (Balogh et al. 2004). Various processes have been proposed to describe the environmental actions on cluster galaxies, these can be classified into three broad categories: 1) galaxy-ICM interactions, 2) galaxy-cluster gravitational interactions, 3) galaxy-galaxy interactions (for a review see e.g. Boselli \& Gavazzi 2006; Treu et al. 2003).

In this work we have searched for possible observable imprints of the cluster environment on the metallicity and the chemical history of a sample of SF galaxies in the Hercules cluster. Most of our sample galaxies are located within the central 

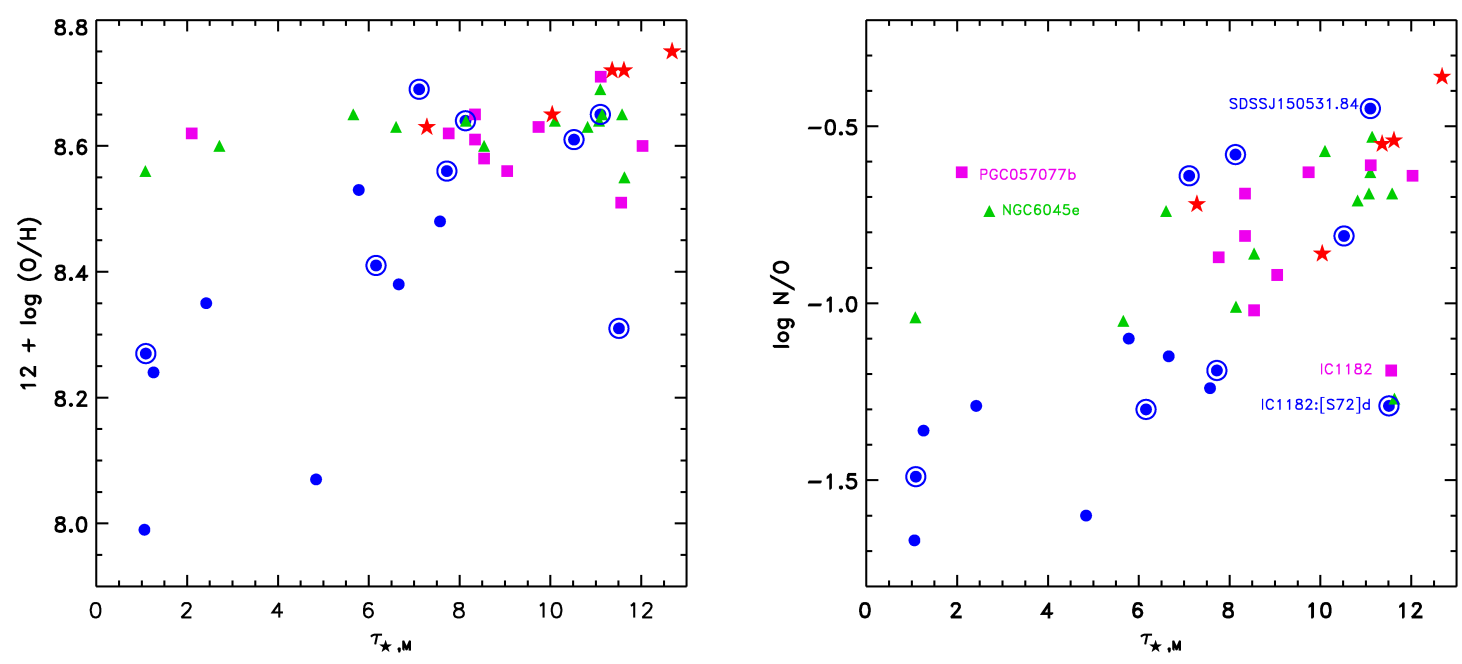

Fig. 15. - The gas-phase oxygen abundance (left) and N/O ratio (right) versus the mass-weighted stellar age $\tau_{\star, M}$ as given by STARLIGHT model fitting (colors and symbols as in Fig. 11). An overall positive trend can be seen (see the text for details).

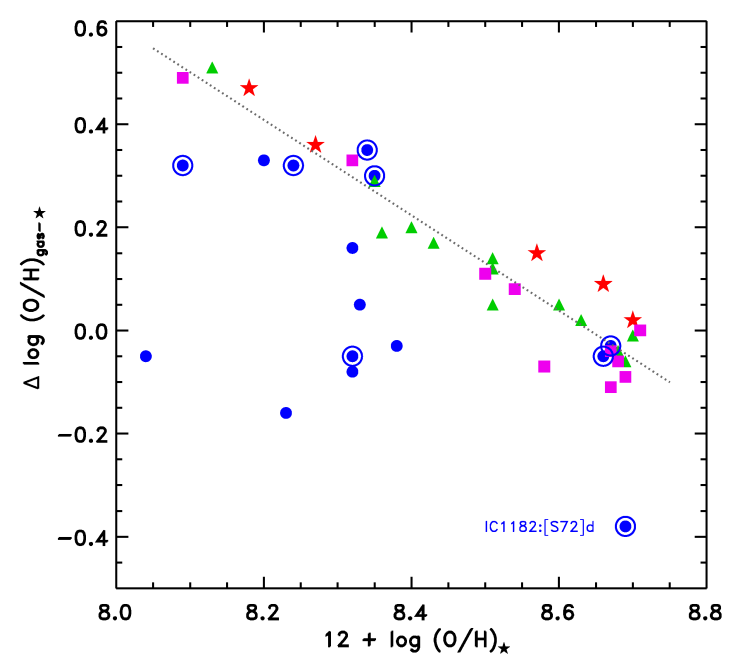

Fig. 16. - The difference between gas-phase and stellar oxygen abundances versus stellar oxygen abundance $12+\log (O / H)_{\star}$ derived from the STARLIGHT mass-weighted stellar metallicity $Z_{\star, m}$ (colors and symbols as in Fig. 11). region of the Hercules cluster $\left(<R_{200}\right)$ where the cluster potential is expected to be steep, the ICM is measurable and most of the physical mechanisms proposed above are effectively at work. We have examined the metal content of our sample galaxies as a function of local density, stellar mass, luminosity and chemical evolution. On the basis of this analysis, three main subgroups of objects have emerged: 1) the more massive SF galaxies (including all spirals), 2) the set of dwarf/irregulars labeled "newcomers" in 55.3 .1 and 3) a group of more chemically evolved dwarfs residing at the highest local densities observed in our sample. In addition, three dwarf/irregular galaxies SDSS J160524.27+175329.3, SDSS J160556.98+174304.1 and LEDA084724, could not be fully adscribed to any of these groups (see the Appendix).

Massive galaxies $\left(M_{B} \leq-19\right)$ follow the global MZ/LZ relations, though they present evidences of being affected by the cluster environment, judging from their $\mathrm{H} \alpha$ structure/morphology and their abundance gradients. Most spirals in Hercules show $\mathrm{H} \alpha$ emission (see $\mathrm{H} \alpha$ maps in C09) less extended than their optical disks and sometimes offset from the center of the stellar continuum. This can be an evidence for truncation of ionized gas in the disks of these cluster spirals, since we observe star-formation occurring mostly in the inner parts. 
Gas removal from the outer parts of cluster spirals is believed to be a consequence of the ISM-ICM interaction. This effect has been observed e.g. in the Virgo cluster spirals by Koopmann \& Kenney (2004), where over half of their Virgo sample show truncation of the star-formation in the disks. In fact, ram-pressure gas stripping could be effective on our Hercules spirals since all are located within $R_{200}$.

The oxygen abundances derived for massive galaxies are close to solar, and for the more $\mathrm{H} \alpha$ extended spirals, mild or flat $\mathrm{O} / \mathrm{H}$ abundance gradients have been obtained, a result in the line of previous findings by Skillman et al. (1996) for Virgo spirals. In contrast, for the $\mathrm{N} / \mathrm{O}$ ratio even oversolar values have been measured for the central part of some galaxies, showing prominent $\mathrm{N} / \mathrm{O}$ spatial variations; this picture could result from the effect of gas infall in the center of these galaxies, suffering the action of the ICM (Vollmer et al. 2001). Such infall would dilute the abundance at the central parts of the galaxies flattening the $\mathrm{O} / \mathrm{H}$ gradient, while the $\mathrm{N} / \mathrm{O}$ ratio is not expected to be affected. Overall, the question which remains to be explored is whether these spirals are chemically evolved because they reside in such high density environments or we are just observing an effect of the morphology density relation (Dressler 1980). Further observations are needed to answer this question.

Regarding the dwarf/irregular galaxies, they appear to form two main groups with substantial differences as it has emerged in the previous sections. Overall, all the dwarfs present similar levels of SFR as derived from their $\mathrm{H} \alpha$ luminosities. The group of "newcomers" are metal poor dwarfs, they present a young stellar population (Fig. 15), and their stellar and gas metallicities are similar (Fig. 16). They avoid the highest local densities (\$5.1), appear located close to the boundaries of the X-ray cluster core and show bowshock/offset $\mathrm{H} \alpha$ morphologies. These structures host intense bursts of SF, possibly tracing the contact discontinuity of the ISM of the galaxy with the X-ray emitting ICM, an observable signature of pressure-triggered star formation. Though, the "newcomers" follow the closed-box model predictions, suggesting that ram-pressure stripping has not yet substantially reduced their HI gas. Rampressure stripping is expected to act on a time- scale $\sim 5 \times 10^{7}$ yr (Abadi et al. 1999), while typical $\mathrm{H}$ II regions lifetimes are $\sim 5-10 \times 10^{6} \mathrm{yr}$. Based on this scheme we should conclude that the "newcomers" are those dwarfs observed right on time when they are set to fire by their first encounter with the ICM; before removal of the galactic gas is accomplished by the ICM and prior to subsequent quenching of SF rendering them undetectable in $\mathrm{H} \alpha$.

The majority of the dwarf/irregulars populating the highest local densities of our sample $\left(\log \Sigma_{4,5}>1.85\right)$ show higher metallicities for their mass and luminosity and they appear located above the overall MZ and LZ relations (see Figs.1112). Their gaseous and stellar abundances differ by up to $\sim 0.3$ dex, pointing towards a dominant old stellar population. Indeed, as seen in Figs. 15 their stellar population presents ages exceeding $\sim 6$ Gyr. These galaxies have been also found close to the X-ray cluster core, but their $\mathrm{H} \alpha$ morphologies do not suggest ISM-ICM interaction. However we can not discard they could have been possibly affected by ram-pressure stripping. Although HI masses are not available for the vast majority of these objects, if we assume an HI mass upper limit for them as in \$5.3.1 many of these galaxies would be shifted in Fig. 14 out of the canonical closed-box model, towards the zone of HI deficiency.

We have seen that high local density is a key parameter that separates this group of chemically evolved dwarfs from the rest of the dwarfs. At these high density environments preprocessing has been claimed to operate under the combined action of tidal forces among group members and the ram-pressure by the ICM (Cortese et al. 2006). We suggest that these dwarf galaxies over-metallic for their mass-could originate from enriched material stripped by tidal forces among group members, as it has been suggested for the group of galaxies falling into Abell 1367 by Cortese et al. (2006). Indeed we have identified a good number of these more metallic dwarfs affected by tidal interactions.

Mahajan et al. (2011) have found two sets of blue dwarf galaxies with different $\mathrm{H} \alpha$ emission properties in the Coma supercluster, presenting strong environmental dependence. These authors suggest that the more evolved dwarf population could be the progenitors of passive dwarf galax- 
ies seen in the centers of clusters. The two dwarf galaxy groups we have identified in Hercules could match this scheme.

In this work we have studied SF galaxies located in the cluster central region $\left(<R_{200}\right)$; as a consequence the discussion on the physical mechanisms affecting galaxies in the cluster environment is restricted to $\mathrm{SF}$ galaxies and it could not have been exhaustive by no means. It would be necessary a larger sample covering a more extended area of the cluster in order to explore the full action of other processes able to suppress SF, like starvation of the gaseous component, harassment, or interactions with the global cluster potential. An extended $\mathrm{H} \alpha$ survey reaching up to the Hercules cluster periphery is in progress. This will enable us to study in depth the interesting general environment of the Hercules cluster. Bird et al. (1995) using optical and X-ray data suggested the presence of at least three distinct subclusters in Hercules cluster, the central A2151C, eastern A2151E and northern A2151N, see also Fig. 2. These authors suggested that the A2151E and A2151N subcluster have recently undergone a merger event. Additionally, the velocity distribution of A2151C points towards the existence of two subgroups, one possibly originating from A2151N via infall. The $\mathrm{X}$-ray emission is associated with the two galaxy groups in the central subcluster (Fig. 22). All this information supports the idea that Hercules is at a relatively early stage of development.

\section{SUMMARY}

The Hercules cluster is one of the most exciting nearby dense environments, showing abundant sub-structures unraveled in X-ray emission and broadband imaging. This cluster constitutes an ideal laboratory to explore the effects of the environment on galaxy evolution. We have studied the environmental effects on the metallicity and the chemical evolution of $31 \mathrm{SF}$ cluster galaxies.

Spatially resolved spectroscopy has been obtained for a sample of SF galaxies and spectral synthesis model fitting has been performed for all the spectra analyzed in order to provide an effective correction of the underlying stellar absorption on emission line spectra, as well as to derive the characteristic properties of the galaxy stellar populations. Line fluxes and chemical abundances of $\mathrm{O} / \mathrm{H}$ and $\mathrm{N} / \mathrm{O}$ have been obtained for all the galaxies, and whenever possible for different part of galaxies, of the sample.

The main conclusions of this work can be summarized as follows:

1) From the study of the metallicity vs. galaxy local density we have seen a dual behavior separating the dwarfs from the more luminous galaxies. The luminous galaxies have metallicities $\sim Z_{\odot}$ and reside at all densities studied in this work. The set of dwarfs found at higher local densities $\left(\log \Sigma_{4,5}>1.8\right)$ are found to be more metallic $(12+\log (\mathrm{O} / \mathrm{H})>8.4)$ while the observed less metallic dwarfs $(12+\log (\mathrm{O} / \mathrm{H})<8.4$ are found preferentially at lower densities and some of them seem to be "newcomers" to the cluster.

2) We have found that our sample of Hercules $\mathrm{SF}$ galaxies shows well defined sequences of blue luminosity vs. metallicity and stellar mass vs. metallicity (using both $\mathrm{O} / \mathrm{H}$ and also the $\mathrm{N} / \mathrm{O}$ ratio), following the general behavior found for $\mathrm{SF}$ galaxies. Besides this global behavior, we have found that dwarf/irregular galaxies populating the densest regions seem to crowd the upper part of the global sequences, thus providing a source of the dispersion observed in these relations. These more metallic dwarfs could be parts of more massive galaxies, fragmented by tidal interactions among group members.

3) Most of the luminous galaxies are chemically evolved spirals with oxygen abundance close to solar and truncated disks of ionized gas, possibly by the action of ram-pressure stripping. From our spatially resolved spectroscopy we have found that the $\mathrm{H} \alpha$ extended spiral galaxies present shallow oxygen abundance gradients, an expected result of possible gas infall at their centers. For the N/O ratio, even oversolar values have been obtained for the central parts of some galaxies and a significant spatial variation has been observed.

4) A detailed study of the chemical history of the sample galaxies has been performed, combining information on their gas-phase abundances, HI content and stellar mass. Most of the dwarf galaxies with available HI mass seem to be "newcomers" to the cluster and appear consistent with the predictions of the closed box model. This fact agree with the scenario that these galaxies experience a pressure-triggered starburst, right before 
the ram-pressure stripping privies them from their gas component. The rest of the galaxies with HI measurement on average show lower values of gas fractions, though most of them are still consistent with the loci defined by samples of field galaxies.

5) The properties of the underlying stellar population, such as stellar age and stellar metallicity, have been explored and compared with the gas-phase metallicity. The "newcomers" dwarfs present a young stellar population and their stellar and gas metallicities are similar. The more metallic dwarf galaxies host an old stellar population, resembling to the evolved blue dwarfs refered by Mahajan et al. (2011). An overall positive trend has been found in the gas-phase oxygen abundance versus the mass-weighted stellar age $\tau_{\star, M}$, which becomes more prominent in the case of the $\mathrm{N} / \mathrm{O}$ ratio.

We have learned that the local environment of a galaxy is one of the key parameters in order to understand its chemical history. In the variety of the Hercules cluster ecosystem we have seen galaxygalaxy interactions, galaxy ISM-ICM interactions and candidates of tidal dwarfs galaxies. Further observations are needed to disentangle the role of all these environmental effects from the expected intrinsic galaxy evolution.

V.P. would like to thank Enrique Perez Montero, Ricardo Amorin, Ovidiu Vaduvescu, Ana Monreal-Ibero and Josep Maria Solanes for their help and suggestions. We thank the anonymous referee for the useful suggestions which helped to improve the paper. V.P also thanks the Osservatorio di Arcetri, the Centro de Astrofísica de La Palma and the Department of Astronomy in the University of Athens for hospitality during the stays to carry out part of this work. Special thanks are owed to M. Kontizas and E. Kontizas for their support.

V.P. acknowledge financial support from the Spanish Ministerio de Ciencia e Innovación under grant FPU AP2006-04622. We also acknowledge financial support by the Spanish PNAYA, projects ESTALLIDOS (grant AYA2007-67965C03-02) and CSD2006-00070 1st Science with GTC from the CONSOLIDER 2010 programme of the Spanish MICINN. We thank the Spanish CSIC for financial support (Proyecto Intramural 200850I018). P.P. is supported by a Ciencia 2008 contract, funded by FCT/MCTES (Portugal) and $\mathrm{POPH} / \mathrm{FSE}$ (EC).

The INT and WHT are operated on the island of La Palma by the Isaac Newton Group in the Spanish Observatorio del Roque de los Muchachos of the Instituto de Astrofísica de Canarias. We acknowledge CAT for the allocation of telescope time to this project. We thank the support astronomers of WHT (ING) for Service Time observations on June 26-27, 2009 and the directors of IAC and ING for the allocation of DDT on July 19, 2009.

This research has made use of the NASA/IPAC Extragalactic Database (NED), which is operated by the Jet Propulsion Laboratory, California Institute of Technology, under contract with the National Aeronautics and Space Administration. We also have benefitted from Sloan Digital Sky Survey (SDSS) database. Funding for the SDSS and SDSS-II was provided by the Alfred P. Sloan Foundation, the Participating Institutions, the National Science Foundation, the U.S. Department of Energy, the National Aeronautics and Space Administration, the Japanese Monbukagakusho, the Max Planck Society, and the Higher Education Funding Council for England. The SDSS was managed by the Astrophysical Research Consortium for the Participating Institutions. IRAF is distributed by the National Optical Astronomical Observatory, which is operated by the Associated Universities for Research in Astronomy, Inc., under cooperative agreement with the National Science Foundation.

Facilities: ING:Newton, ING:Herschel. 


\section{A. NOTES ON INDIVIDUAL GALAXIES}

\section{A.1. Interacting galaxies}

\section{IC1182, IC1182:[S72]d and SDSS J160531.84+174826.1:}

IC1182 is a late-merger that undergoes vigorous nuclear starburst activity with a star formation rate, estimated from its infrared and extinction-corrected $\mathrm{H} \alpha$ luminosity, to be between 11 and $\sim 90 \mathrm{M}_{\odot} / \mathrm{yr}$ (Radovich et al. 2005; Moles et al. 2004). IC1182 shows an extended ( 60 kpc) stellar tail to the east, where a tidal dwarf candidate IC1182:[S72]d is discernible (identified by Iglesias-Páramo et al. (2003); named ce-061 after Dickey (1997)). To the north of the system and to a comparable distance there are also distinguished faint tidal features and plumes. In addition, to the west of the merging system, at a projected distance of $\sim 60 \mathrm{kpc}$, there is the dwarf galaxy SDSS J160531.84+174826.1 which has been claimed (Dong et al. 2007) to be a Seyfert 1 AGN (but see 44). Dickey (1997) reports a long HI structure extending northwest of IC1182, creating a bridge of HI clumps towards SDSSJ150531.84+174826.1 (see Fig. 3.a of IP03), although the galaxy SDSSJ150531.84+174826.1 itself was not detected as a consequence of the velocity cuttoff $(9820 \mathrm{~km} / \mathrm{s})$ of the spectrometer used by Dickev (1997). These lines of evidence point towards a physical connection among the three galaxies. A forthcoming work will discuss the global picture of this system (Petropoulou et al., in prep).

\section{NGC6050:}

It has long been considered as a collision between two spiral galaxies (NGC6050A and NGC6050B). From our long-slit spectrum we found that these two galaxies show important velocity difference $\left(\sim 1600 \mathrm{~km} \mathrm{~s}^{-1}\right)$ suggesting that they might be just a chance galaxy alignment. On the other hand, to the north of the system we have identified another galaxy, on the basis of its underlying stellar component (clearly seen in the 2MASS imaging) and its SDSS spectrum. This galaxy is probably in interaction with NGC6050A. Dickey (1997) quotes an HI mass for the NGC6050 system. We attributed the HI mass exclusively to NGC6050B because NGC6050A is out of the velocity cutoff of the spectrometer used by Dickev (1997). The HI distribution is centered on NGC6050B.

\section{NGC6045:}

This edge-on spiral could be interacting with a smaller spiral companion located at the east of it. On its H $\alpha$ map (C09) we can see intense star-burst activity on the east part of the galaxy disk, probably triggered by this interaction. Huang \& Sarazin (1996) suggested a possible interaction of NGC6045 with the radio galaxy NGC6047 which is about $1^{\prime} .6(\sim 74 \mathrm{kpc})$ to the south. Dickey (1997) reports that in HI only half of the disk is visible. From our long-slit spectroscopy we find a large large velocity gradient along the disk of the galaxy and half of the disk shows velocity out of the cut-off of the spectrometer used by Dickev (1997).

\section{PGC057064:}

This peculiar galaxy, until now considered as one galaxy, has turned out to be a merger of two galaxies, which on our long-slit spectrum show different rotation velocities. We have considered the corresponding 2D spectrum parts separately. The 1D spectrum part (a) corresponds to the NW galaxy and (b) to the SE. The two dwarf galaxies comprising this merger do not show any significant chemical variation.

\section{A.2. Peculiar galaxies}

PGC057077: This is a peculiar galaxy previously cataloged as an elliptical in LEDA. The C09 $\mathrm{H} \alpha$ survey showed that PGC057077 is a very intense and compact $\mathrm{H} \alpha$ emitter with $f_{H_{\alpha}}=87 \times 10^{-15} \mathrm{erg} \mathrm{cm}^{-2} \mathrm{~s}^{-1}$ and $E W=148 \AA$. Although the galaxy is very compact and does not show any structural properties, we noted on its $g-i$ map, illustrated in Fig. 18, a conspicuous color gradient. In our long-slit spectrum we were able to identify two regions with important difference of the continuum emission. In order to check for velocity gradient and differences in the chemical content between these two regions, we separated the 2D spectrum in two corresponding 1D spectra. The important continuum emission difference of the 1D spectra can be contemplated in Fig. 17. From our emission-line analysis we do not find any significant velocity variation between the two different galaxy parts, neither any difference in the chemical abundances. The difference in 
the continuum emission is attributed then to the high extinction suffered by both the stellar population and the gas in the NW part of the galaxy which correspond to our (a) spectrum part. This peculiar galaxy is situated close to the edge of the principal X-Ray emitting region, at $650 \mathrm{kpc}$ from the X-Ray center. In the $\mathrm{NE}$ of the galaxy there is discernible a low surface blue plume, that could point toward the existence of a faint companion. The existing data are not conclusive whether it could have been the ICM-ISM interaction or a galaxy merger that could have triggered the unusual starburst of this galaxy. Further mid-infrared observations would be of interest there.

SDSSJ160520.58+175210.6 appears to be embedded into the HI structure assigned to the galaxy [D97]ce200 (SDSSJ160520.64+ 175201.5); Dickey (1997) reports that this system looks like the Magellanic Clouds and the Magellanic Stream. However these two galaxies have a velocity difference of $1300 \mathrm{~km} / \mathrm{s}$ a fact that renders unlikely an actual interaction of them. The velocity of the HI cloud is very similar to the velocity of the galaxy [D97]ce-200.

LEDA084703: This is one of the two galaxies of this sample situated in the substructure almost 1 Abell radius SW from the cluster center. It is a quite blue galaxy, classified Sd/Irr and it is situated almost one Abell radius away from the cluster center. There has been a SN explosion reported by Zwicky et al. (1969) in the East part of this irregular galaxy. There is a long plume extending towards southeast detected in the optical images as well as in HI by Dickey (1997). The west part of the disk hosts a very intense star-burst activity as can be seen in its $\mathrm{H} \alpha$ EW map in Fig. 18

SDSSJ160524.27+175329.3: It has typical $\mathrm{H} \alpha$ morphology and colors of a blue compact galaxy. This compact starburst, located close to the X-ray maximum in the center of the Hercules cluster could have resulted from the compression of the interstellar gas of a dwarf galaxy when entering the cluster core. Similar compact starburst galaxies have been found in the cores of other nearby clusters by Reverte et al. (2007).

SDSS J160556.98+174304.1 The $\mathrm{H} \alpha$ map of this dwarf galaxy shows an off-center starburst. Additionally this galaxy is located close to the edge of the primary X-ray maximum in the cluster center and presents a large velocity difference with NGC6041A. These evidences could suggest that the starburst has been triggered by the effect of the hot ICM. (Dickev 1997) have not detected molecular gas for this galaxy, suggesting that the ram-pressure stripping could have already taken off its molecular gas.

LEDA084724 presents an extended knotty $\mathrm{H} \alpha$ structure NW, in the direction towards the X-ray cluster center. This morphology could be suggestive of a starburst triggered by the ICM. (Dickev 1997) have not detected molecular gas for this galaxy.

SDSSJ160304.20+171126.7 is one of the two SF galaxies of this spectroscopic sample situated in the substructure almost 1 Abell radius SW from the cluster center. This galaxy has a close companion, the galaxy SDSS J160305.24+171136.1. The disturbed $\mathrm{H} \alpha$ structure of both could suggest a possible interaction.

SDSSJ160523.66+174832.3 is located very close to the faint galaxy SDSSJ160523.67+174828.8 (\#22 and \#23 in C09). The $\mathrm{H} \alpha$ map as well as the $g-i$ color map of SDSSJ160523.66+174832.3 seem to reveal a disturbed morphology. There could be the possibility that the two galaxies are interacting, although SDSSJ $160523.67+174828.8$ does not have spectroscopic data and SDSS provide a photo-z estimate of $z=0.15 \pm 0.02$, nominally not consistent with the velocity range of Hercules cluster.

[DKP87]160310.21+175956.7 is projected close to a faint galaxy located southern-east. The H $\alpha$ EW map of [DKP87]160310.21+175956.7 shows an intense star-burst event towards that direction. However the close companion galaxy has no spectroscopic data and the photo-z estimate provided by SDSS $z=0.25 \pm 0.06$ is not consistent with the velocity range of Hercules cluster.

SDSSJ160547.17+173501.6: It has turned out to be a background galaxy with $z=0.12 \pm 0.01$. The various SDSS photo-z estimates were $0.06 \pm 0.01,0.06 \pm 0.05$ and $0.10 \pm 0.04$, thus the criteria adopted by C09 for cluster membership were fulfilled. 


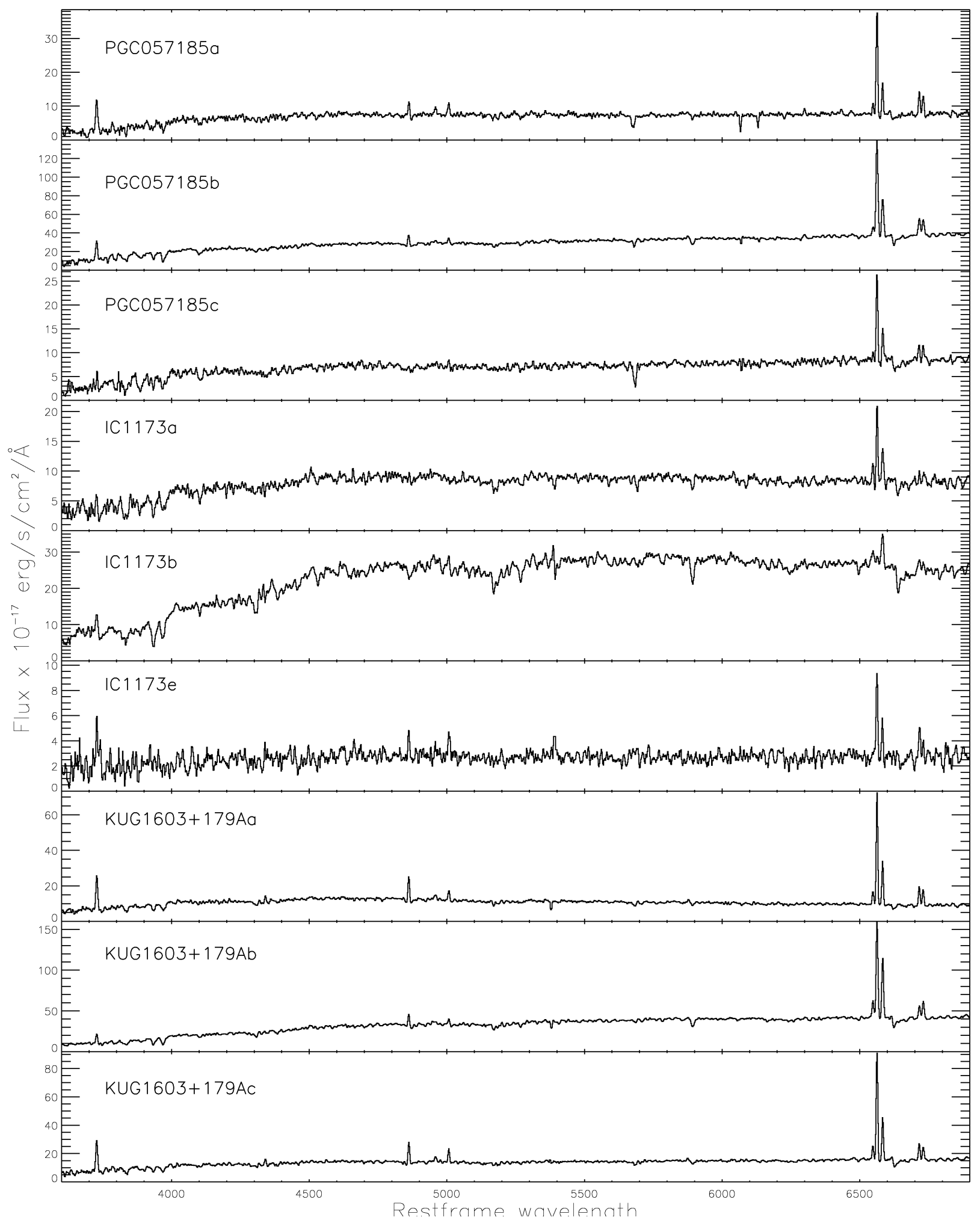

Fig. 17.- Integrated 1D observed spectra for each galaxy or part of a galaxy by INT and WHT. 


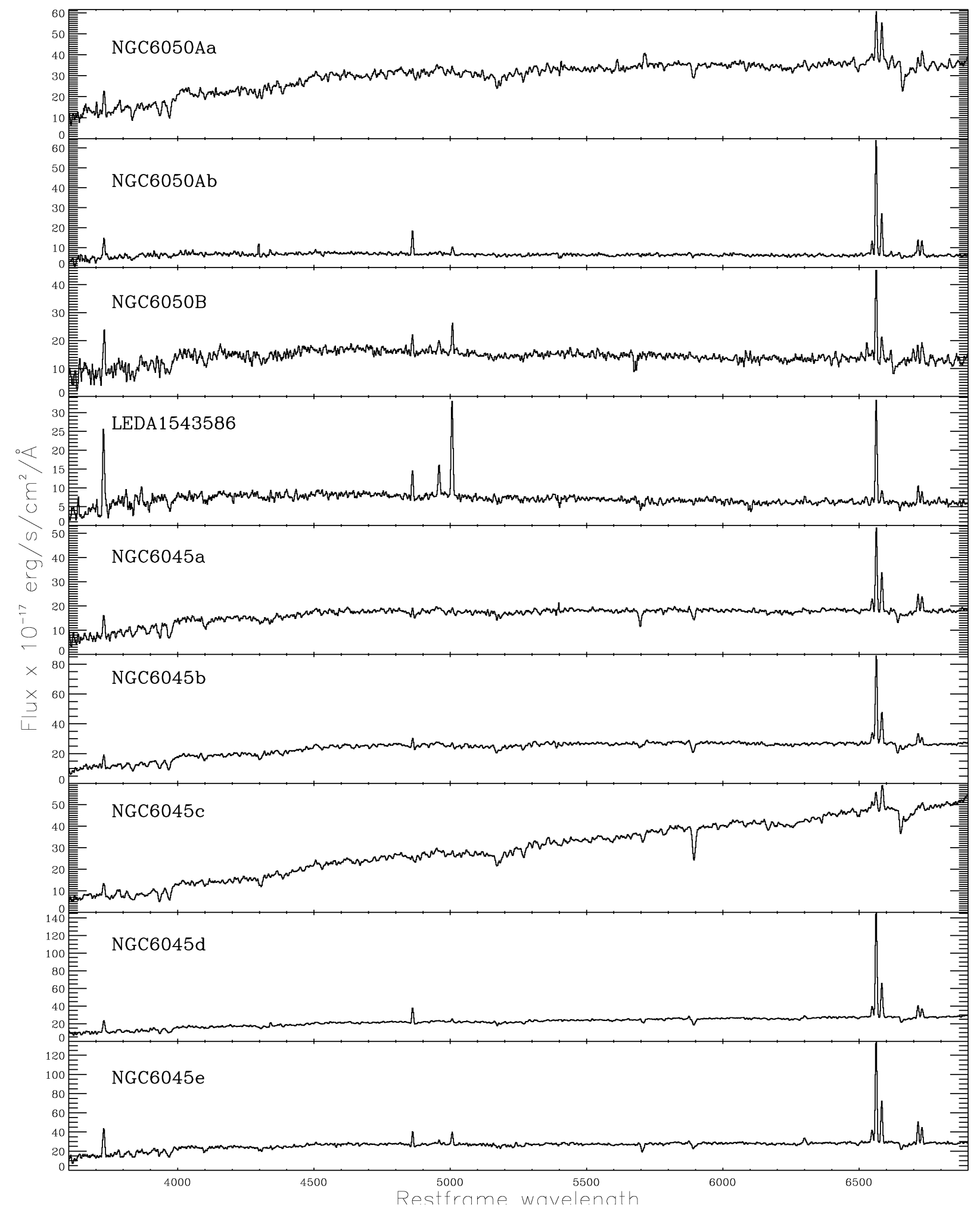

Fig. 17.- Continued. 


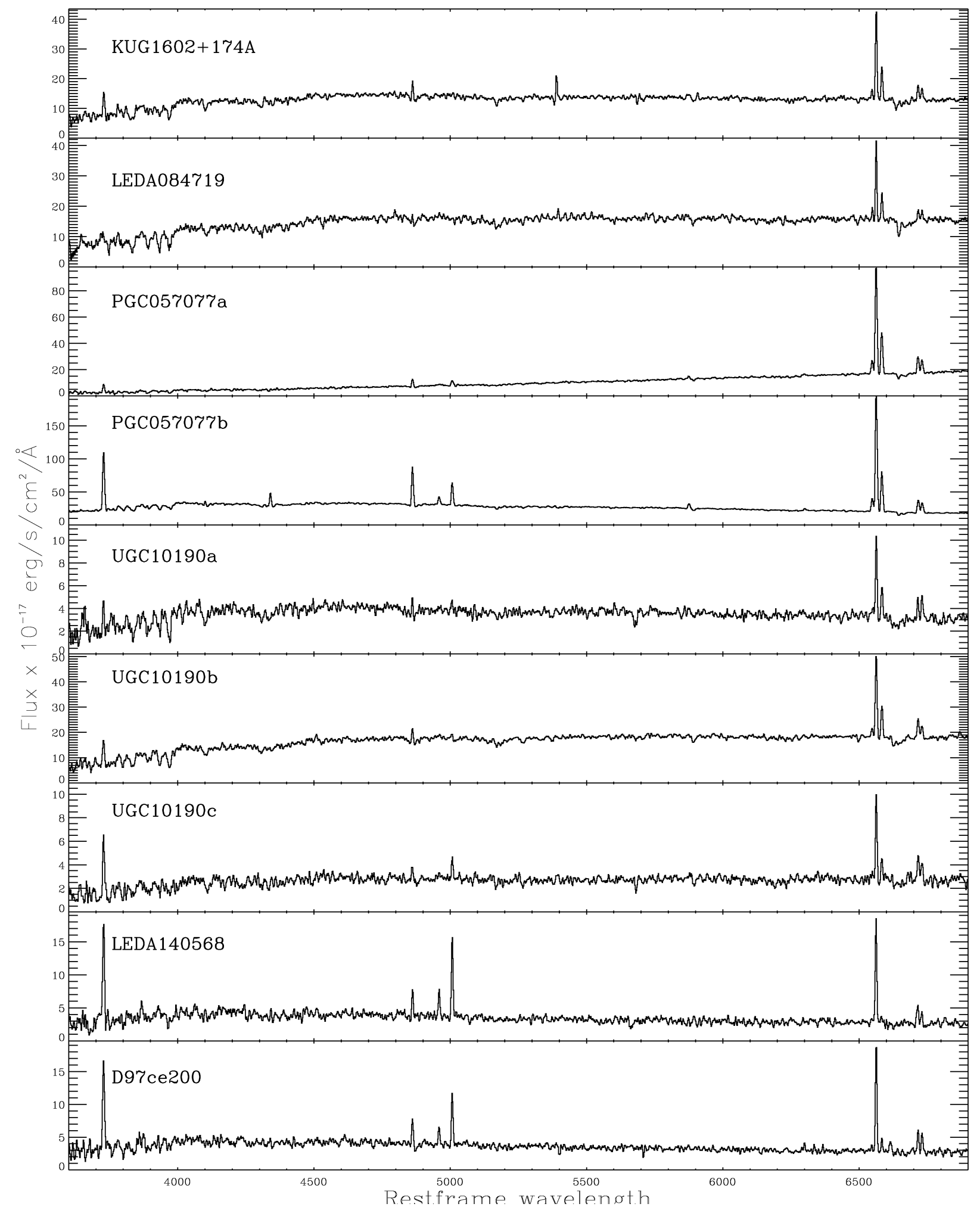

Fig. 17.- Continued. 


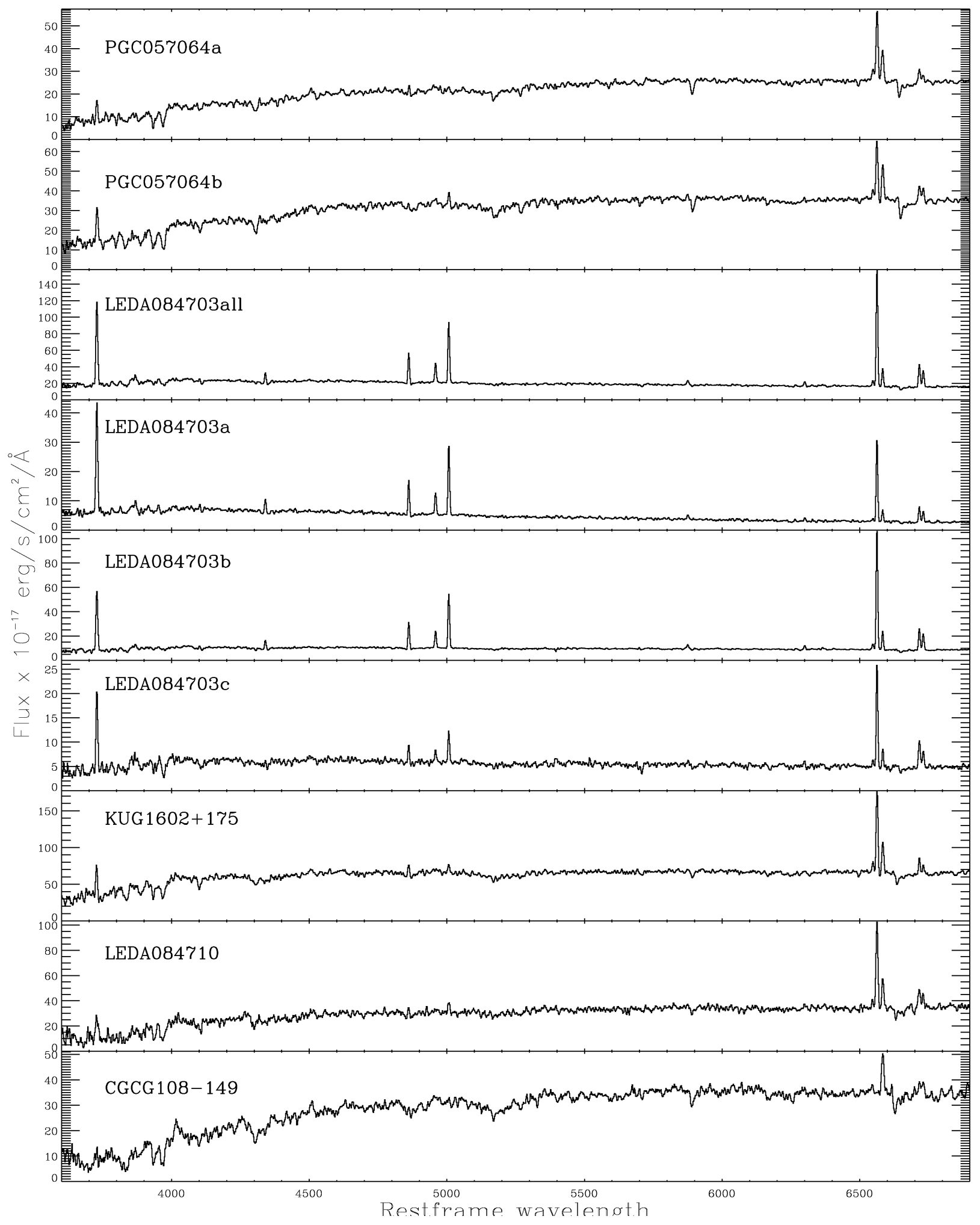

Fig. 17.- Continued. 


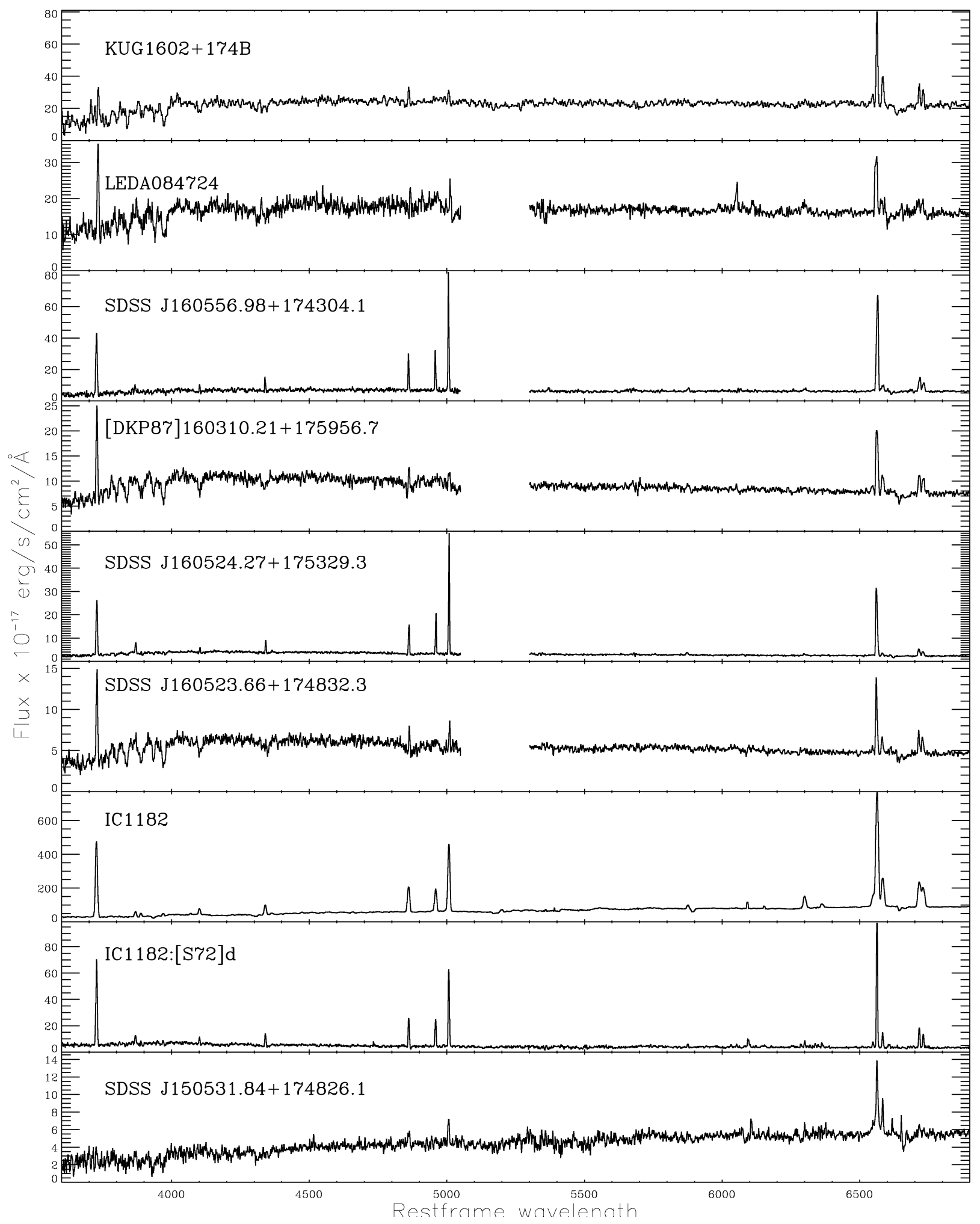

Fig. 17.- Continued. 

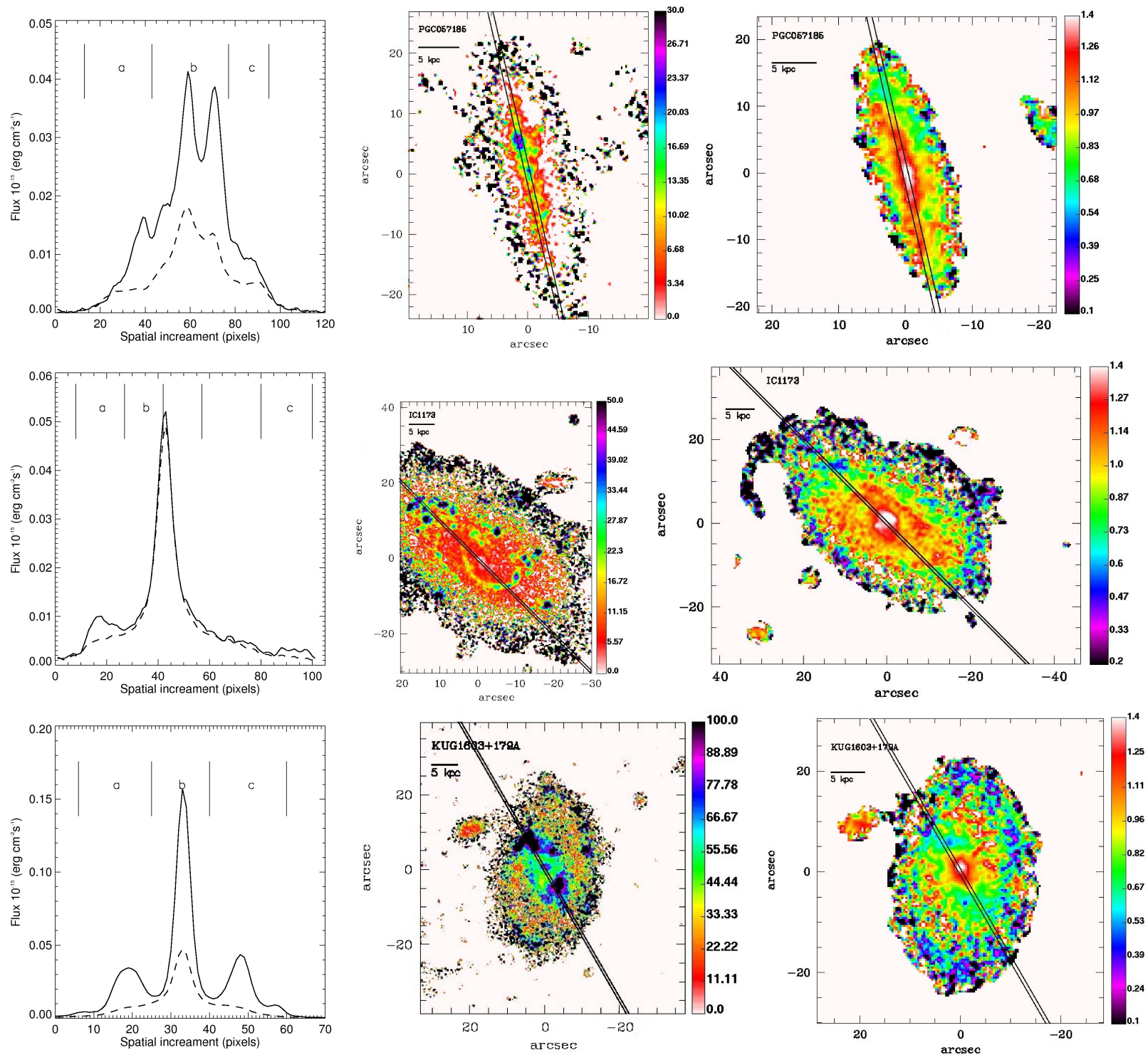

Fig. 18. - The first column shows the spatial profiles of the $\mathrm{H} \alpha$ line (continuous) and nearby continuum (dashed line) along the slit extracted from the 2D spectra, for the galaxies showing rich spatial structure. The different sub-regions used to divide their 2D spectra into different 1D spectra are shown. The second and third column show respectively the color ( $\left.\mathrm{g}^{\prime}-\mathrm{i}^{\prime}\right)$ maps and the $\mathrm{H} \alpha \mathrm{EW}$ maps, with the slit position overploted. 

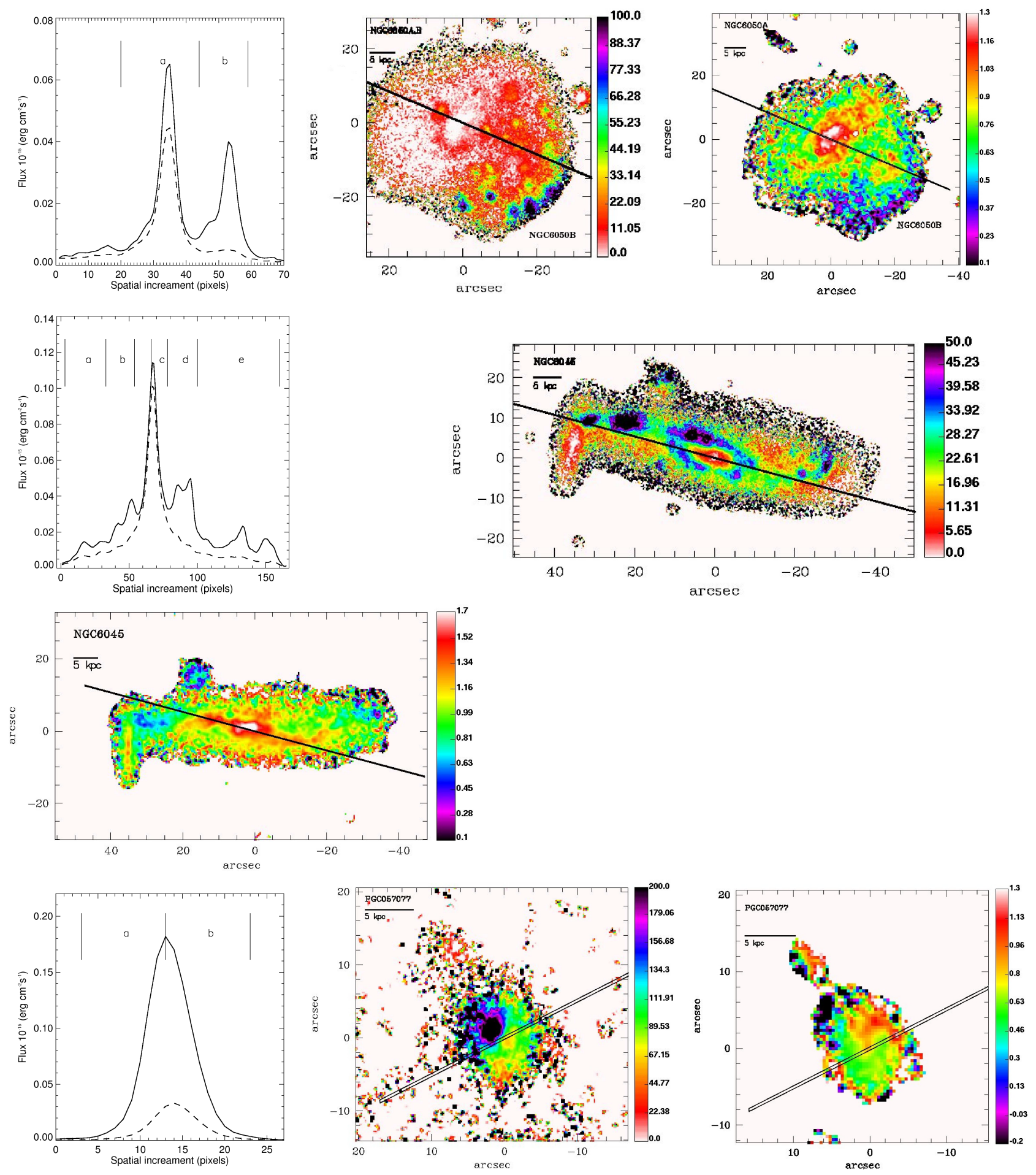

Fig. 18.- Continued. 

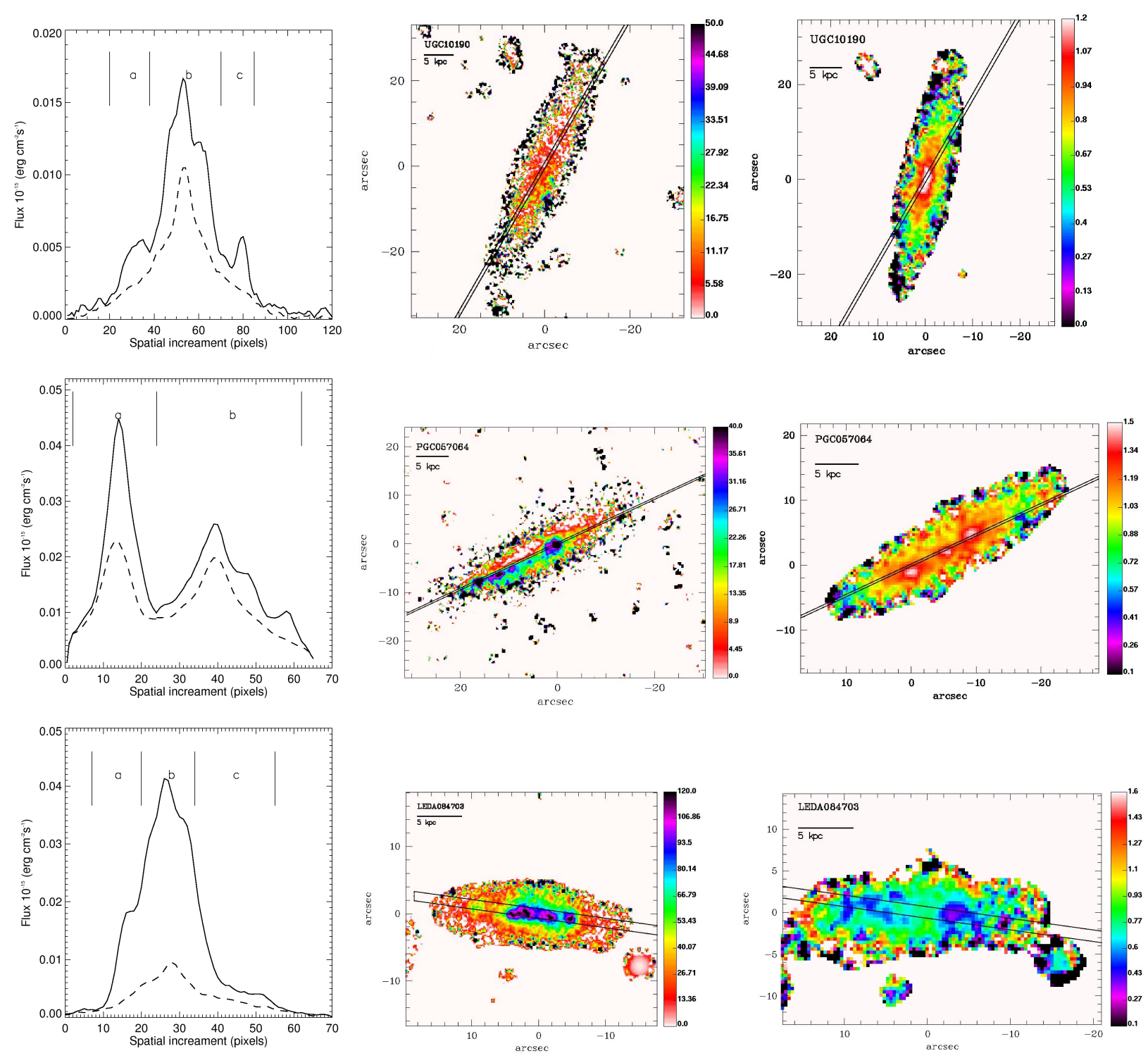

Fig. 18.- Continued. 


\section{REFERENCES}

Abadi, M. G., Moore, B., \& Bower, R. G. 1999, MNRAS, 308, 947

Alonso-Herrero, A., Garcia-Marin, M., Rodriguez Zaurin, J., Monreal-Ibero, A., Colina, L., \& Arribas, S. 2010, arXiv:1006.2219

Amorín, R. O., Pérez-Montero, E., \& Vílchez, J. M. 2010, ApJ, 715, L128

Asari, N.V., Cid Fernandez, R., Stasinska, G. et al. 2007, MNRAS, 381, 263

Asplund, M., Grevesse, N., Sauval, A. J., \& Scott, P. 2009, ARA\&A, 47, 481

Baldwin, J. A., Phillips, M. M., \& Terlevich, R. 1981, PASP, 93, 5

Balogh, M., et al. 2004, MNRAS, 348, 1355

Barmby, P., \& Huchra, J. P. 1998, AJ, 115, 6

Barnes, J. E., \& Hernquist, L. 1996, ApJ, 471, 115

Bell, E. F., McIntosh, D. H., Katz, N., \& Weinberg, M. D. 2003, ApJS, 149, 289

Bird, C. M., Davis, D. S., \& Beers, T. C. 1995, AJ, 109, 920

Blanton, M. R., \& Roweis, S. 2007, AJ, 133, 734

Boselli, A., \& Gavazzi, G. 2006, PASP, 118, 517

Bresolin, F., Gieren, W., Kudritzki, R.-P., Pietrzyński, G., Urbaneja, M. A., \& Carraro, G. 2009, ApJ, 700, 309

Bretherton, C. F., James, P. A., Moss, C., \& Whittle, M. 2010, A\&A, 524, A24

Bruzual, G., \& Charlot, S. 2003, MNRAS, 344, 1000

Calura, F., Pipino, A., Chiappini, C., Matteucci, F., \& Maiolino, R. 2009, A\&A, 504, 373

Calzetti, D., Kinney, A. L., \& Storchi-Bergmann, T. 1994, ApJ, 429, 582

Cardelli, J. A., Clayton, G. C., \& Mathis, J. S. 1989, ApJ, 345, 245
Cedrés, B., Iglesias-Páramo, J., Vílchez, J. M., Reverte, D., Petropoulou, V., \& HernándezFernández, J. 2009, AJ, 138, 873

Chabrier, G. 2003, PASP, 115, 763

Cid Fernandes, R., Mateus, A., Sodré, L., Stasińska, G., \& Gomes, J. M. 2005, MNRAS, 358,363

Cid Fernandes, R., González Delgado, R. M., Storchi-Bergmann, T., Martins, L. P., \& Schmitt, H. 2005, MNRAS, 356, 270

Cid Fernandes, R., Gu, Q., Melnick, J., Terlevich, E., Terlevich, R., Kunth, D., Rodrigues Lacerda, R., \& Joguet, B. 2004, MNRAS, 355, 273

Conselice, C. J. 2006, ApJ, 638, 686

Cooper, M. C., Tremonti, C. A., Newman, J. A., \& Zabludoff, A. I. 2008, MNRAS, 390, 245

Cortese, L., Gavazzi, G., Boselli, A., Franzetti, P., Kennicutt, R. C., O’Neil, K., \& Sakai, S. 2006, A\&A, 453, 847

Davé, R., Finlator, K., Oppenheimer, B. D., Fardal, M., Katz, N., Kereš, D., \& Weinberg, D. H. 2010, MNRAS, 404, 1355

Dickey, J. M. 1997, AJ, 113, 1939

Dong, X., et al. 2007, ApJ, 657, 700

Dressler, A. 1980, ApJ, 236, 351

Drory, N., Bender, R., \& Hopp, U. 2004, ApJ, 616, L103

Duc, P.-A., Braine, J., Lisenfeld, U., Brinks, E., \& Boquien, M. 2007, A\&A, 475, 187

Duc, P.-A., Cayatte, V., Balkowski, C., Thuan, T. X., Papaderos, P., \& van Driel, W. 2001, A\&A, 369, 763

Duc, P.-A., Papaderos, P., Balkowski, C., Cayatte, V., Thuan, T. X., \& van Driel, W. 1999, A\&AS, 136,539

Edmunds, M. G. 1990, MNRAS, 246, 678

Ellison, S. L., Simard, L., Cowan, N. B., Baldry, I. K., Patton, D. R., \& McConnachie, A. W. 2009, MNRAS, 396, 1257 
Erb, D. K., Shapley, A. E., Pettini, M., Steidel, C. C., Reddy, N. A., \& Adelberger, K. L. 2006, ApJ, 644, 813

Ferrini, F., Molla, M., Pardi, M. C., \& Diaz, A. I. 1994, ApJ, 427, 745

Finlator, K., \& Davé, R. 2008, MNRAS, 385, 2181

Finn, R. A., et al. 2005, ApJ, 630, 206

Fukugita, M., Shimasaku, K., \& Ichikawa, T. 1995, PASP, 107, 945

Gallazzi, A., Charlot, S., Brinchmann, J., White, S. D. M., \& Tremonti, C. A. 2005, MNRAS, 362,41

Garcia-Rissmann, A., Vega, L. R., Asari, N. V., Cid Fernandes, R., Schmitt, H., González Delgado, R. M., \& Storchi-Bergmann, T. 2005, MNRAS, 359, 765

Gavazzi, G., Boselli, A., Mayer, L., IglesiasParamo, J., Vílchez, J. M., \& Carrasco, L. 2001, ApJ, 563, L23

Giovanelli, R., Haynes, M. P., \& Chincarini, G. L. 1981, ApJ, 247, 383

González Delgado, R.M., Leitherer, C., Heckman, T.M. 1999, ApJS, 125, 489

Gunn, J. E., \& Gott, J. R., III 1972, ApJ, 176, 1

Guseva, N.G., Izotov, Y.I., Papaderos, P., Chaffee, C.B., Foltz, C.B., Green, R.F., Fricke, K.J., Noeske, K.G. 2001, A\&A, 378, 756

Haines, C. P., Gargiulo, A., La Barbera, F., Mercurio, A., Merluzzi, P., \& Busarello, G. 2007, MNRAS, 381, 7

Hägele, G. F., Díaz, Á. I., Terlevich, E., Terlevich, R., Pérez-Montero, E., \& Cardaci, M. V. 2008, MNRAS, 383, 209

Henry, R. B. C., Balkowski, C., Cayatte, V., Edmunds, M. G., \& Pagel, B. E. J. 1996, MNRAS, 283,635

Henry, R. B. C., Pagel, B. E. J., \& Chincarini, G. L. 1994, MNRAS, 266, 421

Henry, R. B. C., Pagel, B. E. J., Lasseter, D. F., \& Chincarini, G. L. 1992, MNRAS, 258, 321
Huang, Z., \& Sarazin, C. L. 1996, ApJ, 461, 622

Iglesias-Páramo, J., et al. 2003, A\&A, 406, 453

Jabran Zahid, H., Kewley, L. J., \& Bresolin, F. 2010, arXiv:1006.4877

Jansen, R. A., Fabricant, D., Franx, M., \& Caldwell, N. 2000, ApJS, 126, 331

Kauffmann, G., et al. 2003, MNRAS, 346, 1055

Kenney, J. D. P., van Gorkom, J. H., \& Vollmer, B. 2004, AJ, 127, 3361

Kennicutt, R. C., Jr. 1998, ApJ, 498, 541

Kewley, L. J., Dopita, M. A., Sutherland, R. S., Heisler, C. A., \& Trevena, J. 2001, ApJ, 556, 121

Kewley, L. J., \& Dopita, M. A. 2002, ApJS, 142, 35

Kewley, L. J., \& Ellison, S. L. 2008, ApJ, 681, 1183

King, D. 1985, La Palma Teck. Note 31

Kobulnicky, H. A.,

Koopmann, R. A., \& Kenney, J. D. P. 2004, ApJ, 613,866

Köppen, J., Weidner, C., \& Kroupa, P. 2007, MNRAS, 375, 673

Lamareille, F., Mouhcine, M., Contini, T., Lewis, I., \& Maddox, S. 2004, MNRAS, 350, 396

Lara-López, M. A., et al. 2010, arXiv:1005.0509

Larson, R. B., Tinsley, B. M., \& Caldwell, C. N. 1980, ApJ, 237, 692

Lee, H., Zucker, D. B., \& Grebel, E. K. atteucci, F., \& Recchi, S. 2010, A\&A, 514, A73

Lee, H., McCall, M. L., \& Richer, M. G. 2003, AJ, 125,2975

Lequeux, J., Peimbert, M., Rayo, J. F., Serrano, A., \& Torres-Peimbert, S. 1979, A\&A, 80, 155

Li, C., \& White, S. D. M. 2009, MNRAS, 398, 2177 
Lopez-Sanchez, A. R., \& Esteban, C. 2010, arXiv:1004.0626

Lopez-Sanchez, A. R., \& Esteban, C. 2010, arXiv:1004.0626

Lopez-Sanchez, A. R. 2010, arXiv:1005.0659

Maccagni, D., Garilli, B., \& Tarenghi, M. 1995, AJ, 109, 465

Mahajan, S., Haines, C. P., \& Raychaudhury, S. 2010, MNRAS, 404, 1745

Mahajan, S., Haines, C. P., \& Raychaudhury, S. 2011, MNRAS, 7

Maiolino, R., et al. 2008, A\&A, 488, 463

Mamon, G. 1996, Third Paris Cosmology Colloquium , 95

Mannucci, F., Cresci, G., Maiolino, R., Marconi, A., \& Gnerucci, A. 2010, MNRAS, 1314

Mateus, A., Sodré, L., Cid Fernandes, R., \& Stasińska, G. 2007, MNRAS, 374, 1457

Meynet, G., \& Maeder, A. 2002, A\&A, 390, 561

Miller, J. S., \& Mathews, W. G. 1972, ApJ, 172, 593

McGaugh, S. S. 1991, ApJ, 380, 140

Mihos, J. C. 2004, Clusters of Galaxies: Probes of Cosmological Structure and Galaxy Evolution, 277

Moles, M., Bettoni, D., Fasano, G., Kjærgaard, P., Varela, J., \& Milvang-Jensen, B. 2004, A\&A, 418,495

Mollá, M., Vílchez, J. M., Gavilán, M., \& Díaz, A. I. 2006, MNRAS, 372, 1069

Monreal-Ibero, A., Vilchez, J. M., Walsh, J. R., \& Munoz-Tunon, C. 2010, arXiv:1003.5329

Moore, B., Lake, G., \& Katz, N. 1998, ApJ, 495, 139

Moore, B., Lake, G., Quinn, T., \& Stadel, J. 1999, MNRAS, 304, 465

Mouhcine, M., Baldry, I. K., \& Bamford, S. P. 2007, MNRAS, 382, 801
Moustakas, J., \& Kennicutt, R. C., Jr. 2006, ApJ, 651,155

Mulchaey, J. S., Davis, D. S., Mushotzky, R. F., \& Burstein, D. 1993, ApJ, 404, L9

Nagao, T., Maiolino, R., \& Marconi, A. 2006, A\&A, 459, 85

Pérez-Montero, E., \& Contini, T. 2009, MNRAS, 398, 949

Pettini, M., \& Pagel, B. E. J. 2004, MNRAS, 348, L59

Pilyugin, L. S., Mollá, M., Ferrini, F., \& Vílchez, J. M. 2002, A\&A, 383, 14

Pilyugin, L. S., \& Thuan, T. X. 2005, ApJ, 631, 231

Pilyugin, L. S., Vílchez, J. M., \& Thuan, T. X. 2010, ApJ, 720, 1738

Quilis, V., Moore, B., \& Bower, R. 2000, Science, 288,1617

Radovich, M., Ciroi, S., Contini, M., Rafanelli, P., Afanasiev, V. L., \& Dodonov, S. N. 2005, A\&A, 431,813

Reverte, D., Vílchez, J. M., Hernández-Fernández, J. D., \& Iglesias-Páramo, J. 2007, AJ, 133, 705

Reverte D. 2008, PhD thesis, Universidad de Granada

Sánchez-Janssen, R., Iglesias-Páramo, J., MuñozTuñón, C., Aguerri, J. A. L., \& Vílchez, J. M. 2005, A\&A, 434, 521

Savaglio, S., et al. 2005, ApJ, 635, 260

Shields, G. A., Skillman, E. D., \& Kennicutt, R. C., Jr. 1991, ApJ, 371, 82

Smith, R. J., Lucey, J. R., Hudson, M. J., Allanson, S. P., Bridges, T. J., Hornschemeier, A. E., Marzke, R. O., \& Miller, N. A. 2009, MNRAS, 392,1265

Skillman, E. D., Kennicutt, R. C., Jr., Shields, G. A., \& Zaritsky, D. 1996, ApJ, 462, 147

Skillman, E. D., Kennicutt, R. C., \& Hodge, P. W. 1989, ApJ, 347, 875 
Spitoni, E., Calura, F., Matteucci, F., \& Recchi, S. 2010, A\&A, 514, A73

Struck, C. 1999, Phys. Rep., 321, 1

Tremonti, C. A., et al. 2004, ApJ, 613, 898

Treu, T., Ellis, R. S., Kneib, J.-P., Dressler, A., Smail, I., Czoske, O., Oemler, A., \& Natarajan, P. 2003, ApJ, 591, 53

Vaduvescu, O., McCall, M. L., \& Richer, M. G. 2007, AJ, 134, 604

van Gorkom, J. H. 2004, Clusters of Galaxies: Probes of Cosmological Structure and Galaxy Evolution, 305

van Zee, L., \& Haynes, M. P. 2006, ApJ, 636, 214

Verheijen, M. A. W. 2004, IAU Colloq. 195: Outskirts of Galaxy Clusters: Intense Life in the Suburbs, 394

Vílchez, J. M., \& Iglesias-Páramo, J. 2003, ApJS, 145,225

Vilchez, J. M. 1995, AJ, 110, 1090

Vollmer, B., Cayatte, V., Balkowski, C., \& Duschl, W. J. 2001, ApJ, 561, 708

Weisz, D. R., et al. 2011, arXiv:1101.1093

Yasuda, N., et al. 2001, AJ, 122, 1104

Zaritsky, D., Kennicutt, R. C., Jr., \& Huchra, J. P. 1994, ApJ, 420, 87

Zwicky, F., Sargent, W. L. W., \& Kowal, C. 1969, PASP, 81, 224

This 2-column preprint was prepared with the AAS LATEX macros v5.2. 